Aus der Klinik für Gastroenterologie und gastrointestinale Onkologie

(Prof. Dr. med. V. Ellenrieder)

der Medizinischen Fakultät der Universität Göttingen

\title{
Ischämische Cholangiopathie bei nicht lebertransplantierten Patienten - eine retrospektive Analyse bei Patienten der UMG
}

\author{
INAUGURAL-DISSERTATION \\ zur Erlangung des Doktorgrades \\ der Medizinischen Fakultät der \\ Georg-August-Universität zu Göttingen
}

vorgelegt von

Ardian Mekolli

aus Prishtina

Göttingen 2015 
Dekan:

1. Berichterstatter:

2. Berichterstatter/in:

3. Berichterstatter/in:
Prof. Dr. rer. nat. H. K. Kroemer

Prof. Dr. med. H. Schwörer

Tag der mündlichen Prüfung: 


\section{Inhaltsverzeichnis}

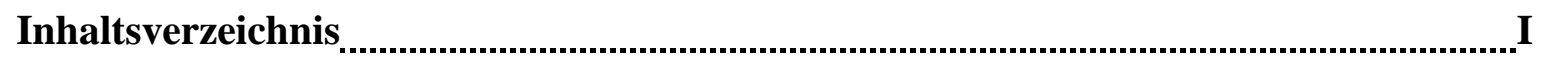

Abkürzungsverzeichnis ................................................................................................. III

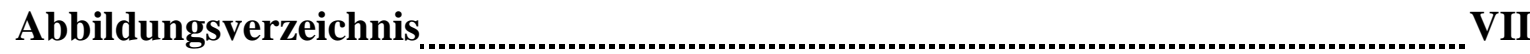

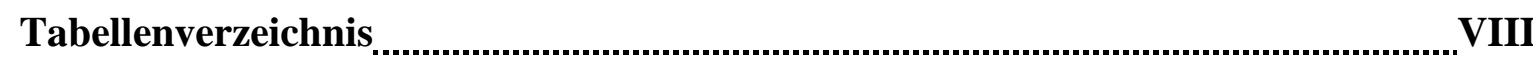

1 Einleitung

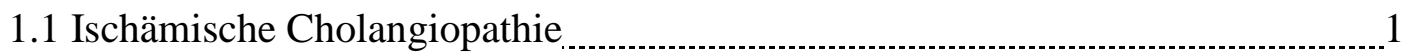

1.2 Ätiologie

1.3 Epidemiologie $\ldots \ldots \ldots \ldots \ldots \ldots \ldots \ldots+2$

1.4 Diagnostik _ _ _ _ _ _ _ _

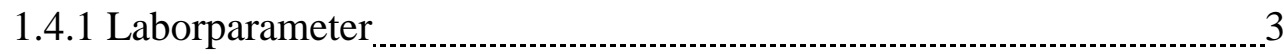

1.4.2 Bildgebende Verfahren …………………………………………........ 4

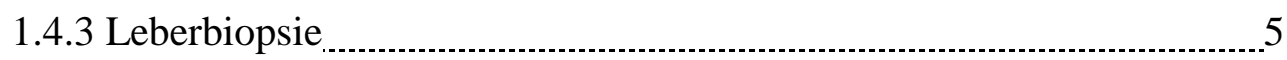

1.4.4 Endoskopische retrograde Cholangiographie (ERC) _......................... 5

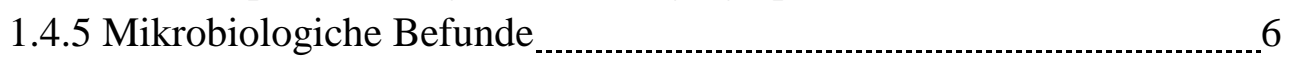

1.5 Therapie $\quad 6$

1.6 Prognose $\ldots \ldots$

2 Fragestellung

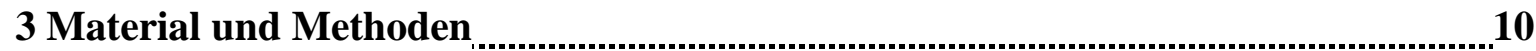

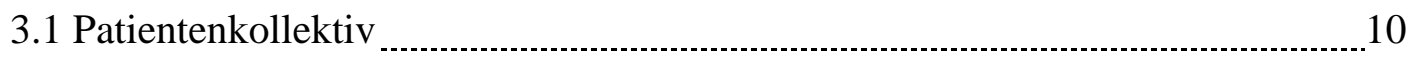

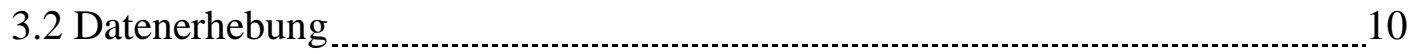

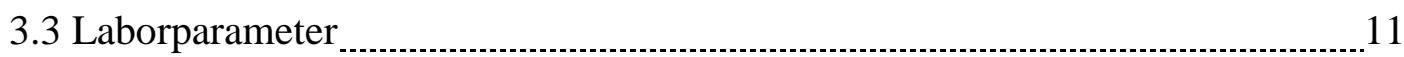

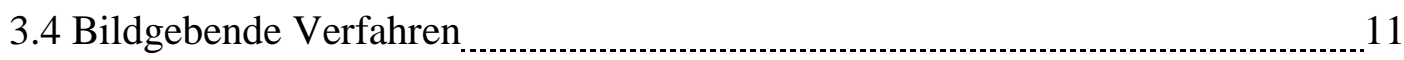

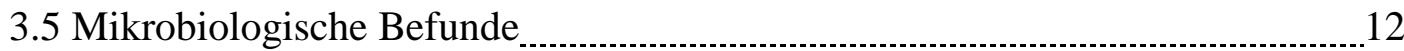

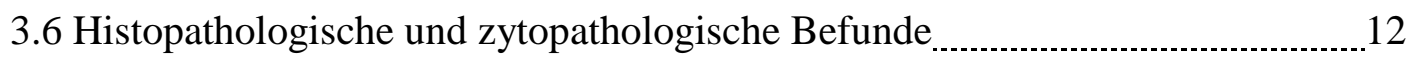

3.7 ERC-Befunde $\ldots \ldots$

4 Ergebnisse

4.1 Alter und Geschlechtsverteilung _............................................................ 13

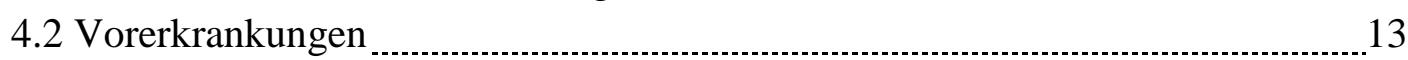

4.3 Anamnestische Risikofaktoren …………………………………………..... 15

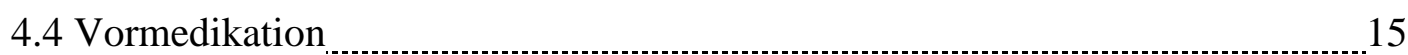

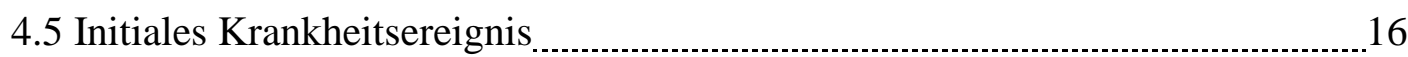

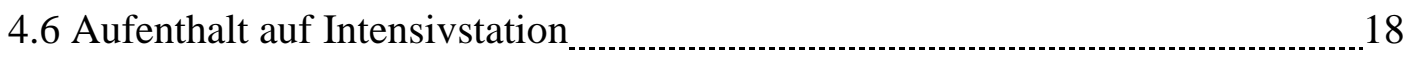

4.6.1 Invasive Maßnahmen während des Intensivaufenthaltes ......................19

4.6.2 Intensivmedikamentöse Therapie ......................................................2 20

4.6.2.1 Sedierung und Katecholamine während des Intensivaufenthaltes __................................ 20

4.6.2.2 Antiinfektiva während des Intensivaufenthaltes _................... 21 
4.6.2.3 Weitere Medikationen während des Intensivaufenthaltes _... 23 4.6.3 Laborparameter während des Intensivaufenthaltes _............................. 23

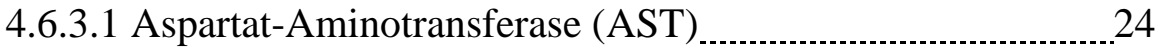

4.6.3.2 Alanin-Aminotransferase (ALT) _...…………........ 25

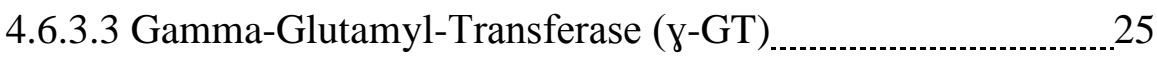

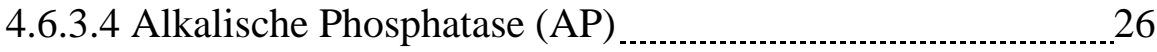

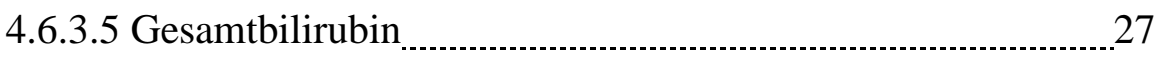

4.6.3.6 Quick-Wert _............................................................ 30

4.6.3.7 Kreatinin $\ldots$

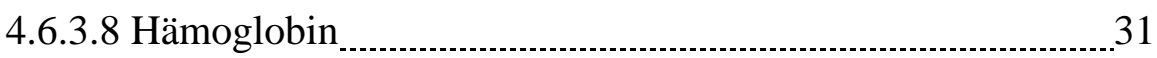

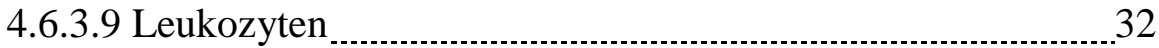

4.6.3.10 Arterielle Blutgasanalyse $\left(\mathrm{pH}, \mathrm{pO}_{2}, \mathrm{pCO}_{2}\right.$, Laktat) ............. 33

4.6.3.11 Laborwerte zum Zeitpunkt der Diagnosestellung mittels ERC ................................... 37

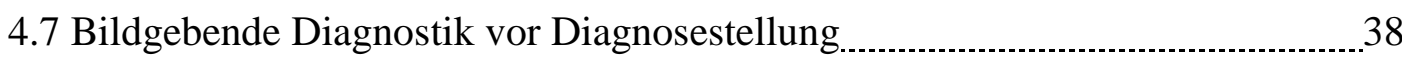

4.7.1 Abdomensonographie $\ldots$

4.7.2 Computertomographie (CT) _.................................................. 40

4.7.3 Endosonographie (EUS) _.................................................... 41

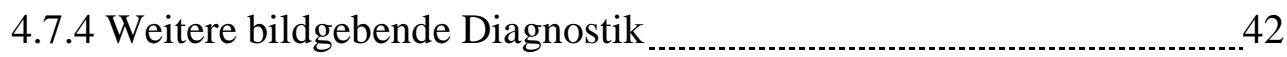

4.8 Leberbiopsie und histopathologische Diagnostik _................................... 42

4.9 Bakteriologische Befunde vor Diagnosesicherung _ $\quad 43$

4.9.1 Bakteriologische und mykologische Befunde im Galleaspirat _.......... 48

4.10 Diagnosesicherung mittels ERC _ _ $\quad 49$

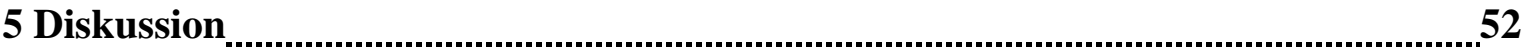

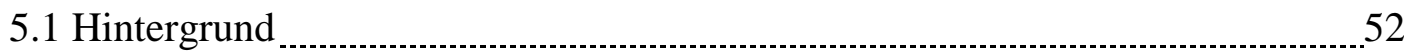

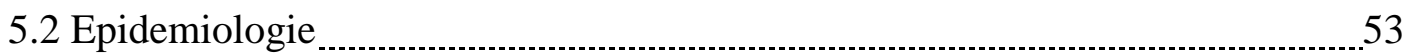

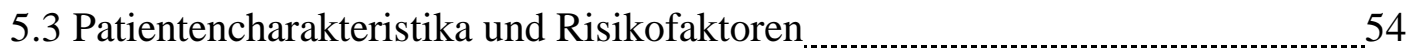

5.4 Intensivaufenthalt $\ldots \ldots \ldots$

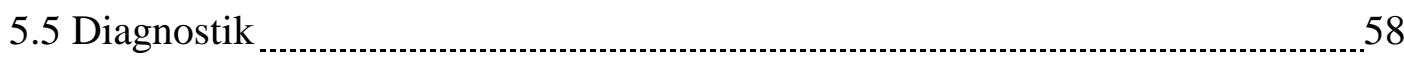

5.5.1 Laborparameter $\quad 58$

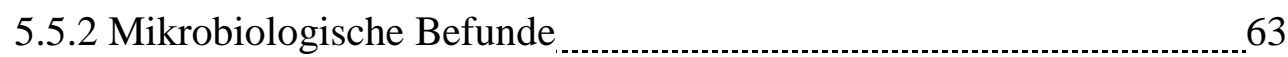

5.5.3 Bildgebende Diagnostik

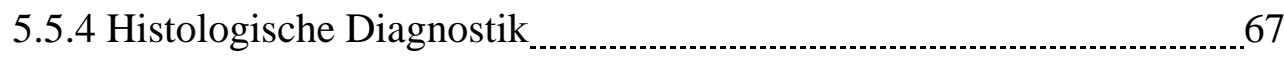

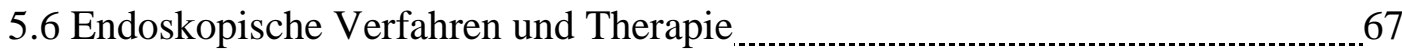

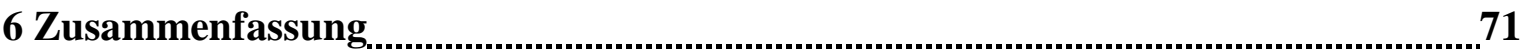

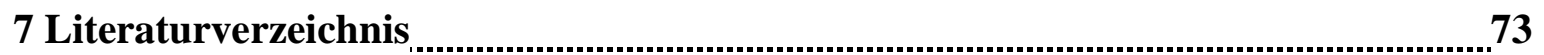




\section{Abkürzungsverzeichnis}

A

A.

ACE

ACS

ACVB

AIDS

ALT

AP

ARDS

AST

AT1

B

BCS

BGA

C

CED

CMV

COPD

CRP

CT

CVVH

$\mathbf{E}$

EBV

E.coli
Arteria

Angiotensin converting enzyme

Acute coronary syndrome, akutes Koronarsyndrom

Aortokoronarer Venenbypass

Acquired Immune Deficiency Syndrome

Alanin-Aminotransferase

Alkalische Phosphatase

Acute respiratory distress syndrome

Aspartat-Aminotransferase

Angiotensin 1

Biliary cast syndrome

Blutgasanalyse

Chronisch entzündliche Darmerkrankungen

Cytomegalievirus

Chronical obstructive pulmonary disease

C-reaktives Protein

Computertomographie

Continuous veno-venous hemofiltration

Epstein-Barr-Virus

Escherichia coli 
ECMO

EPT

ERC

ERCP

EUS

G

y-GT

ggf.

H

$\mathrm{Hb}$

HBV

$\mathrm{HCV}$

HSV

HFO

I

IABP

ICB

ICU

$\mathrm{IgG}_{4}$

ITBL

K

KHK

M

m

MRCP
Extrakorporale Membranoxigenierung

Endoskopische Papillotomie

Endoskopische retrograde Cholangiographie

Endoskopische retrograde

Cholangiopankreatikographie

Endoskopischer Ultraschall, Endosonographie

Gamma-Glutamyltransferase

gegebenenfalls

Hämoglobin

Hepatitis-B-Virus

Hepatitis-C-Virus

Herpes simplex-Virus

Hochfrequenzoszillations-Beatmung

Intraaortale Ballonpumpe

Intrakranielle Blutung

Intensive care unit

Immunglobulin der Klasse $\mathrm{G}_{4}$

Ischemic-type biliary lesions

Koronare Herzkrankheit

männlich

Magnetresonanz-Cholangiopankreatikographie 
MRT

\section{O}

o.g.

ORSA

ORSE

OSAS

OP

$\mathbf{P}$

pAVK

$\mathrm{pCO}_{2}$

$\mathrm{pO}_{2}$

PEG

PET

PT

PTT

PSC

$\mathbf{S}$

SC-CIP

SSC

Staph.

$\mathbf{T}$

TIA

$\mathbf{U}$

u.a

UDC
Magnetresonanztomographie, -tomogramm

oben genannt

Oxacillin-resistenter Staphylococcus aureus

Oxacillin-resistenter Staphylococcus epidermidis

Obstruktives Schlafapnoe-Syndrom

Operation

periphere arterielle Verschlusskrankheit

Kohlendioxidpartialdruck

Sauerstoffpartialdruck

Perkutane endoskopische Gastrostomie

Positronenemissionstomographie, -tomogramm

Prothrombinwert nach Quick, sog. Quick-Wert

Partielle Thromboplastinzeit

Primär sklerosierende Cholangitis

Secondary sclerosing cholangitis in critically ill patients

Sekundär sklerosierende Cholangitis

Staphylococcus

Transitorische Ischämische Attacke

unter anderem

Ursodesoxycholsäure 
UMG Universitätsmedizin Göttingen

V

V.

Vena

VRE

Vancomycin-resistenter Enterococcus

vs.

versus

VZV

Varizella zoster-Virus

W

w

weiblich

$\mathbf{Z}$

ZNS

Zentrales Nervensystem

ZVK

Zentraler Venenkatheter 


\section{Abbildungsverzeichnis}

Abbildung 1: Altersverteilung der Patienten mit ischämischer Cholangiopathie

Abbildung 2: Verteilung der Vorerkrankungen bei Patienten

Abbildung 3: Ereignisverteilung bei Patienten

Abbildung 4: Invasive Maßnahmen während des Intensivaufenthaltes

Abbildung 5: Anästhesie und Sedierung auf Intensivstation 21

Abbildung 6: Antibiotische/antimykotische/antivirale Therapie auf Intensivstation 23

Abbildung 7: Verlauf der AST-Medianwerte 24

Abbildung 8: Verlauf der ALT-Medianwerte 25

Abbildung 9: Verlauf der $\mathrm{\gamma}$-GT-Medianwerte 26

Abbildung 10: Verlauf der AP-Medianwerte 27

Abbildung 11: Verlauf der Gesamtbilirubin-Medianwerte 28

Abbildung 12: Verlauf der Wochenmediane der Transaminasen und Cholestasewerte 29

Abbildung 13: Verlauf der Quick-Wert-Medianwerte 30

Abbildung 14: Verlauf der Kreatinin-Medianwerte 31

Abbildung 15: Verlauf der Hb-Medianwerte 32

Abbildung 16: Verlauf der Leukozyten-Medianwerte 33

Abbildung 17: Verlauf der pH-Medianwerte 34

Abbildung 18: Verlauf der $\mathrm{pO}_{2}$-Medianwerte 35

Abbildung 19: Verlauf der $\mathrm{pCO}_{2}$-Medianwerte 36

Abbildung 20: Verlauf der Laktat-Medianwerte 37

Abbildung 21: Prozentuale Verteilung der diagnostischen Bildgebung 38

Abbildung 22: Verteilung der pathologischen Befunde der Abdomensonographie $\quad 40$

Abbildung 23: Verteilung der pathologischen Befunde der Computertomographie 41

Abbildung 24: Histopathologische Befunde (Leberbiopsie, Casts) 43

Abbildung 25: ERC-Befunde bei Patienten mit ischämischer Cholangiopathie $\quad 50$

Abbildung 26: Anzahl der ERC-Untersuchungen bis Diagnose 51 


\section{Tabellenverzeichnis}

Tabelle 1: Vormedikation bei Patienten mit ischämischer Cholangiopathie 16

Tabelle 2: Verlauf der Wochenmediane der Transaminasen und Cholestasewerte 29

Tabelle 3: Erregerspektrum der nachgewiesenen Keime 47

Tabelle 4: Erregerspektrum der Keime im Gallesekret $\quad 48$ 


\section{$1 \quad$ Einleitung}

\subsection{Ischämische Cholangiopathie}

Biliäre Komplikationen sind ein häufiges Problem bei Patienten nach Lebertransplantation und werden mit einer Inzidenz von über $30 \%$ angegeben. Dabei wurden als prädisponierende Faktoren u.a. Thrombosen oder Stenosen der Arteria hepatica oder chirurgische Rekonstruktionstechniken identifiziert. Diese Faktoren können $\mathrm{zu}$ ischämischen Komplikationen der Gallenwege führen (Mourad et al. 2014).

Ein ungewöhnlicher Typ der diffusen Gallenwegsschädigung, der mit multiplen intrahepatischen Strikturen, Erweiterungen der Gallenwege, Flüssigkeitsansammlungen und intrahepatischen Abszessen einhergeht, wird bei $2 \%$ der Patienten innerhalb von fünf Jahren nach Lebertransplantation beschrieben. Dieser Typ trat bei Patienten auf, die eine intakte arterielle Gefäßperfusion aufwiesen und initial keine Hinweise auf Gallengangsschädigung gezeigt hatten (Li et al. 1992; Sanchez-Urdazpal et al. 1993). Solche trotz durchgängiger A. hepatica auftretende Gallenwegsveränderungen werden als ischämische biliäre Läsionen oder ischämische Cholangiopathie bezeichnet (Chan et al. 2008). Gelegentlich wird die ischämische Cholangiopathie auch als ischämische Cholangitis bezeichnet, obwohl die Entzündung nicht die primäre Ursache zu sein scheint (Deltenre und Valla 2006).

In den letzten Jahren wurde, besonders in Fallberichten (Engler et al. 2003; Benninger et al. 2005; Gelbmann et al. 2007), ein ähnliches Krankheitsbild bei nicht lebertransplantierten, jedoch langzeitig intensivmedizinisch behandelten Patienten beschrieben. Dieses Krankheitsbild ähnelt der ischämischen Cholangiopathie und ist häufig mit einer sekundär sklerosierenden Cholangitis assoziiert.

Die sekundär sklerosierende Cholangitis ist eine chronische cholestatische Erkrankung, die mit progredienter hepatischer Fibrose, multiplen Gallenwegsstrikturen und Erweiterungen der Gallenwege einhergeht (Ruemmele et al. 2009).

Es wurden mehrere Fälle beschrieben, bei denen Patienten ohne zuvor bekannte hepatobiliäre Erkrankungen nach schwerem Trauma, Verbrennungen, größeren operativen Eingriffen und daraus resultierenden Intensivaufenthalten eine ischämische Cholangiopathie mit einer sekundär sklerosierenden Cholangitis entwickelten. In der endoskopischen retrograden Cholangiographie zeigten sich bei diesen Patienten Veränderungen der Gallenwege mit multifokalen Strikturen, Dilatationen der intrahepatischen Gallenwege oder die Präsenz von 
nekrotischem Gallenwegsmaterial (Benninger et al. 2005; Jaeger et al. 2006; Lin et al. 2014; Ben-Ari et al. 2015).

In Anbetracht der Vorgeschichte der Patienten mit einem langdauernden und komplikationsbehaftetem Intensivaufenthalt wurde hierfür auch der Begriff der „secondary sclerosing cholangitis in critically ill patients“ (SC-CIP) geprägt (Gelbmann et al. 2007; Voigtländer et al. 2012; Kirchner und Rümmele 2015; Ben-Ari et al. 2015).

\section{2 Ätiologie}

Die Ätiologie der ischämischen Cholangiopathie mit sekundär skerosierender Cholangiopathie bei Patienten mit Intensivaufenthalt (ggf. langdauernd und komplikationsbehaftet) ist unklar (Kirchner und Rümmele 2015). Möglicherweise spielt die Schädigung der Cholangiozyten, verursacht durch hypoxämische Vorgänge, eine wichtige Rolle (Gelbmann et al. 2007; Lin et al. 2014; Thomas et al. 2014).

Während das Leberparenchym eine duale Blutversorgung mit Blut aus der Arteria hepatica propria und Vena portae aufweist, erfolgt die Versorgung der Gallenwege vorwiegend über die Äste der Leberarterien, bekannt als peribiliärer Plexus. Daher reagiert das Gallenwegsepithel besonders empfindlich auf Beieinträchtigungen des arteriellen Blutflusses (Batts 1998; Ruemmele et al. 2009)

Bei Patienten mit Intensivaufenthalt und ischämischer Cholangiopathie wurde vor allem ein prolongiert erniedrigter mittlerer arterieller Druck (MAD) beobachtet. Eine daraus möglicherweise resultierende Minderperfusion der Gallenwege wird als wichtigster Faktor in der Krankheitsentstehung vermutet (Leonhardt et al. 2015). Möglicherweise spielen erhöhte Beatmungsdrücke (Gelbmann et al. 2007) oder Adipositas und längerdauernde Bauchlagerung während des Intensivaufenthaltes ebenfalls eine Rolle (Weig et al. 2012).

In manchen Fällen ist die Ätiologie der sekundär sklerosierenden Cholangitis jedoch bekannt. Sie wird u.a. durch chronische Gallenwegsobstruktion, z.B. durch Steine, durch parasitäre Erkrankungen bei immunkompromittierten Patienten (AIDS) oder immunologisch wie bei der $\mathrm{IgG}_{4}$-Cholangiopathie vermittelt (Ruemmele et al. 2009).

\subsection{Epidemiologie}

Größere Studien zur Epidemiologie der ischämischen Cholangiopathie/sekundär sklerosierenden Cholangiopathie sind derzeit nicht vorhanden. In der Mayo Clinic konnten retrospektive Auswertungen innerhalb eines Zeitraumes von 10 Jahren (1992-2002) nur 31 Patienten mit sekundär skerosierender Cholangiopathie identifizieren. Dabei zeigten diese 
Patienten eine schlechtere Prognose sowie kürzere Lebensdauer im Vergleich zu Patienten mit primär sklerosierender Cholangitis (PSC) (Gossard et al. 2005). Lin et al. (2014) beschrieben in ihrer Recherche eine Zusammenfassung von 11 Autoren aus den Jahren 20012012. 88 Patienten (davon 64 männlich) mit ischämischer Cholangiopathie und sekundär sklerosierender Cholangitis nach Intensivaufenthalt wurden zusammenfassend analysiert. In einem Follow up von ca. 18 Monaten starben 34 Patienten. 53 weitere Patienten überlebten, hiervon hatten 31 Patienten eine Leberzirrhose und waren in stabilem Zustand, während 20 Patienten für eine Lebertransplantation in Frage kamen.

\subsection{Diagnostik}

\subsubsection{Laborparameter}

In den Anfangsstadien der ischämischen Cholangipathie, ggf. kombiniert mit einer sekundär skerosierenden Cholangitis, sind klinische Symptome selten und nicht pathognomonisch. Daher sind erhöhte Laborwerte, insbesondere Cholestasewerte, häufig der einzige Hinweis für das Vorliegen dieses Krankheitsbildes (Thomas et al. 2014). Das Krankheitsbild kann sich neben den erhöhten Cholestasewerten auch mit Ikterus und/oder Cholangitis manifestieren (Kulaksiz et al. 2008; Voigtländer et al. 2012; Benninger et al. 2005). Hierbei sollten jedoch differentialdiagnostisch auch andere Ursachen wie z.B. Medikamente, die zu einem Anstieg der Cholestasewerte führen können, berücksichtigt werden.

Laborchemisch zeigt sich bei Patienten mit ischämischer Cholangiopathie und sekundär skerosierender Cholangitis besonders ein Anstieg der y-GT und AP. Zusätzlich kann es zum Ansteigen des Bilirubins kommen (Engler et al. 2003; Ruemmele et al. 2009), sodass das klinische Bild eines Ikterus entsteht. Benninger et al. (2005) beschrieben eine Erhöhung der y-GT-Werte mit einer Verdopplung in den ersten vier bis elf Tagen nach Traumaereignis mit nachfolgendem Intensivaufenthalt als erstes laborchemisches Zeichen für eine sekundär skerosierende Cholangitis bei Patienten mit ischämischer Cholangiopathie. Diese Patienten zeigten dort auch eine Erhöhung der AP und des Bilirubins in den ersten zwei Wochen, während die Transaminasen (AST, ALT) immer erst im Verlauf nach Anstieg der Cholestaseparameter stiegen.

Lin et al. (2014) beschrieben einen Anstieg der y-GT, AP und des Gesamtbilirubins um mehr als das 10-Fache der Normalwerte. Bezüglich der AST und ALT konnte nur ein leichter Anstieg auf ca. das 3-Fache beobachtet werden. 


\subsubsection{Bildgebende Verfahren}

Die transabdominale Sonographie ist häufig die erste Untersuchungsmethode, die zur Beurteilung der intra- und extrahepatischen Gallenwegserweiterung durchgeführt wird. Sie besitzt eine hohe Spezifizität zur Identifizierung von Gallenwegsobstruktionen. Die Spezifität bei Choledocholithiasis beträgt zwischen 95\%-100\% (Siddique und Kowdley 2012).

Bei Patienten mit ischämischer Cholangiopathie und sekundär sklerosierender Cholangitis nach Intensivaufenthalt ergab die transabdominale Sonographie in Abhängigkeit vom Zeitpunkt der Untersuchung unterschiedliche Befunde. In den ersten 1-2 Monaten nach Traumaereignis waren die sonographischen Befunde der Leber und Gallenwege unauffällig. Im weiteren Verlauf, nach 12-24 Monaten, kam es zum Umbau des Leberparenchyms und bei einigen Patienten auch zu zunehmenden Unregelmäßigkeiten der Gallenwege (Benninger et al. 2005).

An der A. hepatica konnten bei schwerkranken Intensivpatienten mit sekundär sklerosierender Cholangitis weder mit transabdominalem Ultraschall des Abdomens, Duplexsonographie noch Computertomographie des Abdomens Veränderungen nachgewiesen werden (Gelbmann et al. 2007).

Die Magnetresonanz-Cholangiopankreatikographie (MRCP) ist eine gängige Untersuchungsmethode bei Patienten mit Gallenwegsveränderungen im Rahmen einer primär sklerosierenden Cholangitis (PSC) (Chapman et al. 2009). Bei diesem Krankheitsbild hat die MRCP vergleichbare diagnostische Sicherheit wie die endoskopische retrograde Cholangiopankreatikographie (ERCP).

In der MRCP-Untersuchung können jedoch geringfügige Gallenwegsveränderungen in den früheren Krankheitsstadien der PSC leicht übersehen werden (Chapman et al. 2009). Die MRCP-Befunde bei der sekundär sklerosierenden Cholangitis können eine primär sklerosierende Cholangitis nachahmen. Hier können sich ebenfalls Strikturen der Gallenwege und Gallenwegsdilatationen zeigen (Vitellas et al. 2000). Ben Ari et al. (2015) beschrieben vier konsekutive Patienten mit sekundär sklerosierender Cholangitis nach langdauernder Intensivbehandlung (129 \pm 53 Tage) infolge von Verbrennungen. Bei drei dieser Patienten wurde die Diagnose mittels MRCP und Leberbiopsie gestellt. Es zeigten sich, ähnlich wie bei der primär sklerosierenden Cholangitis, Unregelmäßigkeiten der Gallenwege mit Strikturen und Dilatationen.

Benninger et al. (2005) beschrieben drei Patienten (3 von insgesamt 5) mit sekundär sklerosierender Cholangitis nach Intensivaufenthalt. Einer der Patienten hatte in einer früh durchgeführten MRT/MRCP einen unauffälligen Befund. Die MRCP bei zwei anderen 
Patienten (durchgeführt 2 Jahre nach Ereignis) zeigte bei einem der Patienten Dilatationen der intrahepatischen Gallenwege sowie Atrophie des linken Leberlappens. Bei dem anderen Patienten wurde ein zirrhotischer Umbau in der MRCP sichtbar.

\subsubsection{Leberbiopsie}

Die überwiegende Anzahl der Patienten mit sekundär sklerosierender Cholangitis nach Intensivaufenthalt wies histopathologische Auffälligkeiten in der Leberbiopsie auf (Ben-Ari et al. 2015; Esposito et al. 2008; Benninger et al. 2005).

In den frühen Phasen, nämlich in den ersten Wochen und Monaten des Krankheitsverlaufes, zeigte sich eine leichte portale Entzündung mit Lymphozyten und neutrophilen Granulozyten. Diese Entzündungsreaktion ging auch mit portalem Ödem einher. Des Weiteren zeigten sich auch Gallenwegsalterationen mit Kernhyperchromasie. Leberbiopsien in späteren Erkrankungsphasen (nach über drei Monaten) zeigten Gallengangsproliferationen, portales Ödem, portale und periduktale Fibrose sowie kanalikuläre Cholestase (Gelbmann et al. 2007; Esposito et al. 2008; Ben-Ari et al. 2015).

Biopsien nach 12 bis 14 Monaten zeigten in einigen Fällen einen kompletten zirrhotischen Umbau, in anderen Fällen wiederum nur Zeichen einer leichten portalen und duktalen Fibrose (Benninger et al. 2005; Gelbmann et al. 2007; Ben-Ari et. al 2015).

Esposito et al. (2008) konnten keine Korrelation zwischen dem Anstieg der Cholestase- und Transaminasewerten im Plasma und dem histopathologischen Befund der Leberbiopsie feststellen. So wiesen einige der Patienten mit erhöhten Transaminase- und Cholestasewerten eine geringere morphologische Schädigung der Hepatozyten sowie geringere biliäre Cholestase auf und vice versa.

\subsubsection{Endoskopische retrograde Cholangiographie (ERC)}

Morphologische Kriterien für die Diagnose einer sekundär sklerosierenden Cholangitis, die sowohl für die ERC als auch für die MRC-Bildgebung gelten, sind u.a. irreguläres Gallenwegssystem mit typischerweise unregelmäßigen intrahepatischen Gallenwegen. Es treten multiple Stenosen und Dilatationen der intrahepatischen Gallenwege auf. Weiterhin findet sich der Nachweis von „Cast“-Formationen (Gelbmann et al. 2007; Keymling et al. 2013; Albert et al. 2015; Ben-Ari et al. 2015) Mit dem Begriff „Casts“ wurden ursprünglich bei Patienten nach Lebertransplantation „Ausgüsse“ der Gallenwege mit teils kalkhaltigem Material beschrieben (Martineau et al. 1972; Waldram et al. 1975). Die Pathogenese der biliären Casts bleibt letztendlich unklar (Parry und Muiesan 2003). 
Diese Ablagerungen (Casts) in dem intrahepatischen Gallenwegssystem bestehen aus Bilirubin, Kollagen, Gallensäure, Cholesterin, verdickter Galle sowie beschädigtem Gewebe der Gallenwege (Barton et al. 1995; Gor et al. 2008).

Die ERC dient sowohl bei Patienten mit bilary cast syndrome nach Lebertransplantation als auch bei Patienten mit ischämischer Cholangiopathie und ggf. sekundär sklerosierender Cholangitis nach Intensivaufenthalt als therapeutische Maßnahme zur Entfernung der Casts aus den Gallenwegen, um den Galleabfluss zu gewährleisten (Engler et al. 2003; Gelbmann et al. 2007; Kulaksiz et al. 2008; Voigtländer et al. 2013; Mourad et al. 2014).

\subsubsection{Mikrobiologische Befunde}

Angaben zu bakteriologischen Befunden in der Blutkultur bei Erkrankten mit sekundär sklerosierender Cholangitis unter intensivmedizischer Behandlung (SC-CIP) sind kaum vorhanden. Voigtländer et al. (2015) beschrieben, dass nur 8/20 Patienten einen bakteriellen Keimnachweis in der Blutkultur hatten, während in der biliären Kultur alle diese Patienten einen positiven Keimnachweis zeigten. Ben-Ari et al. (2015) berichteten über den Nachweis von teils multiresistenten Keimen (Pseudomonas aeruginosa, Enterococcus faecalis, MRSA, Acinetobacter) bei Patienten mit sekundär sklerosierender Cholangitis und Intensivaufenthalt. Scheppach et al. (2001) beschrieben drei Patienten mit systemischen Infektionen, Intensivaufenthalt und Nachweis von Streptococcus pyogenes, Streptococcus aureus und Streptococcus pneumonie (in Mediastinalgewebe, kutanem Abszess bzw. Bronchiallavage), bei denen die Entwicklung zu einer sklerosierender Cholangitis möglicherweise im Zusammenhang mit der systemischen Infektion steht.

Mikrobiologische Analysen der Galle, gewonnen im Rahmen von ERC-Untersuchungen, bei Patienten mit biliary cast syndrome nach Lebertransplantation oder bei Intensivpatienten mit sekundär sklerosierender Cholangitis zeigten häufig Nachweise von Candida spp., Enterococcus spp. und Pseudomonas aeruginosa. Immer wieder erwiesen sich die Erreger als multiresistent (Gelbmann et al. 2007; Voigtländer et al. 2013; Voigtländer et al. 2015).

\subsection{Therapie}

Die gängigste diagnostische wie auch therapeutische Option bei Patienten mit ischämischer Cholangiopathie, ggf. in Kombination mit sklerosierender Cholangitis, stellt die ERC dar. Im Rahmen der ERC wird meist eine endoskopische Papillotomie (EPT) mit Entfernung von Cast-Material durchgeführt (Gelbmann et al. 2007; Kulaksiz et al. 2008; Voigtländer et al. 2012; Voigtländer et al. 2013). In einigen Fällen wird auch die Anlage einer nasobiliären Drainage mit Spülung über mehrere Tage als therapeutische Maßnahme beschrieben 
(Gelbmann et al. 2007). Viele der Patienten benötigen mehrfache ERC-Interventionen (Jaeger et al. 2006). Die endoskopische Therapie mittels ERC führt zur Verbesserung der klinischen Parameter und laborchemisch zu rückläufigen Cholestasewerten (Ruemmele et al. 2009).

Im späteren Verlauf des Krankheitsbildes mit zirrhotischem Umbau des Leber- und Gallengangsgewebes kann eine endoskopische Behandlung meist nur kurzfristig die Leberfunktion verbessern. Dabei kann ein Fortschreiten der Erkrankung jedoch nicht verhindert werden (Engler et al. 2003; Kulaksiz et al. 2008).

Die Gewinnung von Galleflüssigkeit im Rahmen der ERC zur mikrobiologischen Analyse wird aufgrund von häufig auftretenden multiresistenten Keimen zur gezielten antimikrobiellen Behandlung empfohlen (Voigtländer et al. 2015).

Eine Kombinationstherapie aus Ursodesoxycholsäure (UDC) und endoskopischen Interventionen mittels ERC wird von mehreren Autoren beschrieben (Benninger et al. 2005; Gelbmann et al. 2007; Kulaksiz et al. 2008; Ben-Ari et al. 2015). Diese Behandlung scheint bei den meisten Patienten kurzfristig zu einer Verbesserung der Cholestase zu führen (Engler et al. 2003; Lin et al. 2014). Kulaksiz et al. (2008) konnten die Effizienz der UDC-Therapie nicht bestätigen.

Aufgrund der Progredienz der Erkrankung und der zirrhotischen Umbauprozesse der Leber muss die Lebertransplantation ggf. als kurative Option bei Patienten in Betracht gezogen werden (Voigtländer et al. 2012; Lin et al. 2014; Ben-Ari et al. 2015; Kirchner und Rümmele 2015).

\subsection{Prognose}

Aufgrund des Fortschreitens der Erkrankung der ischämischen Cholangiopathie mit Entwicklung einer sekundär biliären Zirrhose ist die Prognose der Krankheit schlecht.

Engler et al. (2003) berichteten, dass von insgesamt neun Patienten fünf starben (Follow up 35 Monate im Median). Vier der neun Patienten entwickelten eine Leberzirrhose. Gelbmann et al. (2007) beschrieben 26 Patienten mit einem Follow up von 4-233 Tagen. Hiervon benötigten vier der Patienten eine Lebertransplantation. Sechs Patienten verstarben an Multiorganversagen. 10 der Patienten hatten eine sklerosierende Cholangitis und moderate Cholestasewerte. Bei Kulaksiz et al. (2008) (28 Patienten) betrug der Follow up 13 Jahre und das Überleben ohne Transplantation lag bei 13 Monaten im Median. 
Voigtländer et al. (2012) berichteten über 54 Patienten (Follow up 165 Tage nach ERCDiagnose). Während des Follow up starb die Hälfte der Patienten (29 der 54 Patienten) und sechs Patienten wurden lebertransplantiert.

Dabei scheinen Leberversagen sowie Sepsis mit Multiorganversagen die häufigsten akuten Todesursachen zu sein (Engler et al. 2003; Gelbmann et al. 2007; Kulaksiz et al. 2008; BenAri et. al 2015). 


\section{$2 \quad$ Fragestellung}

Im Rahmen dieser Arbeit erfolgte eine retrospektive Analyse eines Patientenkollektivs der Universitätsmedizin Göttingen (UMG), bei der Patienten im Zuge der Abklärung einer unklaren Hepatopathie ggf. mit Cholestase und Ikterus mittels ERC die Diagnose der ischämischen Cholangiopathie bzw. der daraus resultierenden sekundär sklerosierenden Cholangitis (SSC) gestellt worden war. Das Patientenkollektiv bestand aus Intensivpatienten mit vorwiegend schwerem Krankheitsverlauf. Patienten mit Z.n. Lebertransplantation waren nicht eingeschlossen.

Das Hauptziel der vorliegenden retrospektiven Untersuchung bestand darin, folgende Parameter zu charakterisieren und zu analysieren:

- Vorerkrankungen

- Risikofaktoren des Kollektivs

- Analyse des ,initialen Ereignisses“, das letztlich zu einer intensivmedizinischen Behandlung führte

- Analyse des Intensivaufenthaltes hinsichtlich verschiedener Parameter (u. a. Pharmokatherapie, Beatmung, laborchemische und mikrobiologische Diagnostik, Bildgebung)

- Zeitpunkt der Diagnosesicherung einer ischämischen Cholangiopathie

Die Fragestellung beinhaltet nicht die Analyse des Verlaufs bzw. des Outcomes sowie die Pharmokotherapie bei dieser Patientengruppe nach erfolgter Diagnosestellung. 


\section{Material und Methoden}

\subsection{Patientenkollektiv}

Bei vorliegender Untersuchung handelt es sich um eine rein deskriptive retrospektive Analyse. Das Kollektiv der Patienten setzt sich aus 51 Patienten der Universitätsmedizin der Georg-August-Universität Göttingen zusammen. Es handelt sich dabei entweder um Erkrankte, die in den Jahren 04/2007 bis 05/2015 in der UMG primär diagnostiziert wurden oder Patienten, bei denen nach primärer Diagnosestellung in externen Krankenhäusern die Diagnosebestätigung inclusiver Weiterbetreuung in der UMG erfolgte.

\subsection{Datenerhebung}

Die initiale Identifizierung der an dem Krankheitsbild „ischämische Cholangiopathie“ erkrankten Patienten erfolgte mittels Recherche der endoskopischen Befunddatenbank der Klinik für Gastroenterologie und Gastrointestinale Onkologie. Anschließend erfolgte eine Recherche anhand der UMG-Akten der zuvor identifizierten Patienten. Zu diesem Zweck wurden die Akten aus dem Patientenarchiv der UMG angefordert. Dabei wurden Unterlagen der Kliniken Innere Medizin, Anästhesiologie, Neurologie und Neurochirurgie ausgewertet. Fehlende Informationen zu Patienten wurden den vorliegenden Arztbriefen entnommen. Ferner wurden in bestimmten Fällen zwecks Ergänzung der Daten die Hausärzte der Kranken sowie die mitbehandelnden auswärtigen Krankenhäuser hinzugezogen.

Wenn möglich, wurde auch vorzugsweise eine Eigenanamnese der Patienten vorgenommen. In den Fällen, in denen dies nicht suffizient möglich war, erfolgte eine Fremdanamnese mit Hilfe Angehöriger oder Betreuer.

Über die endoskopischen Befunde hinaus wurden unter Zuhilfenahme der hausinternen Datenbanken der UMG sämtliche mikrobiologischen, histopathologischen und bildgebenden Befunde sowie Laborwerte eruiert. Bei auswärtigen Patienten erfolgte die Erhebung dieser Daten ebenfalls wie oben beschrieben.

Die Dokumentation aller Patientenangaben und -daten erfolgte in anonymisierter tabellarischer Form mittels Microsoft Excel 2013.

Im Rahmen der vorliegenden Arbeit wurde auf eine statistische Auswertung verzichtet. Es erfolgte eine rein deskriptive Analyse der Daten unter Angabe der Medianwerte oder Mittelwerte und Standardabweichung. Diese wurden mittels Windows Excel und Statistikprogramm IBM SPSS Statistics 20 berechnet. 


\subsection{Laborparameter}

Folgende Laborparameter wurden berücksichtigt:

- Transaminasenwerte: AST, ALT

- Cholestasewerte: AP, y-GT und Gesamtbilirubin.

- Gerinnung: Quick-Wert

- Kreatinin

- Hämoglobin und Leukozyten im kleinen Blutbild

Dabei wurden die oben genannten Parameter, wenn möglich, vom ersten Tag des initialen Krankheitsereignisses (im Folgenden als Tag 1 bezeichnet) an bis zum Zeitpunkt der Diagnosesicherung, maximal aber bis zum Tag 42 nach dem Initialereignis, ausgewertet.

Weiterhin wurden Parameter der arteriellen Blugasanalysen berücksichtigt:

- $\mathrm{pH}, \mathrm{pO}_{2}, \mathrm{pCO}_{2}$ und Laktat

Diese wurden im Rahmen einer arteriellen Blutgasanalyse-Bestimmung im Zeitraum vom ersten Tag des Initialereignisses bis maximal zum 30. Tag erhoben. Aufgrund von wiederholt durchgeführten BGAs im Intensivalltag wurde zur Auswertung der erste bestimmte Wert am ersten Tag des Intensivaufenthaltes verwendet. Im weiteren Verlauf wurden die Werte der arteriellen BGA verwendet, die auf ICU-Stationen routinemäßig jeden Morgen um 4.00 Uhr als erster Wert des Tages bestimmt wurden.

Auch die Dokumentation Laborparameter erfolgte in anonymisierter Form in Microsoft Excel Tabellen.

Nachfolgend wurden für jeden einzelnen Aufenthaltstag Medianwerte des Patientenkollektivs mit Hilfe von Windows Excel 2013 berechnet und tabellarisch und graphisch dargestellt.

\subsection{Bildgebende Verfahren}

Die Befunde folgender bildgebender Verfahren wurden im Rahmen dieser Arbeit vor Diagnosestellung analysiert:

- Abdominelle Sonographie, ggf. mit Dopplersonographie

- Endosonographie des oberen Gastrointestinaltraktes (EUS)

- Computertomographie (CT)

- Positronen-Emissions-Tomographie (PET-CT)

- Magnetresonanz-Tomographie (MRT) des Abdomens

- Magnetresonanz- Cholangiopankreatikographie (MRCP) 
Dabei wurde zunächst die Anzahl der jeweiligen Untersuchungen bestimmt. Zur Auswertung wurde die zeitlich letzte Untersuchung vor Diagnosestellung des Krankheitsbildes „Ischämische Cholangiopathie“ herangezogen.

Die Auswertung beinhaltete, soweit vorhanden, Befunde der Leber wie Beschaffenheit, Kontur, Raumforderungen, eventueller Gewebsumbau sowie Angaben zu den Lebergefäßen wie Pfortader, Lebervenen und Leberarterien. Dabei wurden auch dopplersonographische Befunde der Lebergefäße berücksichtigt. Darüber hinaus wurden Informationen über Anatomie, mögliche Veränderungen und pathologische Befunde der intra- und extrahepatischen Gallenwege sowie der Gallenblase gewonnen. Auch diese Angaben wurden anonymisiert tabellarisch in das Microsoft Excel Programm eingegeben. Dabei wurde für jede einzelne Untersuchungsmethode eine eigene Tabelle angelegt.

\subsection{Mikrobiologische Befunde}

Ausgewertet wurden die mikrobiologischen Befunde vom Zeitpunkt des initialen Krankheitsereignisses bis zum Zeitpunkt der Diagnosesicherung. Dabei wurden bakteriologische Befunde der Blutkulturen, Urinkulturen, Stuhlkulturen und Liquorkulturen analysiert. Weiterhin wurden, falls vorhanden, serologische Befunde in Blut und Liquor berücksichtigt. Des Weiteren erfolgte auch eine Beurteilung der bakteriologischen und mykologischen Befunde in der Galleflüssigkeit, die mittels ERC durch Aspiration aus den Gallenwegen gewonnen wurde. Die Dateneingabe erfolgte in Microsoft Excel tabellarisch anonymisiert und nach vorhandenem Keim- und Erregerspektrum untergliedert.

\subsection{Histopathologische und zytopathologische Befunde}

Es erfolgte eine Auswertung der histopathologischen Befunde der Leberbiopsie. Weiterhin erfolgte die Auswertung der zytopathologischen Befunde von Material, das während der ERC gewonnenen wurde. Dabei wurden alle Befunde berücksichtigt, die bis zum Zeitpunkt der Diagnosesicherung oder im Rahmen der Abklärung des Krankheitsbildes durchgeführt worden waren und dokumentiert waren.

\subsection{ERC-Befunde}

In manchen Erkrankungsfällen waren bis zur eindeutigen Diagnosestellung „Ischämische Cholangiopathie“ mehrere ERC-Untersuchungen erforderlich. Für die Datenauswertung wurden alle ERCs berücksichtigt, die bis zum Zeitpunkt der Diagnosestellung stattgefunden hatten. Die Dokumentation erfolgte anonymisiert in Windows Excel 2013. 


\section{Ergebnisse}

\subsection{Alter und Geschlechtsverteilung}

Das Gesamtkollektiv der Patienten mit ischämischer Cholangiopathie der UMG (04/200705/2015), das retrospektiv analysiert wurde, bestand aus 51 Patienten. Von diesen 51 Erkrankten waren 31 Patienten männlich und 20 Patienten weiblich. Das mittlere Alter lag bei 59,6 \pm 14,2 Jahren. Der jüngste Patient war 19 Jahre alt, der älteste Patient 83 Jahre.

Bei den männlichen Patienten zeigte sich bei Diagnosestellung ein Altersgipfel in der Gruppe der 61- bis 70-Jährigen sowie der 71-80-Jährigen mit jeweils 10 Patienten. Bei den Frauen lag der Altersgipfel bei Diagnosestellung den bei 61-70 Jahren (Abbildung 1).

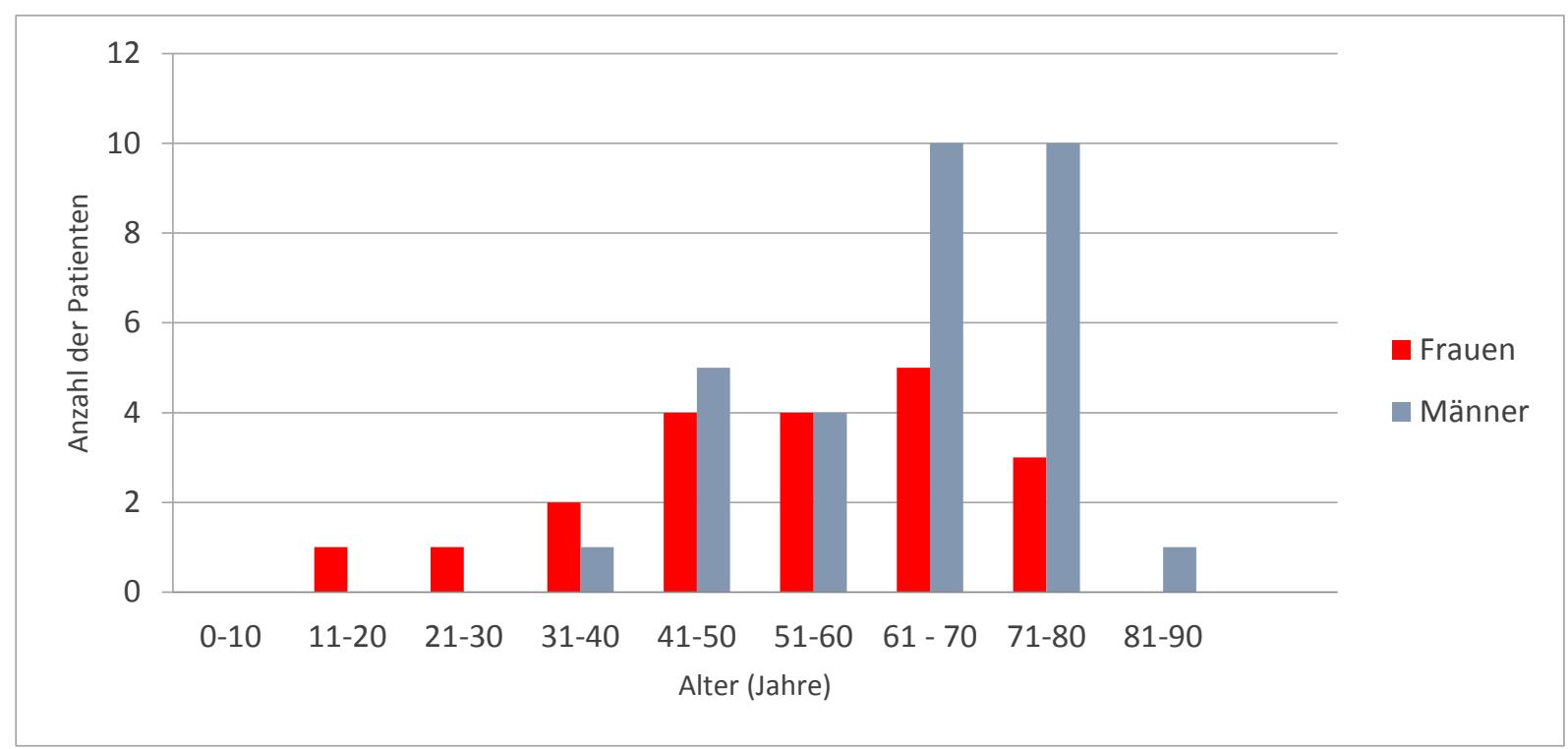

Abbildung 1: Altersverteilung der Patienten mit ischämischer Cholangiopathie der UMG (04/2007-05/2015), (Kollektiv: $n=51)$

\subsection{Vorerkrankungen}

Anhand vorliegender Patientenakten konnten unterschiedliche Vorerkrankungen bei 42 von 51 Patienten $(82,3 \%)$ ermittelt werden. Darunter waren auch Patienten, bei denen zwei oder mehr Vorerkrankungen vorlagen.

Bei den kardiovaskulären Vorerkrankungen wiesen 24 der 42 Patienten $(57,1 \%)$ einen arteriellen Hypertonus auf. Das war gleichzeitig auch die am häufigsten vorkommende Erkrankung. 9 von 42 (21,4\%) Patienten hatten eine Herzinsuffizienz unterschiedlicher Genese, 8 von 42 Patienten (19\%) hatten eine koronare Herzkrankheit. Eine pAVK oder 
Herzklappenvitien unterschiedlichen Ausprägungsgrades hatten je 5 von 42 Patienten $(11,9 \%)$. Bei 3 von 42 Patienten $(7,1 \%)$ war Vorhofflimmern vorbekannt gewesen.

Erkrankungen des endokrinen Systems und Stoffwechselkrankheiten zeigten folgende Verteilung: 14 von 42 Patienten (33,3\%) mit Hyperlipoproteinämie, 12 von 42 Patienten (28,5\%) mit Diabetes mellitus, 9 von 42 Patienten (21,4\%) mit Adipositas, 4 von 42 Patienten (9,5\%) mit Hyperurikämie, 2 von 42 Patienten (4,7\%) mit Autoimmunthyreoiditis und jeweils ein Patient mit Hyper- bzw. Hypothyreose (je 2,3\%).

Bei den neurologischen Vorerkrankungen fanden sich 2 von 42 Patienten (4,7\%) mit transitorischer ischämischer Attacke (TIA) in der Vorgeschichte, 2 von 42 Patienten (4,7\%) mit Apoplex, 1 von 42 Patienten (2,3\%) mit Myasthenia gravis, 1 Patient (2,3\%) mit Epilepsie und 1 weiterer Patient (2,3\%) mit stattgehabtem Schädel-Hirn-Trauma.

7 von 42 Patienten $(16,6 \%)$ hatten eine chronische Niereninsuffizienz in der Vorgeschichte.

Bei 5 von 42 Patienten $(11,9 \%)$ fanden sich pulmonale Vorerkrankungen. Hiervon war COPD bei 3 von 42 Patienten (7,1\%), obstruktives Schlaf-Apnoe-Syndrom (OSAS) und pulmonal arterielle Hypertonie bei 1 Patienten (je 2,3\%) nachweisbar.

Onkologische Vorerkrankungen ließen sich bei 5 der 42 Patienten (11,9\%) eruieren. (Mammakarzinom, Prostatakarzinom, Polycythämia vera, Non Hodgkin Lymphom und Bronchialkarzinom).

Psychiatrische Vorerkrankungen waren bei 3 der 42 Patienten $(7,1 \%)$ beschrieben, nämlich depressive Störungen bei 2 von 42 Patienten (4,7\%) sowie eine schizoide Störung bei 1 von 42 Patienten $(2,3 \%)$.

Bei 3 von 42 Patienten $(7,1 \%)$ waren je eine rheumatologische Erkrankung vorbekannt (chronisch entzündliche Darmerkrankungen (CED), Lupus erythematodes, rheumatoide Arthritis).

Hepatologische Vorerkrankungen fanden wir bei 1 von 42 Patienten (2,3\%) (Leberzirrhose). Bei 1 der 42 Patienten (2,3\%) war ein Marfan-Syndrom vorbeschrieben.

Die Vorerkrankungen, die nach Aktenlage ermittelt werden konnten (betrifft 42 von 51 Patienten, 82,3\%), wurden ihrer jeweiligen Krankheitsentität entsprechend zusammengefasst. Dabei zeigte sich, dass kardiovaskuläre Krankheitsbilder am häufigsten vorkamen (54 Krankheitsfälle). An zweiter Stelle standen die Erkrankungen des endokrinen Systems und Stoffwechsels mit 43 Fällen. Die weitere Verteilung stellte sich in absteigender Häufigkeit folgendermaßen dar: neurologische Vorerkrankungen mit 7 Krankheitsfällen, renale Vorerkrankungen mit ebenfalls 7 Fällen, pulmonale und neoplastische Vorerkrankungen mit 
je 5 Krankheitsfällen. Des Weiteren waren 3 Krankheitsfälle rheumatologisch und 3 weitere Fälle psychiatrisch einzuordnen. Hepatologische Vorerkankungen fanden sich selten (1 Patient). Zuletzt fand sich ein als sonstiger Fall beschriebenes Marfan-Syndrom (Abbildung 2).

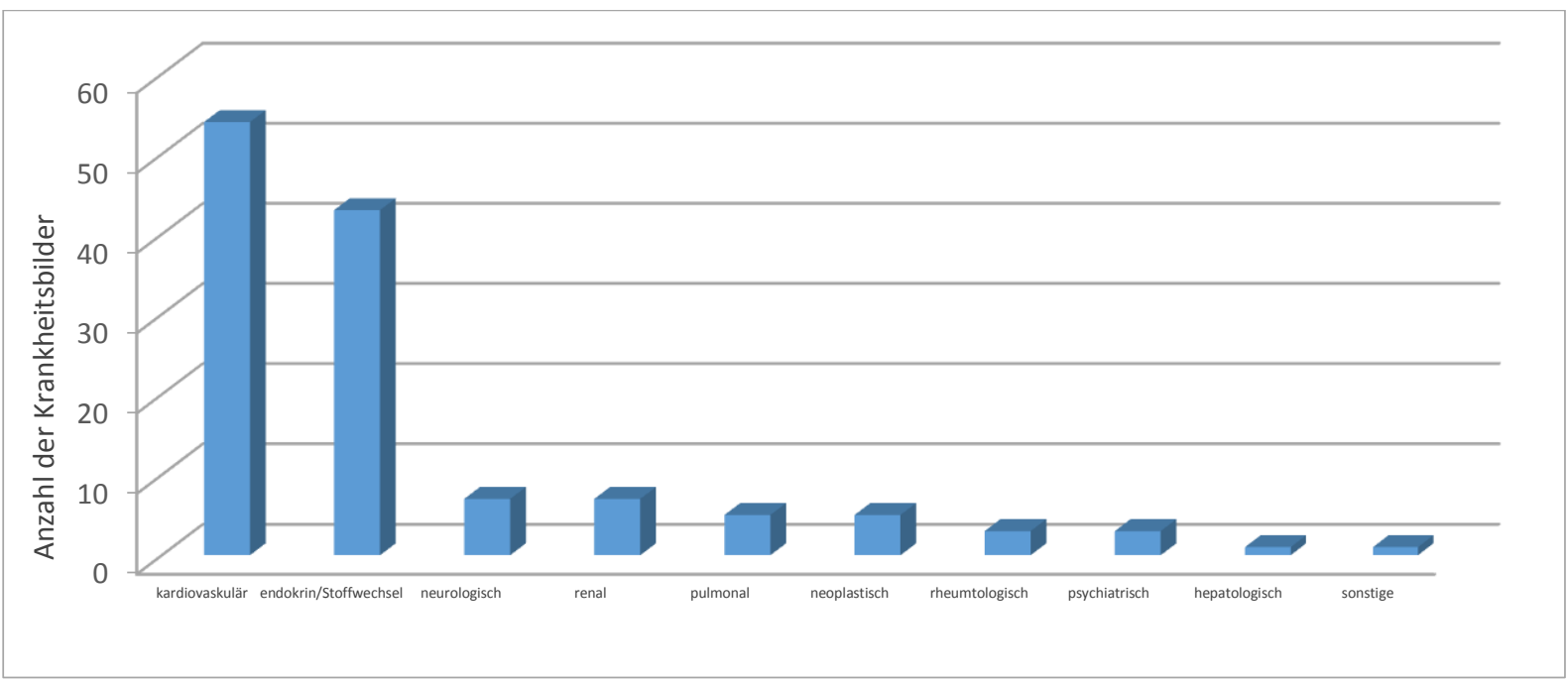

Abbildung 2: Verteilung der Vorerkrankungen bei Patienten mit ischämischer Cholangiopathie der UMG (04/2007-05/2015), (Kollektiv: $\mathrm{n}=42$ von 51 Patienten)

\subsection{Anamnestische Risikofaktoren}

Bei 42 von 51 (82,3\%) Patienten konnten aufgrund der Aktenlage neben Angaben zu den Vorerkrankungen auch anamnestische Angaben zu Risikofaktoren erhoben werden. Darunter fanden sich in den Patientenunterlagen 9 von 42 Patienten $(21,4 \%)$ mit langanhaltendem Nikotinkonsum, 7 von 42 Patienten (16,6 \%) mit chronischem Alkoholkonsum sowie 3 von 42 Patienten $(7,1 \%)$ mit Cannabiskonsum.

\subsection{Vormedikation}

Die Vormedikation konnte bei 32 von insgesamt 51 Patienten $(62,7 \%)$ ermittelt werden. Die am häufigsten eingesetzten Pharmakagruppen waren Diuretika (50\%), ACE-Hemmer/AT1Antagonisten und Thrombozytenaggregationshemmer (je 40,6\%), $\beta$-Blocker $(37,5 \%)$, Protonenpumpeninhibitoren sowie Lipidsenker (je 28,1\%) und Psychopharmaka (25\%).

Weitere Arzneigruppen, die bei Patienten in der Vormedikation vorhanden gewesen waren, sind in Tabelle 1 aufgeführt. 
Tabelle 1: Vormedikation bei Patienten mit ischämischer Cholangiopathie der UMG (04/2007-05/2015), (Kollektiv n=32 von 51 Patienten)

\begin{tabular}{|l|l|}
\hline Pharmakagruppe & $\begin{array}{l}\text { Anzahl der Patienten und } \\
\text { prozentuale Häufigkeit }\end{array}$ \\
\hline Diuretika & $16 / 32(50 \%)$ \\
\hline ACE-Hemmer/AT1-Antagonisten & $13 / 32(40,6 \%)$ \\
\hline Thrombozytenaggregationshemmer & $13 / 32(40,6 \%)$ \\
\hline B-Blocker & $12 / 32(37,5 \%)$ \\
\hline Protonenpumpeninhibitoren & $9 / 32(28,1 \%)$ \\
\hline Lipidsenker & $9 / 32(28,1 \%)$ \\
\hline Psychopharmaka & $8 / 32(25 \%)$ \\
\hline Insulin & $6 / 32(18,7 \%)$ \\
\hline Ca-Antagonisten (Nifedipin- und Verapamil-Typ) & $5 / 32(15,6 \%)$ \\
\hline Orale Antikoagulation & $3 / 32(9,3 \%)$ \\
\hline Niedermokekulare Heparine & $3 / 32(9,3 \%)$ \\
\hline Orale Antidiabetika & $3 / 32(9,3 \%)$ \\
\hline Urikostatika & $3 / 32(9,3 \%)$ \\
\hline Inhalative Bronchodilatatoren & $3 / 32(9,3 \%)$ \\
\hline Kortikosteroide & $2 / 32(6,2 \%)$ \\
\hline Nitrate und weitere Dilatatoren & $2 / 32(6,2 \%)$ \\
\hline
\end{tabular}

\subsection{Initiales Krankheitsereignis}

Als initiales Krankheitsereignis wurde der Zeitpunkt festgelegt (Tag, Monat und Jahr), an dem die Patienten einen Vorfall hatten, der zum Intensivaufenthalt und im weiteren Verlauf zum pathologischen Anstieg der Cholestasewerte und letztendlich zur Diagnosestellung führte.

Diese Ereignisse wurden anhand der Krankheitsentitäten in sieben Kategorien eingeteilt und nach der Häufigkeit des Vorkommens graphisch dargestellt (Abbildung 3). Der ersten Kategorie wurden die Patienten mit intrakraniellen Blutungen (ICB) zugewiesen. Dies betraf 24 von 51 Patienten $(47 \%)$. 
In der zweiten Kategorie befanden sich Patienten mit primär pulmonalem Ereignis und respiratorischer Dekompensation bei Pneumonie, acute respiratory distress syndrome (ARDS) oder Lungenarterienembolie. Von diesen Erkrankungen waren 9 der 51 Patienten $(17,6 \%)$ betroffen.

Die dritte Kategorie betraf 7 von 51 Patienten $(13,7 \%)$ mit zuvor statt gehabten operativen Eingriffen (Herzklappen-Operationen, aortokoronare Venenbypass-Operation (ACVB-OP), sonstige Gefäßoperationen).

Der vierten Kategorie wurden 4 von 51 Patienten $(7,8 \%)$ zugeordnet, bei denen ein kardiales Initialereignis wie akutes Koronarsyndrom (ACS) und/oder Aortendissektion ohne primär operative Versorgung statt gefunden hatte.

In der fünften Kategorie befanden sich 4 von 51 Patienten $(7,8 \%)$ mit Hirninfarkt.

Die sechste Kategorie bezog sich auf 2 von 51 Patienten (3,9\%) mit ZNS-Entzündung.

In der letzten Gruppe befand sich 1 von 51 Patienten (1,9\%) mit Volumenmangel nach Massenblutung.

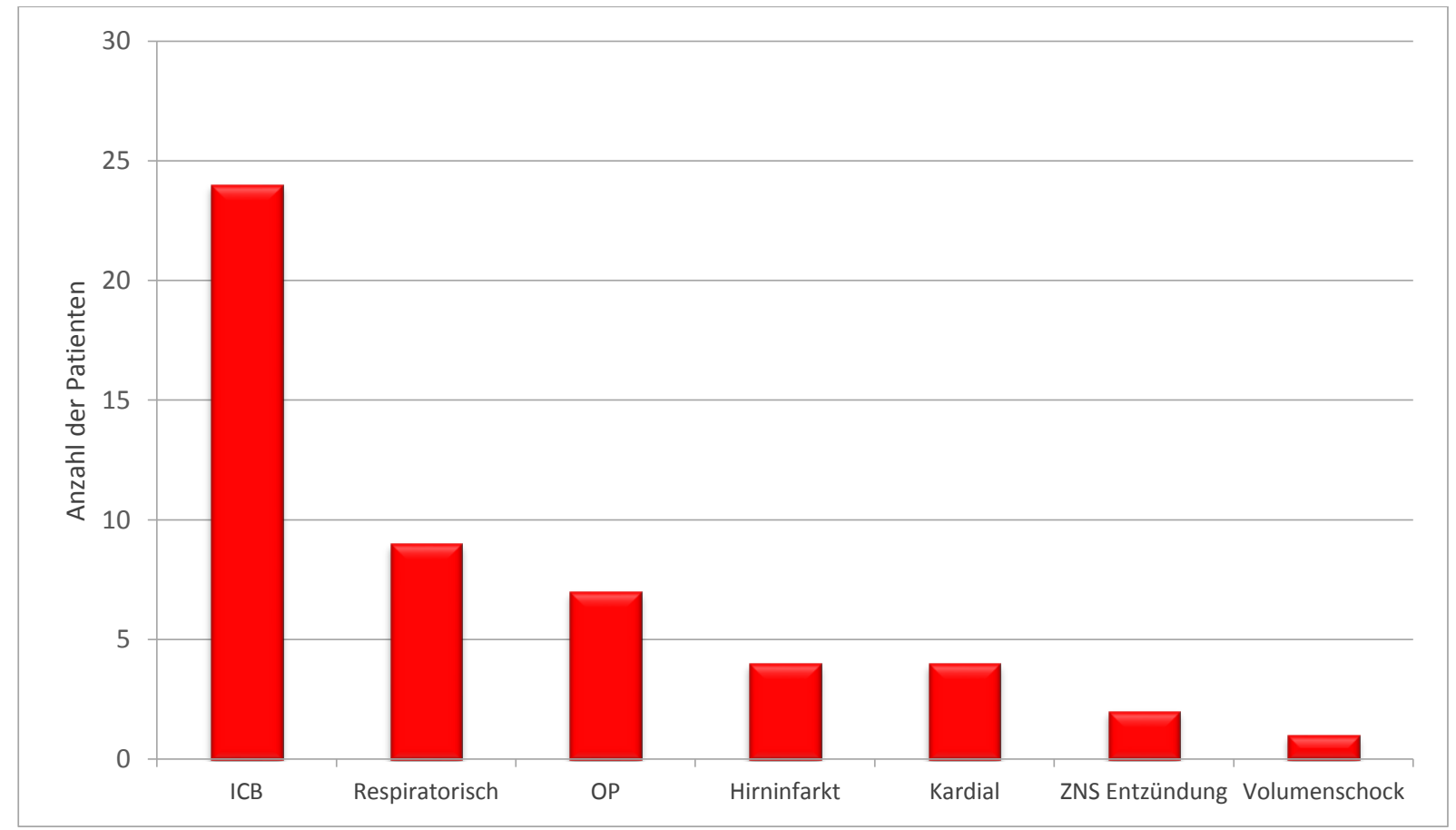

Abbildung 3: Darstellung der Ereignisverteilung bei Patienten mit ischämischer Cholangiopathie der UMG (04/2007-05/2015), (Kollektiv: $n=51)$ 


\subsection{Aufenthalt auf Intensivstation}

Der Diagnose des Krankheitsbildes „Ischämische Cholangiopathie“ war bei all unseren Patienten ein initiales Ereignis mit nachfolgendem Intensivaufenthalt voraus gegangen.

Von 51 Patienten hatten 42 Patienten (82,3\%) einen Intensivaufenthalt in der Universitätsmedizin Göttingen (UMG) und 9 von 51 Patienten (17,7\%) einen längeren Intensivaufenthalt in auswärtigen Krankenhäusern hinter sich.

Bei 29 von 42 Patienten (69\%) mit Intensivaufenthalt in der UMG handelte es sich um anästhesiologische Intensivaufenthalte. 7 von 42 Patienten (17,7\%) verbrachten einen Intensivaufenthalt auf einer neurologischen Intensivstation und 3 von 42 Patienten $(5,9 \%)$ auf einer internistischen Intensivstation. 3 der 42 Patienten $(7,1 \%)$ waren im Verlauf auf mehreren Intensivstationen der UMG betreut.

Anhand der Unterlagen konnte ermittelt werden, dass 3 von 42 Patienten $(7,1 \%)$ vor Aufenthalt in der UMG kardiopulmonal reanimiert worden waren. Im Rahmen des Intensivaufenthaltes in der UMG war nur ein Fall mit Kreislaufstillstand und anschließender kurzfristiger Reanimation dokumentiert.

30 von 42 Patienten $(71,4 \%)$ waren bei Aufnahme auf einer Intensivstation der UMG intubiert. Bei 10 von 42 Patienten $(23,8 \%)$ erfolgte die Aufnahme im wachen Zustand. Bei 2 von 42 Patienten $(4,7 \%)$ war der Zustand bei Aufnahme in den vorhandenen Unterlagen nicht dokumentiert.

Bei 20 von 51 Patienten (39,3\%) lagen Angaben zur Dauer der invasiven Beatmung vor. Darunter zeigte sich ein Medianwert von 22 Tagen mit invasiver Beatmung (minimal: 9 Tage, maximal: 72 Tage).

Bei 44 von insgesamt 51 Patienten (86,2\%) lag der Intensivaufenthalt im Median bei 32 Tagen mit einem Minimum von 13 Tagen und einem Maximum von 145 Tagen.

Der Intensivaufenthalt lag bei 4 der 51 Patienten (7,8\%) bei 1-15 Tagen, bei 13 von 51 Patienten $(25,5 \%)$ waren es $16-30$ Tage und bei 17 von 51 Patienten $(33,3 \%)$ waren es 31-45 Tage.

Bei 6 von 51 Patienten (11,7\%) lag die Dauer des intensivstationären Aufenthaltes bei 46 60 Tagen und bei 4 von 51 Patienten $(7,8 \%)$ bei mehr als 90 Tagen.

Bei 7 von insgesamt 51 Patienten (13,7\%) konnte anhand der Akten die Dauer des Intensivaufenthaltes nicht ermittelt werden. 


\subsubsection{Invasive Maßnahmen während des Intensivaufenthaltes}

Bei 47 von 51 Patienten $(92,1 \%)$ konnten Daten über die invasiven Maßnahmen während des Intensivaufenthaltes ausgewertet werden. Davon hatte der Großteil, nämlich 34 von 47 Patienten (72,3\%), eine Tracheotomie erhalten. 21 von 47 Patienten $(44,6 \%)$ waren mit intrazerebralen Drainagen und Sonden versorgt worden, bei 16 von 47 Patienten (34\%) waren neurochirurgische operative Eingriffe durchgeführt worden (Trepanationen, Hemikraniektomien). 14 von 47 Patienten $(29,7 \%)$ hatten eine Hämodialyse oder continuous veno-venous hemofiltration (CVVH) erhalten.

Bei 12 von 47 Patienten (25,5\%) waren Operationen des kardiovaskulären Systems (ACVBOP‘s, Herzklappenoperationen oder sonstige vaskuläre Eingriffe) vorgenommen worden.

Bei 9 von 47 Patienten $(19,1 \%)$ waren abdominalchirurgische Eingriffe (Laparotomie, Cholezystektomie, Blutstillung) durchgeführt worden und bei 7 von 47 Patienten $(14,8 \%)$ war die Anlage einer PEG-Sonde erfolgt.

Jeweils 4 von 47 Patienten $(8,5 \%)$ waren mit einer intraaortalen Ballonpumpe (IABP) und mit extrakorporaler Membranoxigenierung (ECMO) behandelt worden. 3 von 47 Patienten $(6,3 \%)$ hatten Herzkatheter-Untersuchungen. 2 von 47 Patienten (4,2\%) waren mittels Hochfrequenzoszillations-Beatmung (HFO) beatmet worden (Abbildung 4).

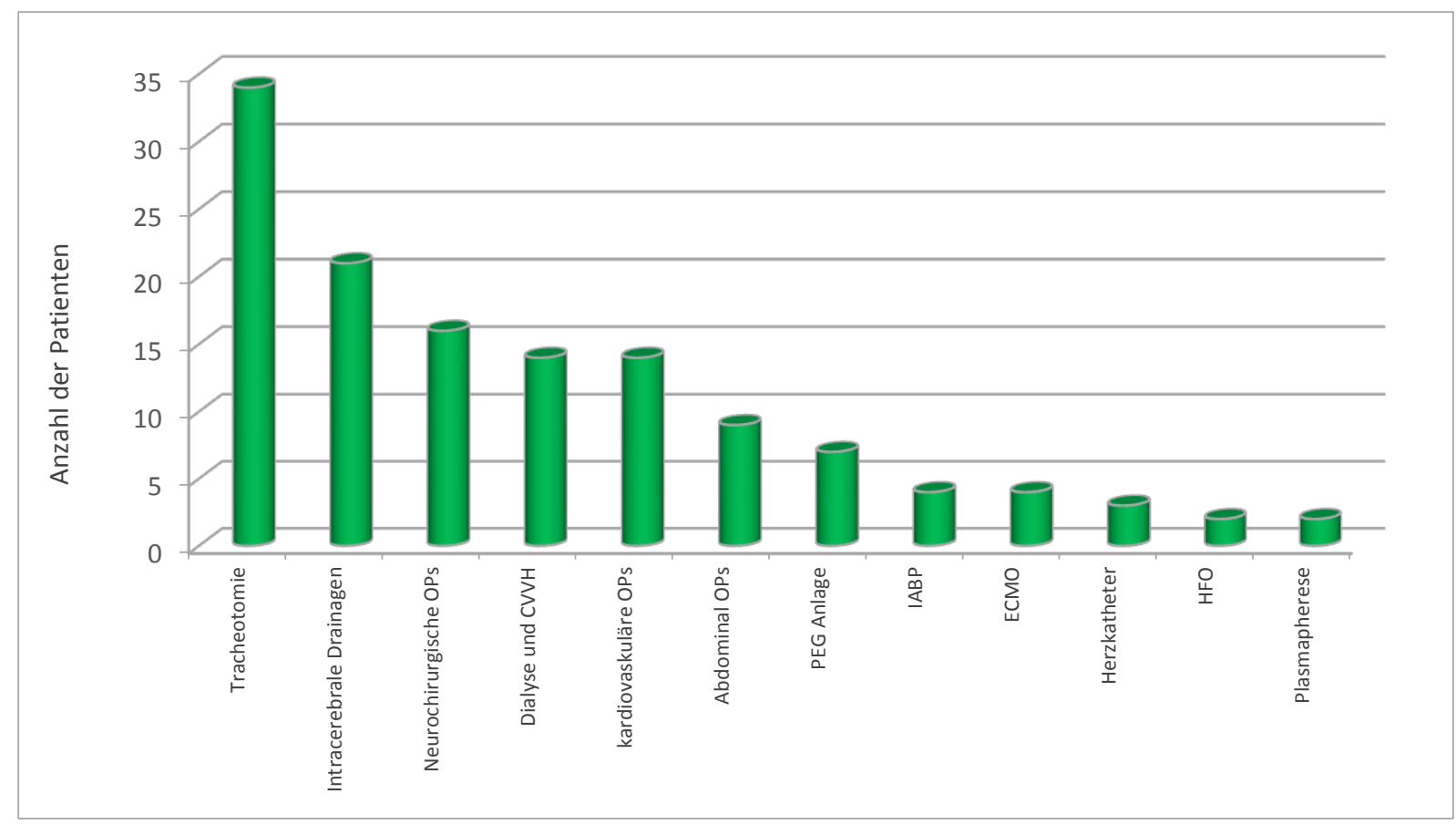

Abbildung 4: Invasive Maßnahmen während des Intensivaufenthaltes bei Patienten mit ischämischer Cholangiopathie der UMG (04/2007-05/2015), (Kollektiv: $\mathrm{n}=47$ von 51 Patienten) 


\subsubsection{Intensivmedikamentöse Therapie}

\subsubsection{Sedierung und Katecholamine während des Intensivaufenthaltes}

28 von 42 Patienten $(66,6 \%)$, die auf einer Intensivstation der UMG behandelt wurden, erhielten nach den Informationen der vorliegenden Unterlagen Ketamin-Anästhesie. Bei 26 von 42 Patienten $(61,9 \%)$ wurde eine Therapie mit Midazolam durchgeführt. 17 von 42 Patienten (40,4\%) erhielten Opioide (Sufentanil, Remifentanil, Fentanyl) (Abbildung 5). 9 von 42 Patienten $(21,4 \%)$ erhielten Propofol. Bei 5 von 42 Patienten $(11,9 \%)$ ließ sich die Sedierung/Anästhesie nach Aktenlage nicht genau eruieren 23 von 42 Patienten $(54,7 \%)$ erhielten eine Kombinationstherapie mit mindestens drei Pharmaka, meist unter zusätzlicher Hinzunahme eines Opioids. Als häufigste Dreifachkombination waren Ketamin mit Midazolam und Sufentanil vertreten (15 von 23 Patienten, 65,2\%).

Eine Kreislaufunterstützung mit Katecholaminen erhielten nach Aktenlage 23 von 42 Patienten (54,7\%). Am häufigsten wurde Noradrenalin eingesetzt (23 von 42 Patienten, 54,7\%). Bei jeweils 2 von 23 Patienten (8,6\%) wurde eine Kombinationsmedikation mit Noradrenalin und Dobutamin bzw. Noradrenalin und Vasopressin verwendet. 3 von 23 Patienten (13\%) hatten eine Kombination aus Noradrenalin und Adrenalin erhalten. Bei 19 von 42 Patienten $(45,2 \%)$ ließ sich die Katecholamintherapie anhand der Unterlagen nicht eruieren. 


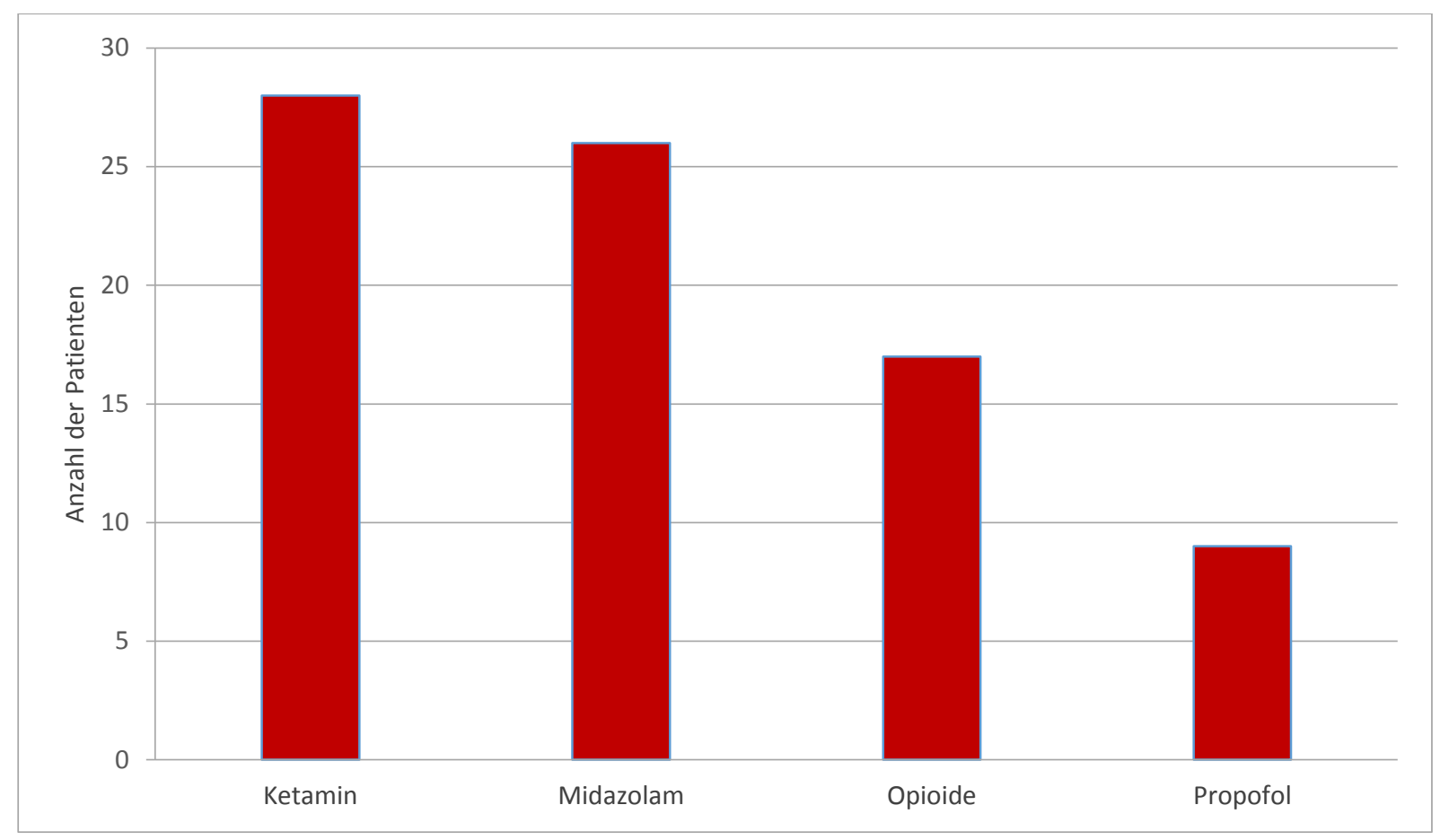

Abbildung 5: Verwendete Pharmaka zur Sedierung/Anästhesie während des Intensivaufenthaltes bei Patienten mit ischämischer Cholangiopathie der UMG (04/2007-05/2015), (Kollektiv: n=32 von 42 Patienten)

\subsubsection{Antiinfektiva während des Intensivaufenthaltes}

Zur antibiotischen, antimykotischen und antiviralen Therapie fanden sich Angaben bei 42 von 51 Patienten $(82,3 \%)$ des Gesamtkollektivs.

Bei den Antibiotika war die am häufigsten applizierte Substanzgruppe Penicilline (29 von 42 Patienten, 69\%). Hierbei wurden bei 21 von 29 Patienten $(72,4 \%)$ Piperacillin und Sulbactam bzw. Piperacillin und Combactam verabreicht. Bei 4 von 29 Patienten $(13,9 \%)$ wurde Ampicillin mit Sulbactam verabreicht, bei 2 von 29 Patienten (6,9\%) Flucloxacillin. 1 von 29 Patienten $(3,4 \%)$ hatte eine Therapie mit Amoxicillin und Clavulansäure sowie ein weiterer von 29 Patienten $(3,4 \%)$ Ampicillin als Monotherapie erhalten.

Als am zweithäufigsten verabreichte antibiotische Substanzgruppe imponierten die Carbapeneme (Meropenem und Imipenem), die bei 24 von 42 Patienten $(57,1 \%)$ eingesetzt worden waren. Hierbei wurde bei 16 von 24 Patienten (66,6\%) Meropenem verabreicht.

Am dritthäufigsten wurden Cephalosporine bei 22 von 42 Patienten (52,3\%) appliziert. Hierbei wurden mit Cefotaxim 8 von 22 Patienten (36,3\%) sowie mit Ceftriaxon 6 von 22 Patienten $(27,2 \%)$ therapiert.

Gyrasehemmer (Chinolone) kamen bei 18 von 42 Patienten (42,8\%) zum Einsatz, darunter am häufigsten Ciprofloxacin (12 von 18 Patienten, 66,6\%). 
Die Oxazolidinone mit Linezolid als Hauptvertreter dieser Gruppe wurden bei 16 von 42 Patienten (38\%) verwendet.

Mit Makroliden wurden 8 der 42 Patienten (19\%) therapiert. Hierbei wurde bei 7 von 8 Patienten Clarithromycin (87,5\%) eingesetzt.

Weiterhin erhielten 5 von 42 Patienten $(11,9 \%)$ eine antibiotische Therapie mit dem Glykopeptid Vancomycin. Bei 5 von 42 Patienten (11,9\%) wurde das Nitroimidazol Antibiotikum Metronidazol appliziert. 4 von 42 Patienten (9,5\%) erhielten eine Behandlung mit Aminoglykosiden, drei davon mit Tobramycin und ein Patient mit Gentamicin.

Bei der Auswertung der Patientendaten bezüglich der antibiotischen Therapie zeigte sich, dass häufig mehrere Antibiotika während eines Intensivaufenthaltes verabreicht worden waren. Nur bei 6 von 42 Patienten (14,2\%) war eine antibiotische Monotherapie eingesetzt worden. Die Mehrheit der Patienten, nämlich 12 von 42 Patienten (28,5\%), hatte zwei Antibiotika erhalten. Bei 8 von 42 Patienten (19\%) waren drei Antibiotika verabreicht worden. 7 von 42 Patienten (16,6\%) erhielten vier Antibiotika, 2 von 42 Patienten (4,7\%) erhielten fünf Antibiotika und 3 von 42 Patienten (7,1\%) erhielten sechs Antibiotika. Bei 3 von 42 Patienten $(7,1 \%)$ waren es sieben Antibiotikasubstanzen. In einem einzigen Fall (1 von 42 Patienten, 2,3\%) wurden insgesamt acht Antibiotika während des Intensivaufenthaltes verabreicht.

Eine antimykotische Therapie war bei 7 Patienten von 42 Patienten (16,6\%) notwendig. Darunter war Caspofungin die am häufigsten eingesetzte Substanz (3 von 7 Patienten, $42,8 \%)$.

Eine antivirale Therapie war bei 6 von 42 Patienten (14,2\%) indiziert. Hierbei erhielten 2 von 6 Patienten (33,3\%) Aciclovir, 2 von 6 Patienten (33,3\%) Oseltamivir. Eine Kombination aus Ganciclovir und Vanciclovir wurde bei je 1 von 6 Patienten $(16,6 \%)$ verabreicht.

Zusammenfassend sind die Ergebnisse der antiinfektiösen Therapie in Abbildung 6 dargestellt. 


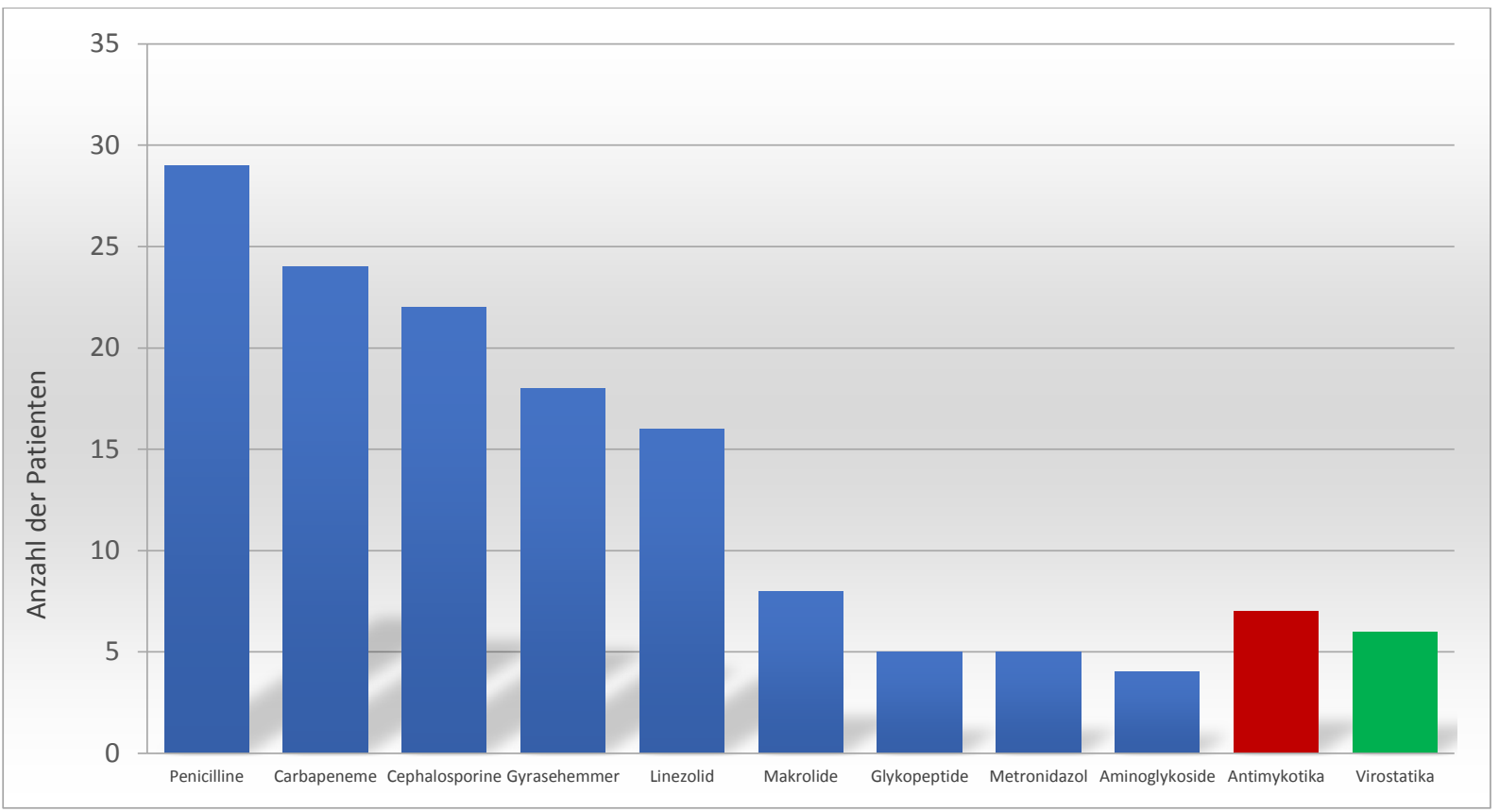

Abbildung 6: Antibiotische/antimykotische/antivirale Therapie während des Intensivaufenthaltes bei Patienten mit ischämischer Cholangiopathie der UMG (04/2007-05/2015), (Kollektiv: $n=42$ von 51 Patienten)

\subsubsection{Weitere Medikationen während des Intensivaufenthaltes}

Die Analyse der Daten ergab noch weitere Pharmaka, die jedoch nur relativ selten eingesetzt wurden. Darunter waren Amiodaron bei 8 von 42 Patienten (19\%), Mannitol bei 5 von 42 Patienten (11,9\%), Antikonvulsiva (Levitiracetam, Valproinsäure und Lamotrigin) bei 4 von 42 Patienten (9,5\%) und intravenöses Hydrocortison bei 3 von 42 Patienten (7,1\%). 2 von 42 Patienten $(4,7 \%)$ erhielten Immunglobuline und Mestinon. Je 1 von 42 Patienten (2,3\%) erhielt Mesalazin bzw. Azathioprin.

\subsubsection{Laborparameter während des Intensivaufenthaltes}

Bei 42 von insgesamt 51 Patienten $(88,2 \%)$ wurde der Verlauf verschiedener Laborparameter während des intensivmedizinischen Aufenthaltes in den ersten 42 Tagen erfasst.

Hierbei wurden folgende Laborparameter eruiert: Leberwerte (AST, ALT), Cholestasewerte (y-GT, AP, Gesamtbilirubin), Hämoglobin, Leukozyten, Quick-Wert und Kreatinin.

Albumin als Parameter der Lebersynthesefunktion konnte nicht verfolgt werden, da seine Bestimmung während des Intensivaufenthaltes nicht regelmäßig erfolgte. Dasselbe galt für das C-reaktive Protein (CRP) bzw. Procalcitonin, die ergänzend zu den Leukozyten nur unregelmäßig bestimmt wurden. 
Im Folgenden erfolgt die Darstellung der Laborparameter als Medianwert der ausgewerteten Patienten im täglichen Zeitverlauf ab dem Initialereignis.

\subsubsection{Aspartat-Aminotransferase (AST)}

Der Referenzbereich für die AST im Plasma liegt bei $\leq 35 \mathrm{U} / 1$ bei Männern und $\leq 31 \mathrm{U} / 1$ bei Frauen. Während der ersten drei Tage des Intensivaufenthaltes waren die AST-Medianwerte nahezu im Normbereich mit Werten zwischen 40,5 U/l und 31 U/l. Am vierten Tag nach Aufnahme auf Intensivstation war eine Erhöhung auf 90 U/l feststellbar. AST erreichte am 8. Tag den maximalen Anstieg im Median mit 249 U/l (Abbildung 7).

Eine wochenweise Beurteilung der Werte zeigte, dass die AST-Werte sich im Wochenmedian der ersten Woche mit 55,5 U/1 nahezu im Normbereich befanden. In der zweiten Woche stieg die AST auf einen Wert von 141,5 U/l. Der erhöhte AST-Medianwert blieb auch in den Wochen 3-6 nachweisbar mit Werten bis maximal $187 \mathrm{U} / 1$ in der sechsten Woche (Tabelle 2 und Abbildung 12).

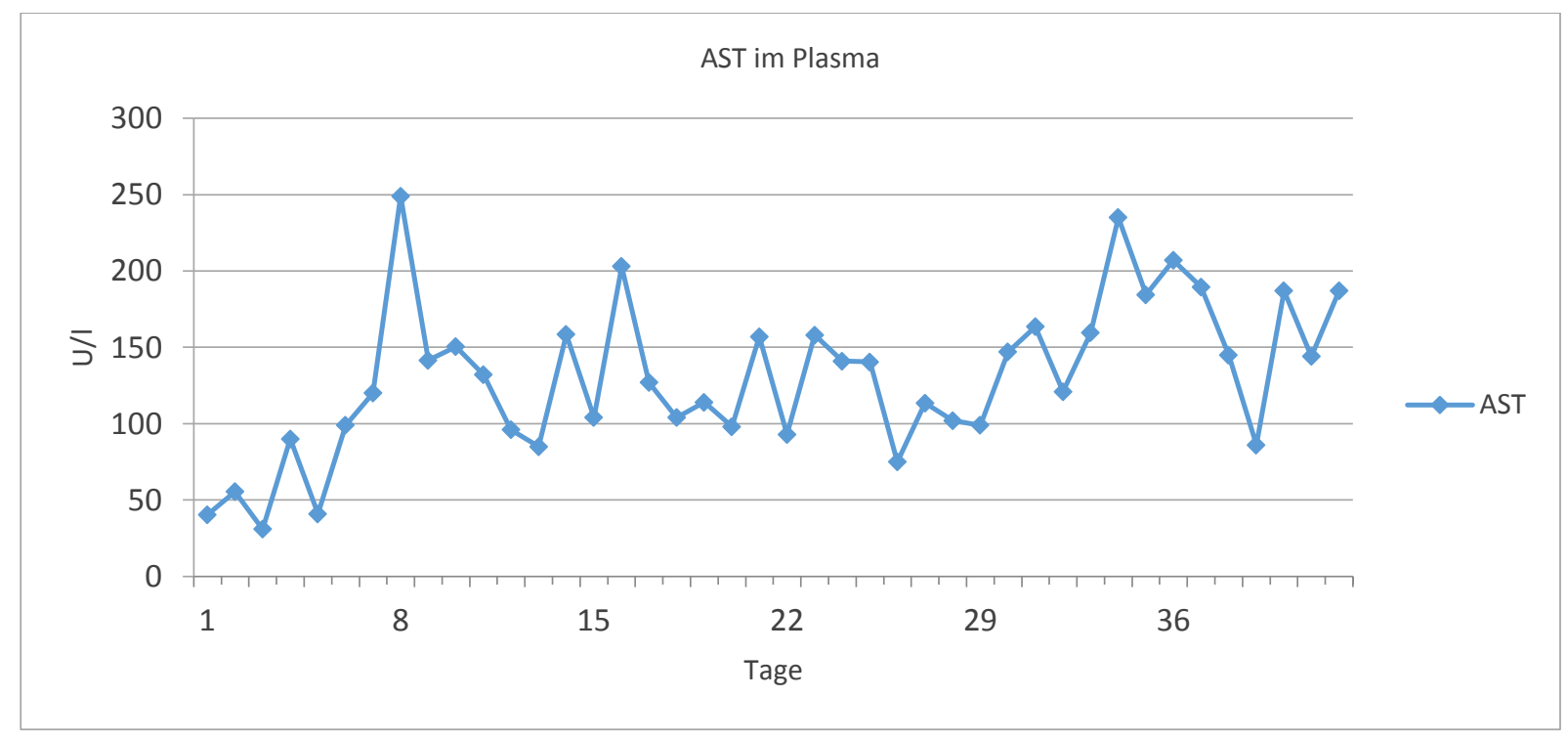

Abbildung 7: Verlauf der AST-Medianwerte während des Intensivaufenthaltes von Patienten mit ischämischer Cholangiopathie der UMG (04/2007-05/2015) über 42 Tage, (Kollektiv: $\mathrm{n}=42$ von 51 Patienten), Referenzbereich: $\leq 35$ U/1 (m); $\leq 31 \mathrm{U} / 1$ (w) 


\subsubsection{Alanin-Aminotransferase (ALT)}

Der Referenzbereich für die ALT im Plasma liegt bei $\leq 45$ U/l bei Männern und $\leq 34$ U/l bei Frauen. Die ALT-Medianwerte lagen während der 1. Woche im Referenzbereich. Ab der 2. Woche bis anschließend zum 19. Tag war ein kontinuierlicher Anstieg der Tagesmedianwerte $\mathrm{zu}$ verzeichnen. Im weiteren Verlauf zeigten sich die Tagesmediane fluktuierend mit einem zweiten Maximum von 337 U/L am 35. Tag (Abbildung 8).

Die Wochenmediane (Tabelle 2) zeigten mit einem Wert von 26,5 U/l einen normalen ALTWochenmedianwert in der ersten Woche, daraufhin jedoch mit 106 U/1 eine Erhöhung in der 2. Wochen und mit einem Wert von 233 U/1 einen Maximum in der 3. Woche.

In der 4. Woche waren die Wochenmedianwerte mit 169 U/l rückläufig und in der 5. Woche zeigte sich jedoch ein erneuter Anstieg der ALT mit einem Wochenmedian von $217 \mathrm{U} / 1$ (Tabelle 2 und Abbildung 12).

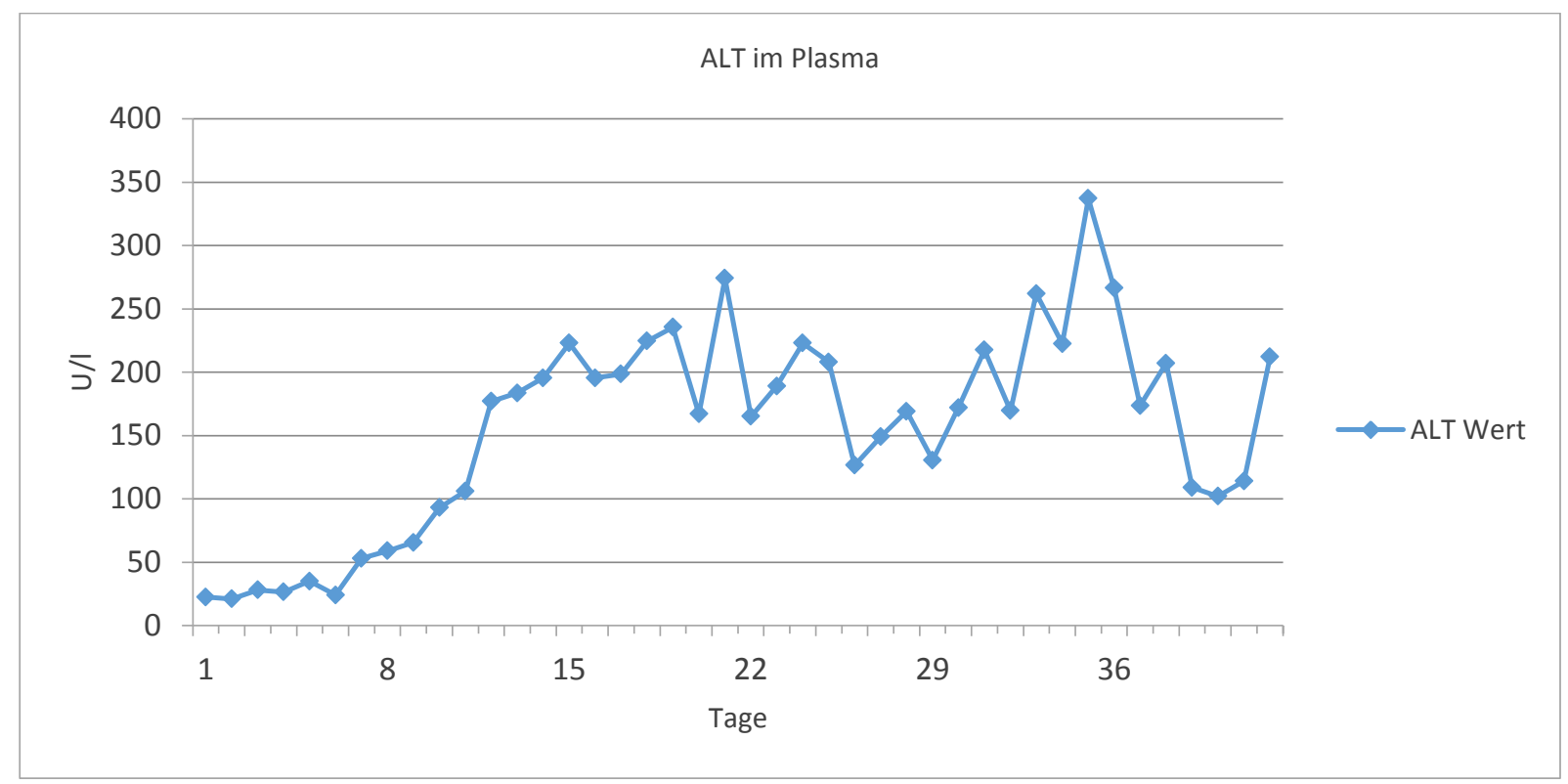

Abbildung 8: Verlauf der ALT-Medianwerte während des Intensivaufenthaltes von Patienten mit ischämischer Cholangiopathie der UMG (04/2007-05/2015) über 42 Tage, (Kollektiv:n=42 von 51 Patienten),

Referenzbereich: $\leq 45 \mathrm{U} / 1(\mathrm{~m}) ; \leq 34 \mathrm{U} / \mathrm{l}(\mathrm{w})$

\subsubsection{Gamma-Glutamyl-Transferase ( $\gamma-G T)$}

Die Referenzwerte für die y-GT im Plasma liegen bei 12-64 U/1 bei Männern und 9-36 U/l bei Frauen. Die $\gamma_{-}$GT-Werte stellten sich in den ersten 5 Tagen nach dem Initialereignis mit im 
Tagesmedian gemessenen Werten von $\leq 35$ Ul als normwertig dar. Der maximale Tagesmedianwert wurde am Tag 22 mit 1639,5 U/l erreicht. (Abbildung 9).

Der Vergleich der y-GT Wochenmedianwerte (Tabelle 2 und Abbildung 12) für die erste Woche zeigte mit $32 \mathrm{U} / \mathrm{l}$ normale Werte. Ab der 2. Woche erfolgte ein drastischer Anstieg auf einen Wert von 562 U/l. In der der 3.Woche wurde ein Maximalwert von 1254 U/l erreicht. Im weiteren Verlauf erfolgte ein Rückgang der y-GT Wochenmedianwerte, sie blieben jedoch bei einem Wert von 745 U/L weiterhin stark erhöht.

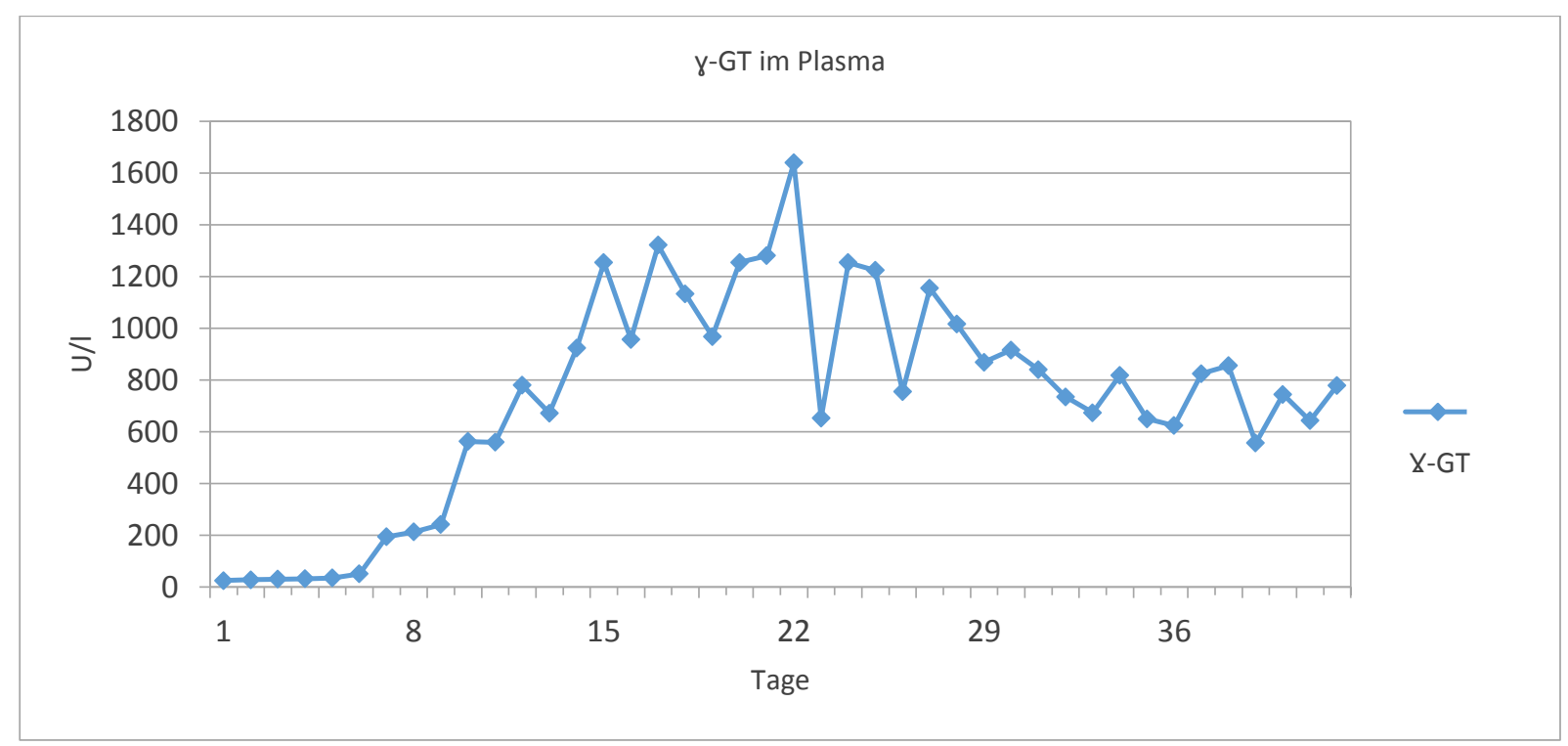

Abbildung 9: Verlauf der $\mathrm{\gamma}$-GT-Medianwerte während des Intensivaufenthaltes von Patienten mit ischämischer Cholangiopathie der UMG (04/2007-05/2015) über 42 Tage, (Kollektiv: $\mathrm{n}=42$ von 51 Patienten), Referenzbereich: 12-64 U/l (m); 9-36 U/l (w)

\subsubsection{Alkalische Phosphatase (AP)}

Der Referenzbereich für die AP im Plasma liegt bei Frauen und Männern bei 40-150 U/1.

Die Tagesmedianwerte der AP zeigten sich in den ersten 8 Tagen nach dem Initialereignis mit Werten $\leq 131,5 \mathrm{U} / 1$ als normwertig. Ab dem 9. Tag stellte sich ein Anstieg auf $162 \mathrm{U} / \mathrm{im}$ Tagesmedian dar. Dieser Trend setzte sich bis zum Ende der Beobachtungsphase mit einem maximalen Tagesmedian von 1383 U/1 am Tag 42 fort (Abbildung 10).

Der Vergleich der Wochenmediane (Tabelle 2 und Abbildung 12) zeigte einen stetigen Anstieg der AP Wochenmediane bis zu einem Maximalwert von 955 U/L in der 6. Woche. 


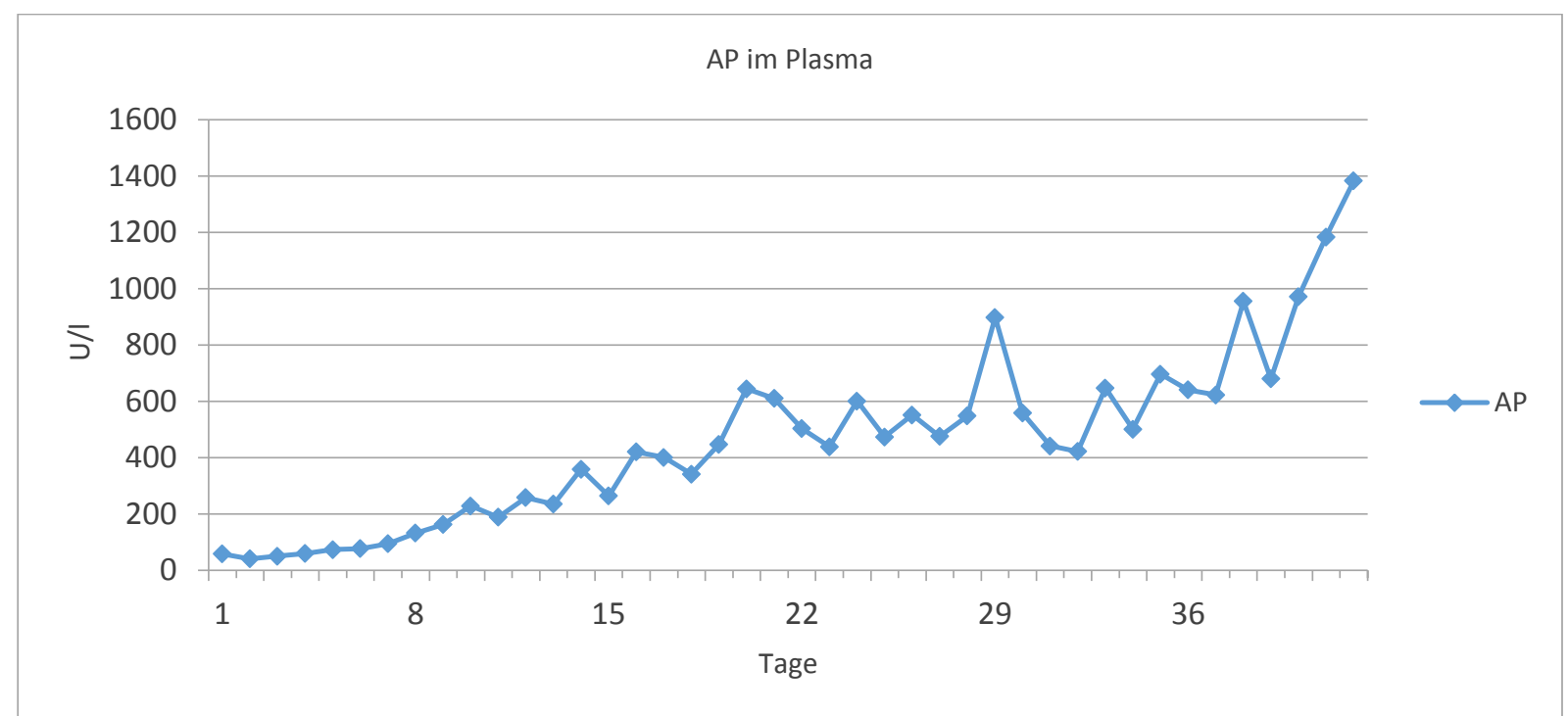

Abbildung 10: Verlauf der AP-Medianwerte während des Intensivaufenthaltes von Patienten mit ischämischer Cholangiopathie der UMG (04/2007-05/2015) über 42 Tage, (Kollektiv: $\mathrm{n}=42$ von 51 Patienten), Referenzbereich: 40-150 U/l (m)/(w)

\subsubsection{Gesamtbilirubin}

Der Referenzwert für das Gesamtbilirubin im Plasma liegt bei Frauen und Männern bei $\leq 1,2$ mg/dl. Die Tagesmediane des Gesamtbilirubins in den ersten 15 Tagen zeigten sich mit Werten von maximal $\leq 1,4 \mathrm{mg} / \mathrm{dl}$ noch grenzwertig normal. Ab der 2 . Woche zeigte sich ein fluktuierender Verlauf der Tagesmediane mit einem ersten Gipfel der Tagesmediane am Tag 25 mit einem Wert von 10,2 mg/dl. Der maximal erhöhte Tagesmedian zeigte sich am Tag 36 mit einemWert von 11,4 mg/dl (Abbildung 11).

In der tabellarischen Darstellung der Gesamtbilirubin-Wochenmediane (Tabelle 2 und Abbildung 12) zeigte sich ein kontinuierlicher Anstieg bis zum Ende der Beobachtungsphase mit einem Maximumwert von 7,45 mg/dl in der 6. Woche. 


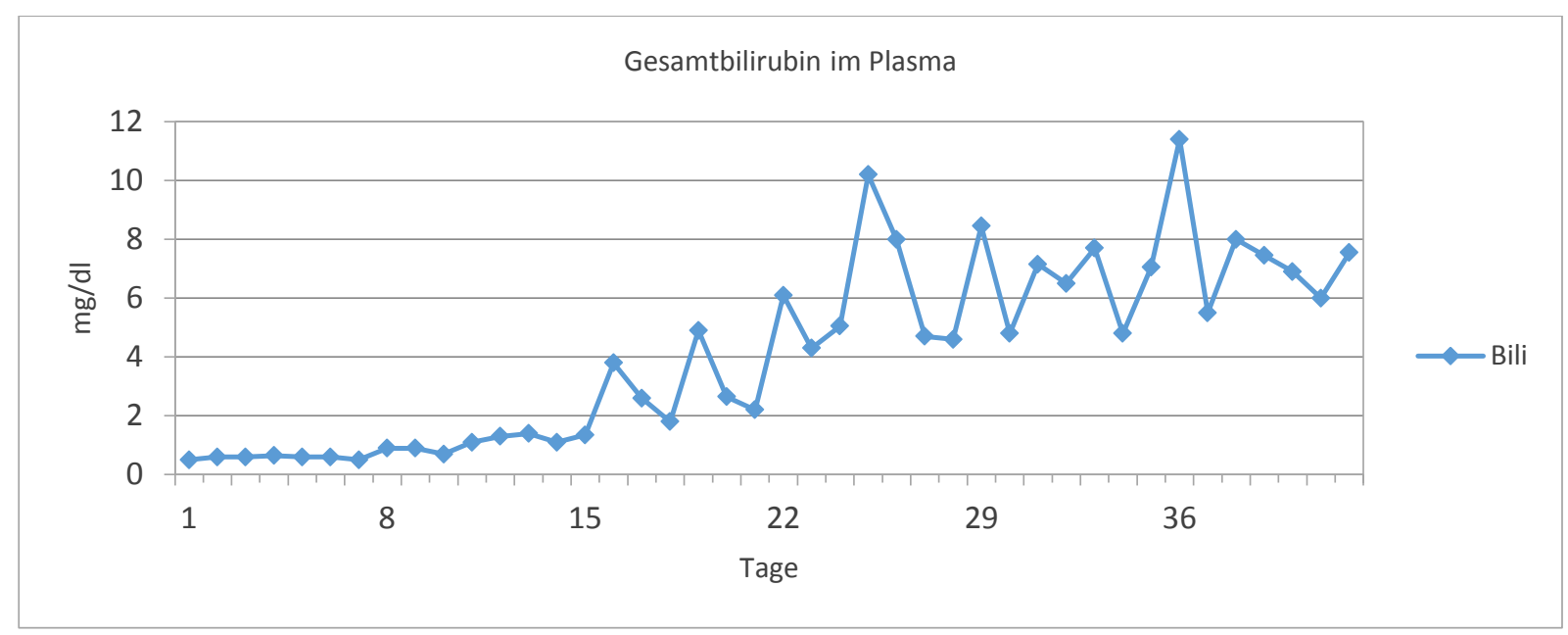

Abbildung 11: Verlauf der Gesamtbilirubin-Medianwerte während des Intensivaufenthaltes von Patienten mit ischämischer Cholangiopathie der UMG (04/200705/2015) über 42 Tage, (Kollektiv: $\mathrm{n}=42$ von 51 Patienten),

Referenzbereich: $\leq 1,2 \mathrm{mg} / \mathrm{dl}(\mathrm{m}) /(\mathrm{w})$

Bei der Betrachtung der Wochenmediane der Transaminasen und Cholestasewerte vom Ereignistag an (Tag 1) für insgesamt sechs Wochen zeigte sich, dass in der ersten Woche alle o.g. Parameter normwertig bzw. grenzwertig normal (AST) waren. Bereits in der zweiten Woche stiegen die Transaminasen und Cholestasewerte an.

Insgesamt stiegen jedoch die Transaminasenwerte in geringerem Maße an. So erreichte die AST mit 187 U/l einen maximalen Wochenmedian in Woche 6, entsprechend einer ca. 3Fachen Erhöhung verglichen mit dem Ausgangswert in Woche 1, bzw. einer ca. 5-Fachen Erhöhung gegenüber dem Referenzwert von $\leq 35$ U/l. Die ALT erreichte einen maximalen Wochenmedian in der dritten Woche mit einem Wert von 223 U/l, entsprechend einer ca. 5Fachen Erhöhung (bezogen auf den Referenzwert von $\leq 45 \mathrm{U} / \mathrm{l}$ ).

Der Anstieg der Cholestasewerte stellte sich dagegen ausgeprägter dar. Die $\gamma^{-G T}$ erreichte einen maximalen Wochenmedian von 1254 U/l in der dritten Woche und somit einen ca. 19Fachen Anstieg im Vergleich zum oberen Normwert von 64 U/l.

Bei der AP war ein kontinuierlicher Anstieg der Werte im Wochenmedian zu verzeichnen; in Woche 6 zeigte sich eine ca. 6-Fache Erhöhung über dem oberen Normwert von 150 U/l, bzw. eine 16-Fache Erhöhung verglichen mit dem Ausgangswert in Woche 1. Das Gesamtbilirubin zeigte ebenfalls einen kontinuierlichen Anstieg im Wochenmedian und erreichte den maximalen Wert in Woche 6 mit 7,45 mg/dl entsprechend einer 12-Fachen Erhöhung im Vergleich zum Ausgangswert in der ersten Woche bzw. eine 6-Fache Erhöhung im Vergleich zum Normwert von 1,2 mg/dl (Tabelle 2 und Abbildung 12). 
Tabelle 2: Verlauf der Transaminasen und Cholestasewerte im Wochenmedian während des Intensivaufenthaltes von Patienten mit ischämischer Cholangiopathie der UMG (04/2007-05/2015) über 42 Tage, (Kollektiv: $n=42$ von 51 Patienten)

\begin{tabular}{|c|c|c|c|c|c|c|c|}
\hline Parameter & Referenzwerte & Woche 1 & Woche 2 & Woche 3 & Woche 4 & Woche 5 & Woche 6 \\
\hline AST & $\begin{array}{l}\leq 35 \mathrm{U} / \mathrm{l}(\mathrm{m}) \\
\leq 31 \mathrm{U} / \mathrm{l}(\mathrm{w})\end{array}$ & 55,5 & 141,5 & 114 & 113,5 & 159,5 & 187 \\
\hline ALT & $\begin{array}{l}\leq 45 \mathrm{U} / \mathrm{l}(\mathrm{m}) \\
\leq 34 \mathrm{U} / \mathrm{l}(\mathrm{w})\end{array}$ & 26,5 & 106 & 223 & 169 & 217,5 & 173,5 \\
\hline$\gamma-G T$ & $\begin{array}{l}12-64 \mathrm{U} / 1(\mathrm{~m}) \\
9-36 \mathrm{U} / 1(\mathrm{w})\end{array}$ & 32 & 562,5 & 1254 & 1155 & 818 & 745 \\
\hline AP & $\begin{array}{l}40-150 \mathrm{U} / \mathrm{l}(\mathrm{m}) \\
40-150 \mathrm{U} / \mathrm{l}(\mathrm{w})\end{array}$ & 59 & 228 & 420,5 & 503 & 558 & 955 \\
\hline $\begin{array}{l}\text { Gesamt- } \\
\text { bilirubin }\end{array}$ & $\begin{array}{l}\leq 1,2 \mathrm{mg} / \mathrm{dl}(\mathrm{m}) \\
\leq 1,2 \mathrm{mg} / \mathrm{dl}(\mathrm{w})\end{array}$ & 0,6 & 1,1 & 2,6 & 5,05 & 7,05 & 7,45 \\
\hline
\end{tabular}

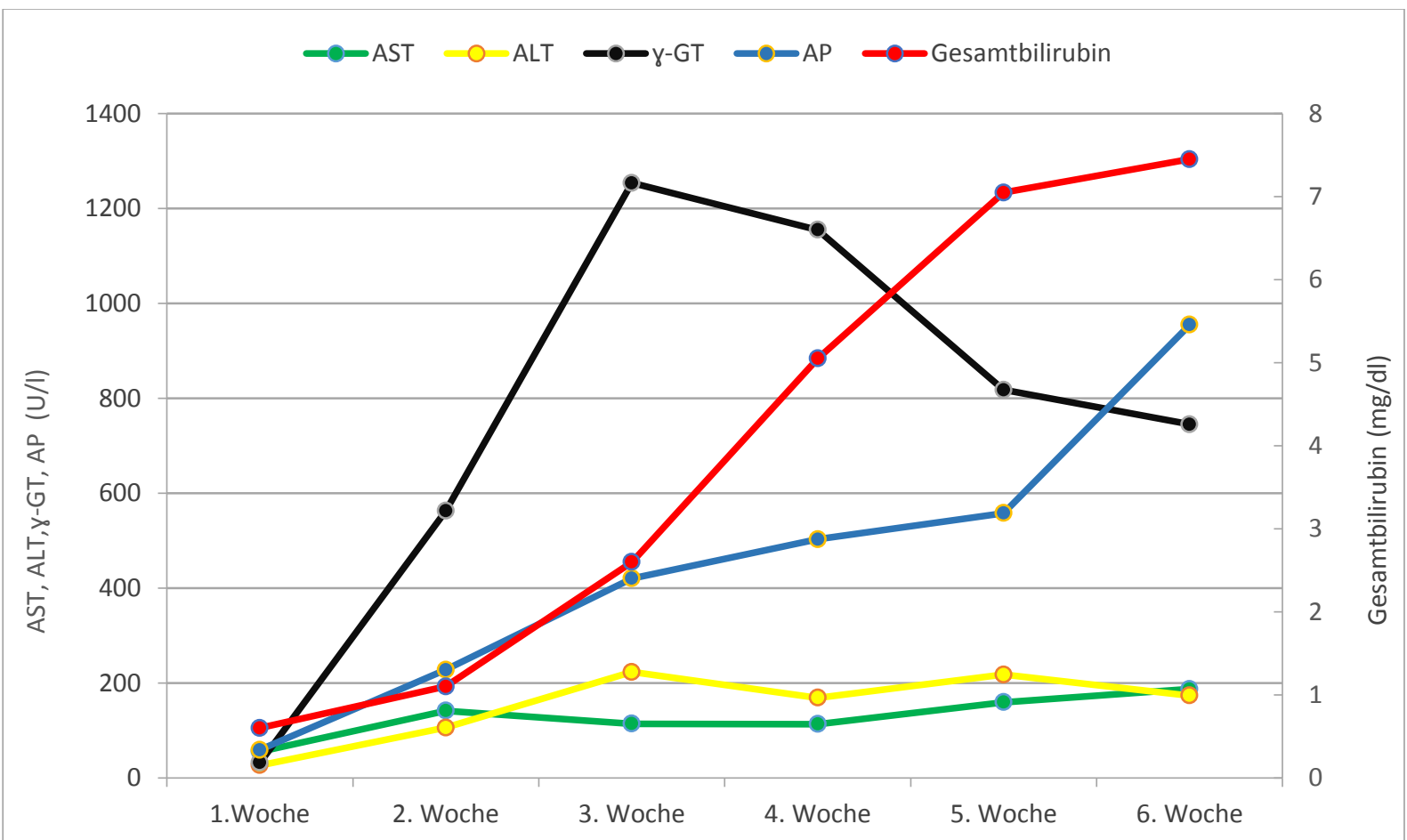

Abbildung 12: Verlauf der Transaminasen und Cholestasewerte im Wochenmedian während des Intensivaufenthaltes von Patienten mit ischämischer Cholangiopathie der UMG (04/2007-05/2015) über 42 Tage, (Kollektiv: $\mathrm{n}=42$ von 51 Patienten) 


\subsubsection{Quick-Wert}

Der Referenzbereich für den Quick-Wert liegt für beide Geschlechter bei 80-130\%. Der Quick-Wert im Plasma als möglicher Parameter der Lebersynthesestörung bei Patienten mit ischämischer Cholangiopathie zeigte sich im Median während der 42 Tage der Beobachtungsphase leicht erniedrigt bis normwertig. Der niedrigste Wert lag bei 67,5\% am Tag 40 (Abbildung 13).

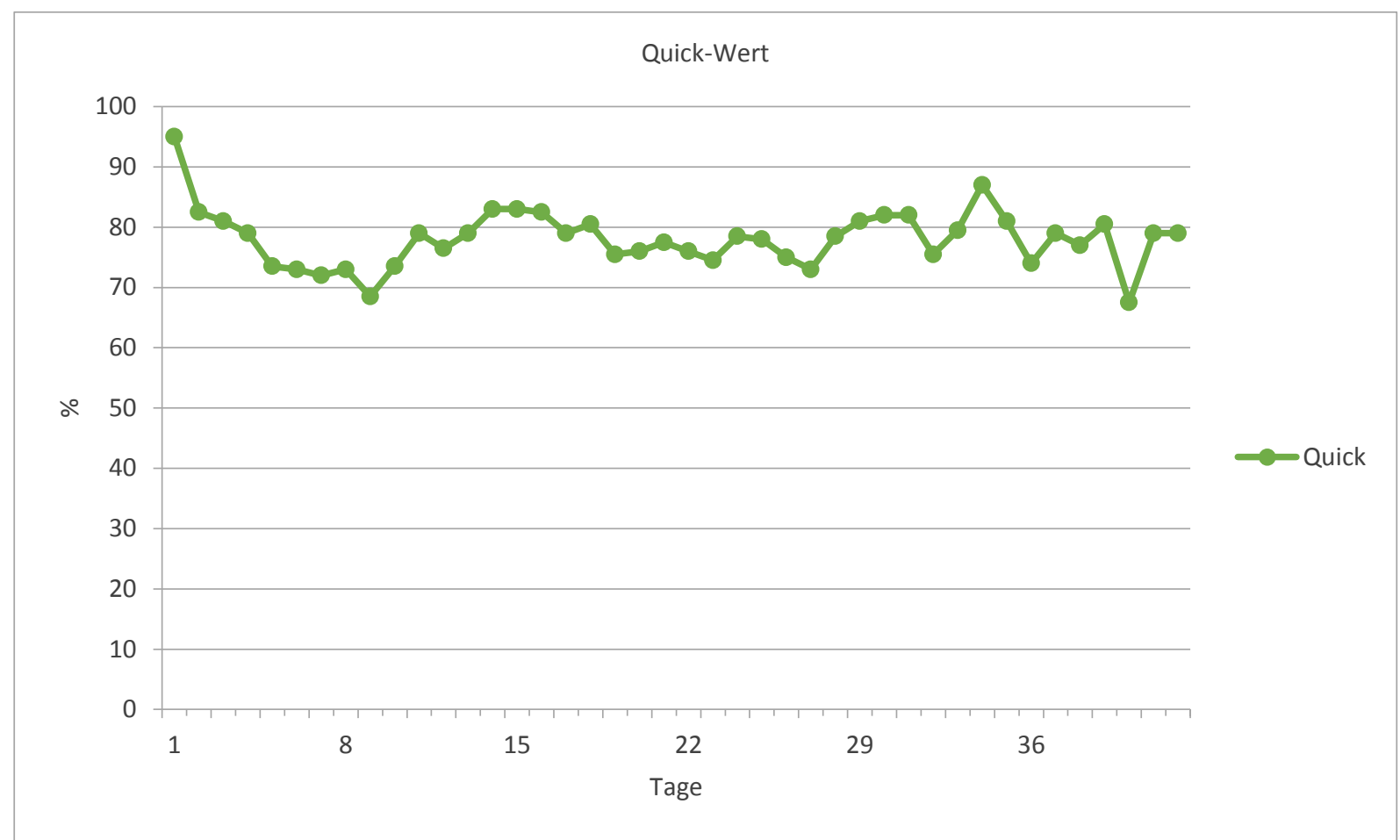

Abbildung 13: Verlauf der Quick-Wert-Medianwerte während des Intensivaufenthaltes von Patienten mit ischämischer Cholangiopathie der UMG (04/2007-05/2015) über 42 Tage, (Kollektiv: $\mathrm{n}=42$ von 51 Patienten), Referenzbereich: $80-130 \%(\mathrm{~m}) /(\mathrm{w})$

\subsubsection{Kreatinin}

Kreatinin ist bei normgewichtigen Patienten mit regulärer Muskelmasse ein Parameter für die Nierenfunktion. Der Referenzbereich beträgt 0,5-1,0 mg/dl bei Frauen und 0,7-1,2 mg/dl bei Männern.

Die Tagesmedianwerte zeigten sich bei allen Patienten in den ersten 42 Tagen normwertig bis leicht erhöht (maximaler Tagesmedianwert von 1,3 mg/dl am 38. Tag) (Abbildung 14). 


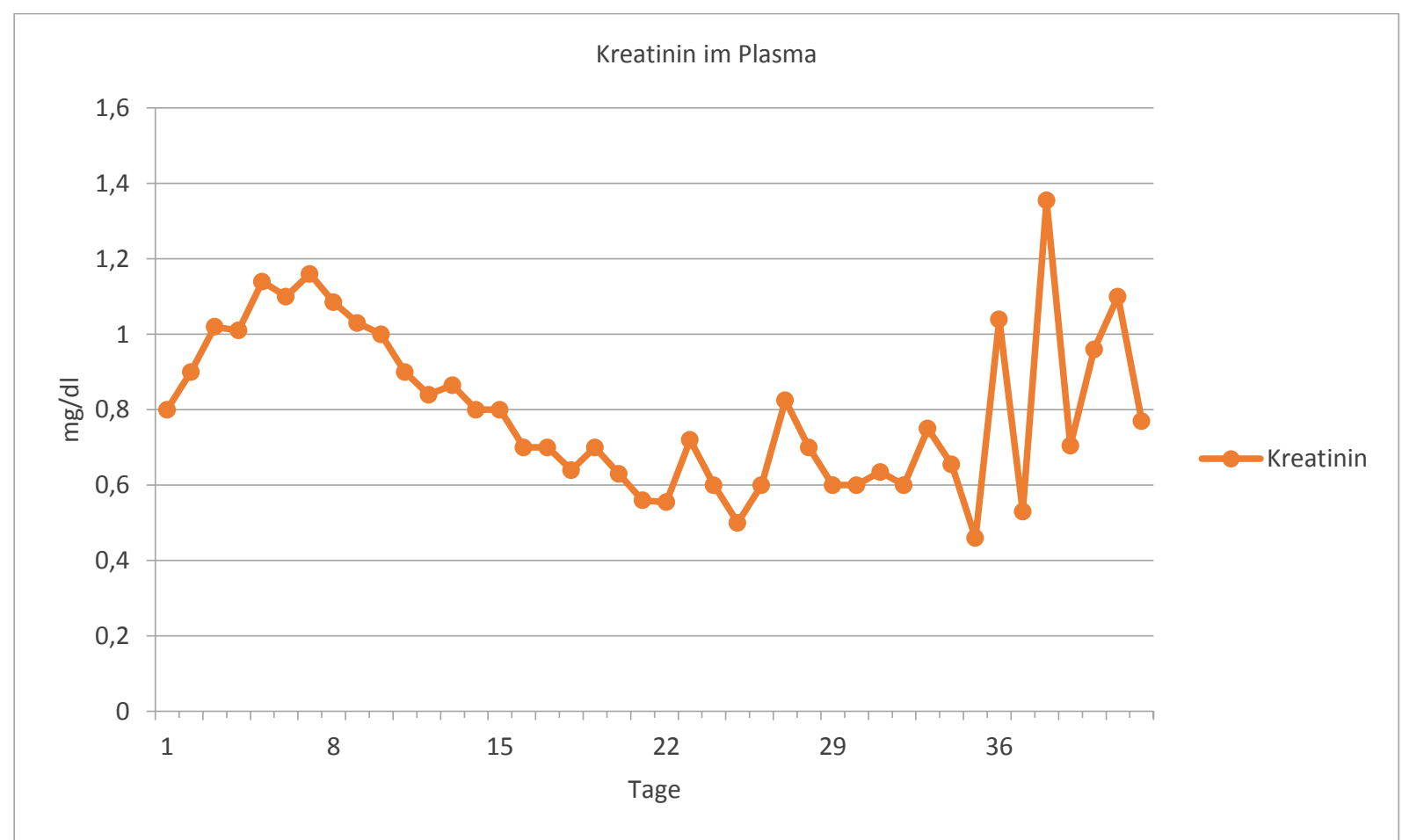

Abbildung 14: Verlauf der Kreatinin-Medianwerte während des Intensivaufenthaltes von Patienten mit ischämischer Cholangiopathie der UMG (04/2007-05/2015) über 42 Tage, (Kollektiv: $\mathrm{n}=42$ von 51 Patienten), Referenzbereich: 0,5-1,0 mg/dl (w); 0,7-1,2 mg/dl (m)

\subsubsection{Hämoglobin}

Der Referenzwert für Hämoglobin $(\mathrm{Hb})$ liegt bei Frauen bei 11,5-15 g/dl und bei Männern $13,5-17,5 \mathrm{~g} / \mathrm{dl}$.

Die Hämoglobin-Werte lagen am ersten Tag im Median bei 12,7 g/dl. Beginnend ab dem 2. Tag fielen die Werte kontinuierlich bis zum 7. Tag. Ab dem 8. Tag sowie auch im weiteren Verlauf während des Beobachtungszeitraums zeigten sich die Tagesmediane der $\mathrm{Hb}$ Werte durchgehend erniedrigt. Die Werte fluktuierten und lagen zwischen 8 bis $9 \mathrm{~g} / \mathrm{dl}$. Aufgrund dieser Werte bestand eine Anämie der intensivmedizinisch behandelten Patienten (Abbildung $15)$. 


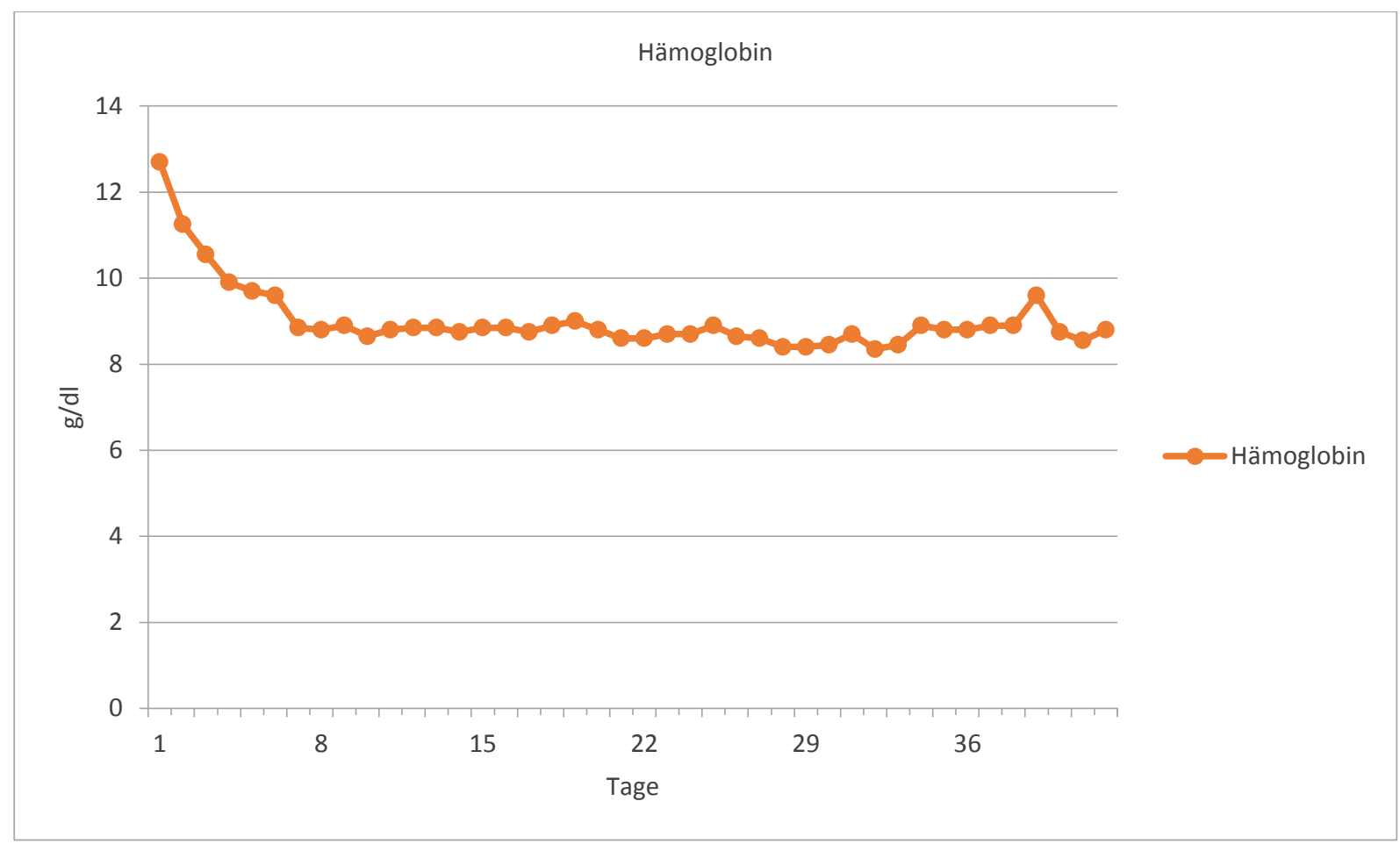

Abbildung 15: Verlauf der Hämoglobin-Medianwerte während des Intensivaufenthaltes von Patienten mit ischämischer Cholangiopathie der UMG (04/2007-05/2015) über 42 Tage, (Kollektiv: $\mathrm{n}=42$ von 51 Patienten), Referenzbereich: 11,5- $15 \mathrm{~g} / \mathrm{dl}(\mathrm{w}) ; 13,5-17,5 \mathrm{~g} / \mathrm{dl}(\mathrm{m})$

\subsubsection{Leukozyten}

Der Referenzbereich der Leukozyten liegt bei 4.000-11.000/ $\mu$ l bei beiden Geschlechtern.

Im Tagesmedian zeigten sich die Leukozytenwerte fast im gesamten Verlauf nahezu stabil (Abbildung 16).

Die Wochenmedianwerte bestätigten normwertige Leukozytenwerte in den ersten vier Wochen. In der 5-ten und 6-ten Woche zeigten sich diese mit einem Wert von 13.700/ $\mu 1$

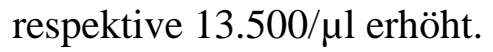




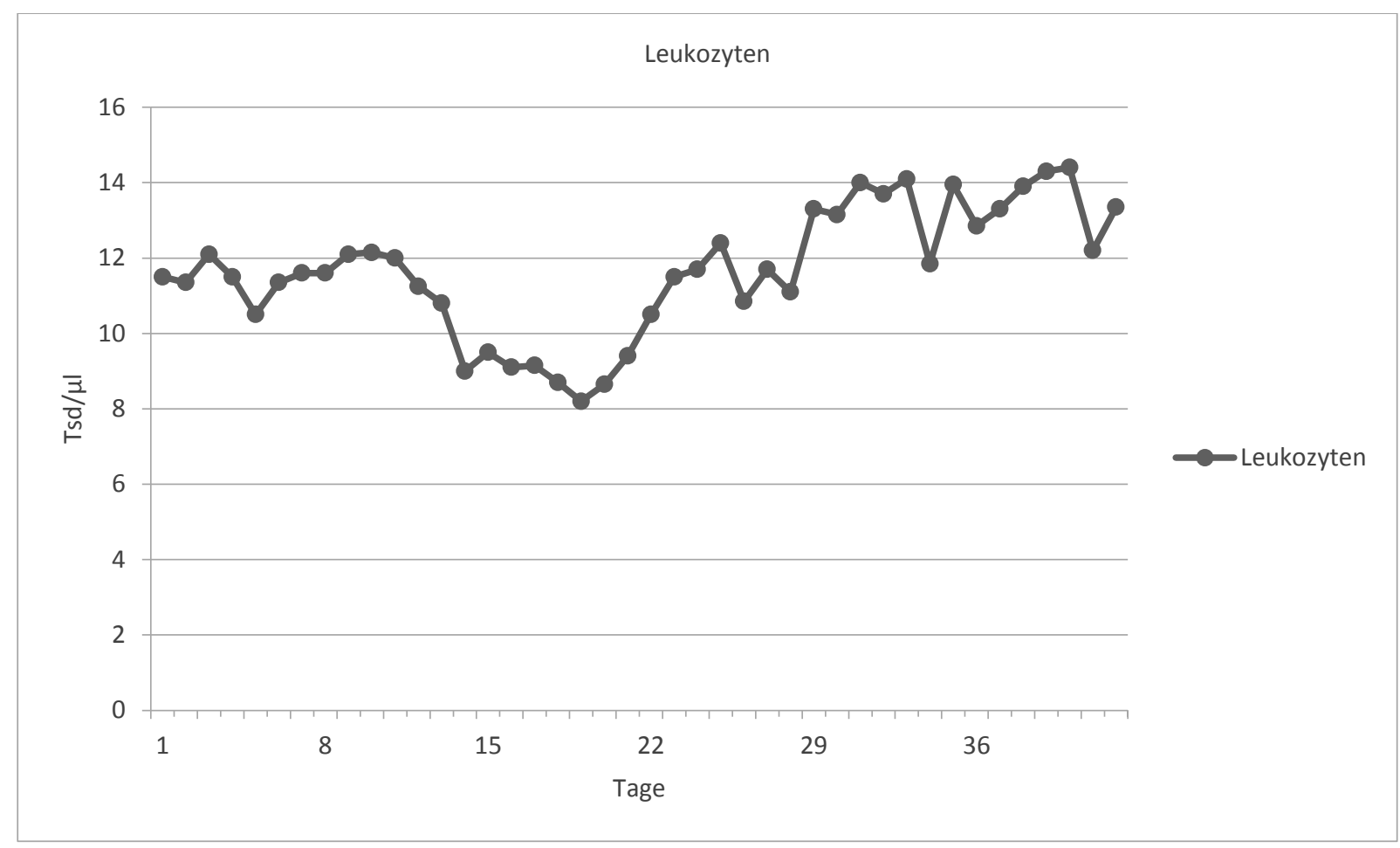

Abbildung 16: Verlauf der Leukozyten-Medianwerte während des Intensivaufenthaltes von Patienten mit ischämischer Cholangiopathie der UMG (04/2007-05/2015) über 42 Tage, (Kollektiv: $\mathrm{n}=42$ von 51 Patienten), Referenzbereich: 4.000-11.000/ $\mathrm{ll}(\mathrm{w}) /(\mathrm{m})$

\subsubsection{Arterielle Blutgasanalyse ( $\mathrm{pH}, \mathrm{pO}_{2}, \mathrm{pCO}_{2}$, Laktat)}

Eine arterielle Blutgasanalyse (BGA) während des Intensivaufenthaltes lag bei insgesamt 36 von 51 Patienten $(70,5 \%)$ vor. Der initiale Wert wurde meist auf Intensivstation oder Notaufnahme bestimmt. Bei 5 der 36 Patienten (13,8 \%) hatten die Unterlagen keine Information bezüglich der arteriellen BGA am Tag 1 (Ereignistag) ergeben. Ab dem 2. Tag lagen Werte von 36 Patienten vor.

Um danach möglichst konstante Vergleichswerte zu verwenden, wurden im weiteren Verlauf (ab Tag 2) BGA-Werte für die Auswertung verwendet, die im Intensivalltag der UMG standardmäßig jeden Morgen um 4:00 Uhr bestimmt worden.

pH-Wert

Der Referenzbereich für den arteriellen pH-Wert liegt bei Erwachsenen bei 7,35-7,45.

Der arterielle pH-Wert (Abbildung 17) war am ersten Tag des Initialereignisses bei 31 Patienten (60,7\% des Gesamtkollektivs) zu eruieren. 
Der Medianwert des ersten Tages lag bei 7,36 bei einem minimalen pH-Wert von 7,2 bei 6 von 36 Patienten (16,6\%) und einem maximalen Wert von 7,6 bei 1 von 36 der Patienten (2,7 $\%)$.

Die Betrachtung der Tagesmediane zeigte kontinuierlich einen normwertigen arteriellen $\mathrm{pH}$ Wert über die Beobachtungsphase von 30 Tagen. Es ließ sich jedoch ein Trend zu einem niedrig normalen $\mathrm{pH}$ am Anfang ( $\mathrm{pH}$ am Tag 1: 7,36) und einen hoch normwertigen $\mathrm{pH}$ am Ende der Beobachtungsphase (pH am Tag 30: 7,49) feststellen.

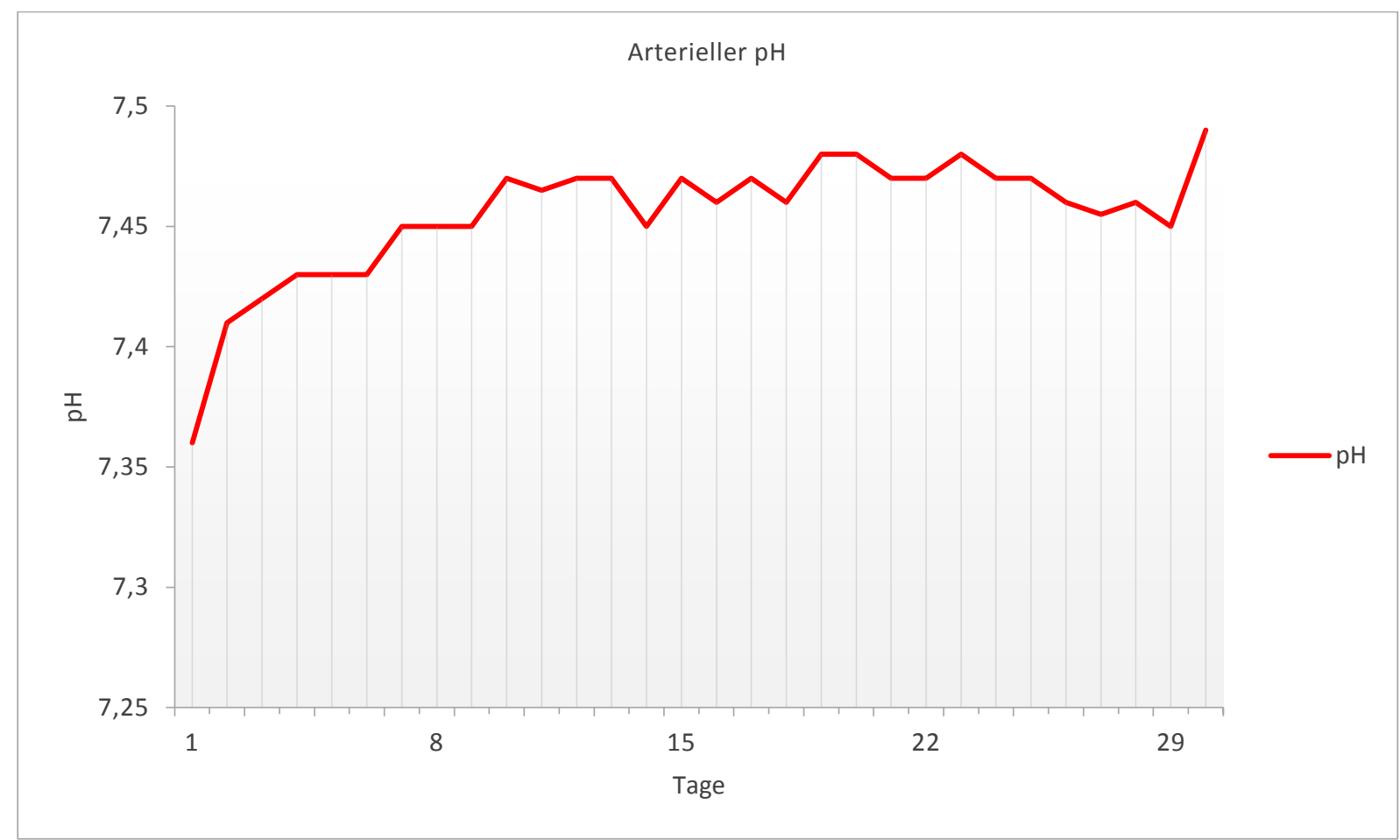

Abbildung 17: Verlauf der pH-Medianwerte während des Intensivaufenthaltes von Patienten mit ischämischer Cholangiopathie der UMG (04/2007-05/2015) über 30 Tage, (Kollektiv: $\mathrm{n}=36$ von 51 Patienten), Referenzbereich: 7,35-7,45

$\mathrm{pO}_{2}$-Wert

Der Referenzbereich für den arteriellen $\mathrm{pO}_{2}$-Wert liegt bei 65-105 mmHg. Am 1. Tag (Initialereignis) war der $\mathrm{pO}_{2}$ bei 31 Patienten (60,7 \% des Gesamtkollektivs) ermittelt worden. Dabei lag der $\mathrm{pO}_{2}$-Wert bei nur 3 von 31 Patienten (9,6 \%) unterhalb des Normwertes von 65 mmHg. Der niedrigste Wert am 1. Tag war 57 mmHg während der höchste Wert des 1 . Tages bei $600 \mathrm{mmHg}$ lag.

Die Auswertung der Tagesmediane (ab Tag 2 lagen Werte für 36 Patienten vor) im weiteren Verlauf von 30 Tagen des Intensivaufenthaltes zeigte ab dem 2. Tag durchgehend $\mathrm{pO}_{2}$ - 
Tagesmedianwerte im physiologischen Bereich. Hierbei betrug der niedrigste Wert 84,5 mmHg am Tag 19 und der höchste Wert 102 mmHg am Tag 29 (Abbildung 18).

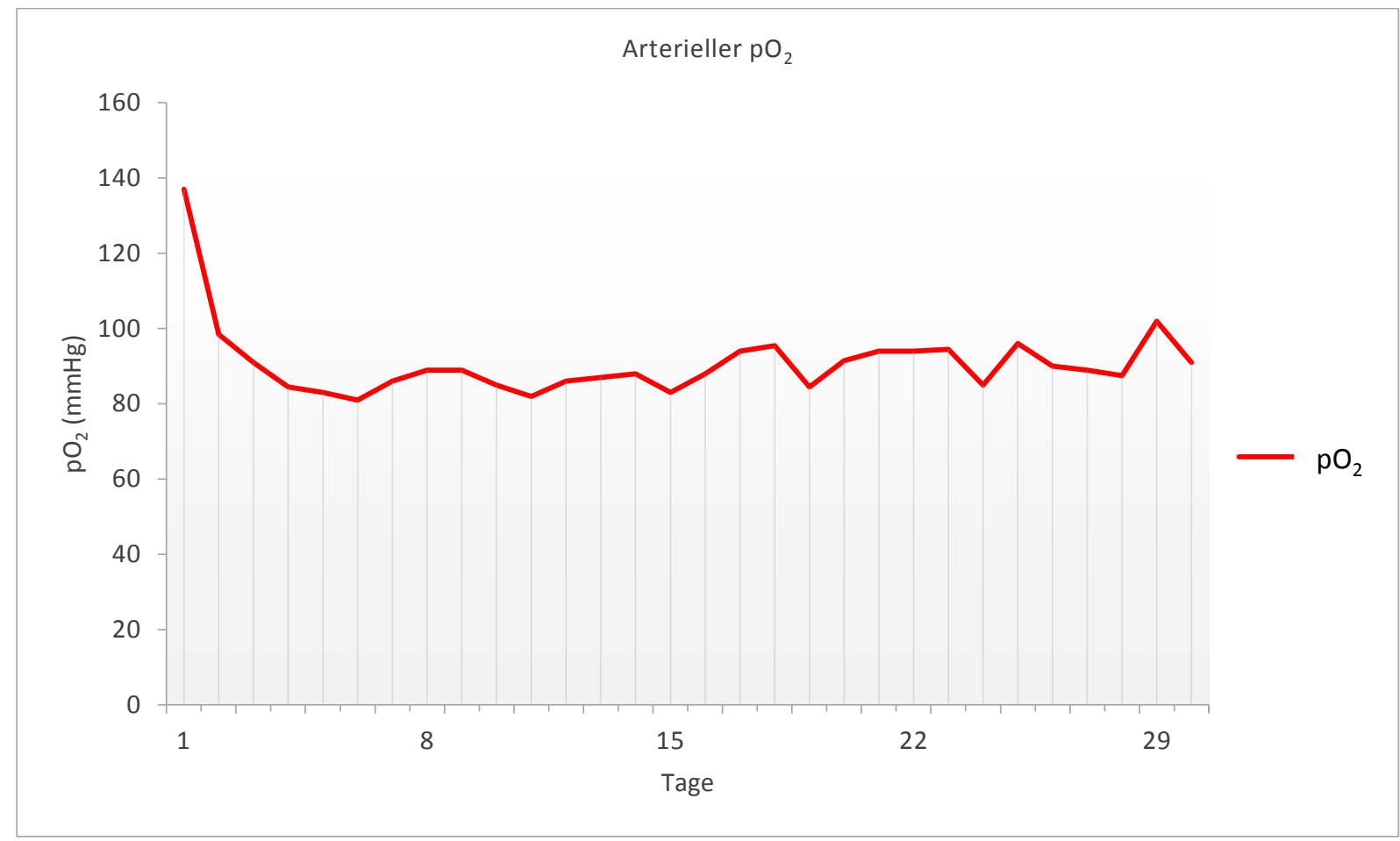

Abbildung 18: Verlauf der $\mathrm{pO}_{2}$-Medianwerte während des Intensivaufenthaltes von Patienten mit ischämischer Cholangiopathie der UMG (04/2007-05/2015) über 30 Tage, (Kollektiv: $\mathrm{n}=36$ von 51 Patienten), Referenzbereich $65-105 \mathrm{mmHg}$

$\mathrm{pCO}_{2}$-Wert

Der Referenzbereich für den arteriellen $\mathrm{pCO}_{2}$-Wert liegt bei $35-45 \mathrm{mmHg}$. Die Tagesmedianwerte des arteriellen Kohlendioxid-Partialdrucks (arterieller $\mathrm{pCO}_{2}$ ) lagen im gesamten Verlauf des Intensivaufenthaltes im Normbereich mit einem Minimum von 35 mmHg sowie einen maximalen Wert von $41 \mathrm{mmHg}$ (Abbildung 19). 


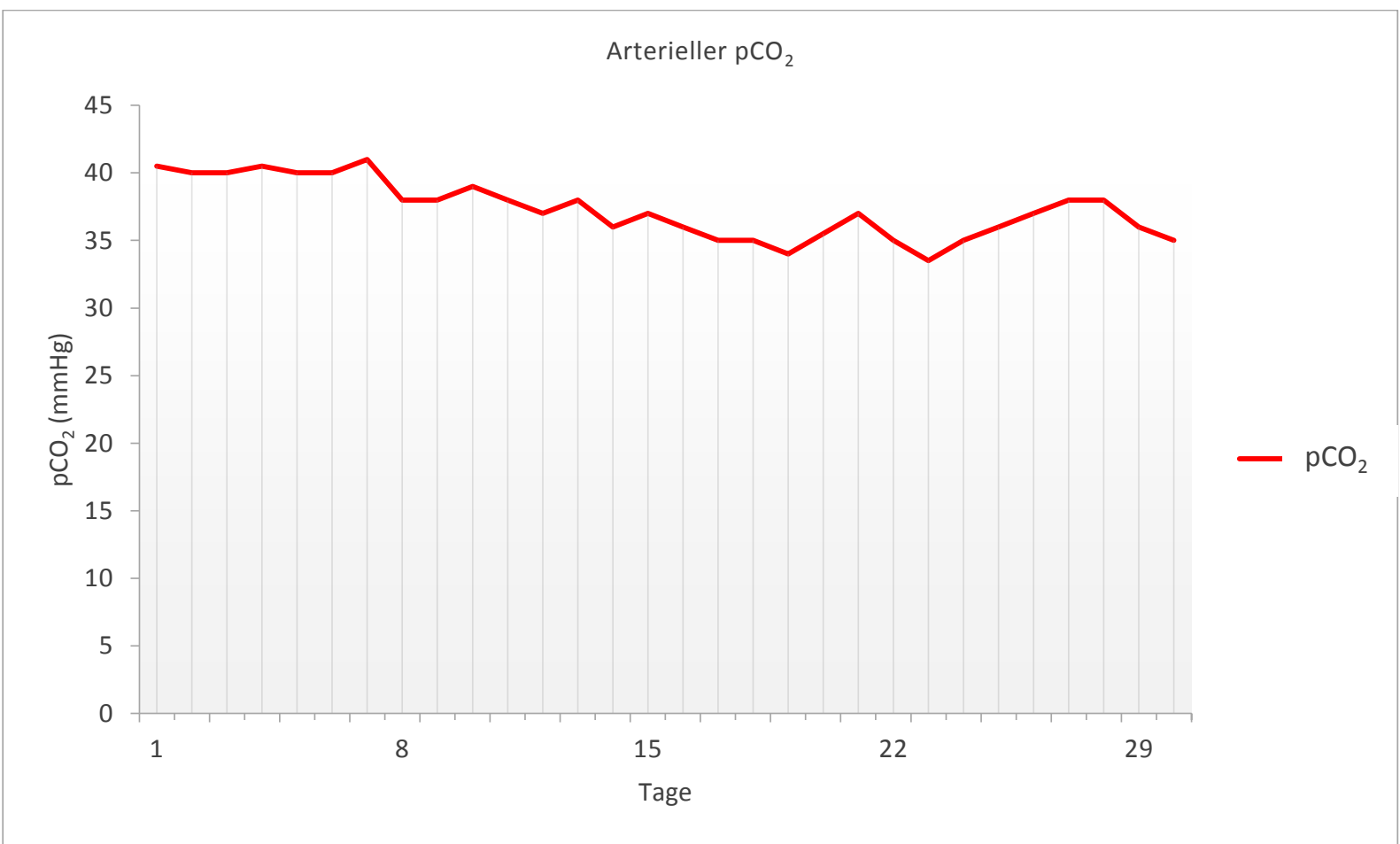

Abbildung 19: Verlauf der $\mathrm{pCO}_{2}$-Medianwerte während des Intensivaufenthaltes von Patienten mit ischämischer Cholangiopathie der UMG (04/2007-05/2015) über 30 Tage, (Kollektiv: n=36 von 51 Patienten), Referenzbereich: 34-45 $\mathrm{mmHg}$

\section{Laktat-Wert}

Der Referenzbereich für das arterielle Laktat liegt bei <1,8 mmol/l. Der Tagesmedian des arteriellen Laktat-Wertes zeigte am 1. Tag eine leichte Erhöhung (2,1 mmol/l).

Die Mehrheit der 31 Patienten $(64,5 \%)$ hatte einen Laktat-Wert am 1. Tag, der über der Norm war. Der Maximalwert betrug 5,5 mmol/l bei einem der 31 Patienten. Einen normwertigen Laktat-Wert hatten am 1. Tag nur 10 der 31 Patienten während die restlichen 20 Patienten arterielle Laktat-Werte zwischen 2-4,6 mmol/l zeigten.

Die Betrachtung der Tagesmediane zeigte ab dem Folgetag (Tag 2) bei 36 Patienten im weiteren Krankheitsverlauf durchgehend eine normwertige arterielle Laktatkonzentration (Abbildung 20). 


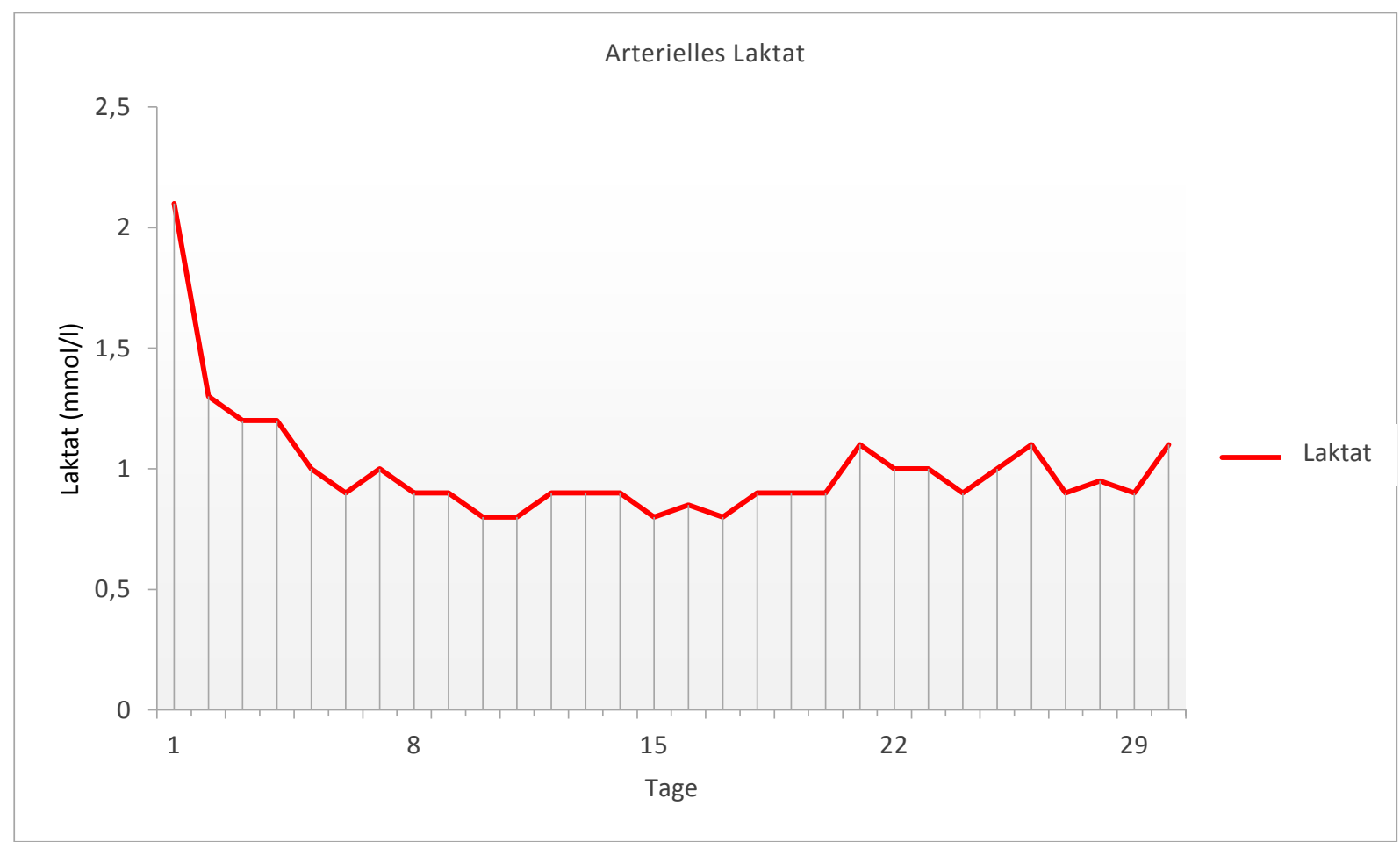

Abbildung 20: Verlauf der Laktat-Medianwerte während des Intensivaufenthaltes von Patienten mit ischämischer Cholangiopathie der UMG (04/2007-05/2015) über 30 Tage, (Kollektiv: n=36 von 51 Patienten), Referenzbereich: $<1,8$ $\mathrm{mmol} / \mathrm{l}$

\subsubsection{Laborwerte zum Zeitpunkt der Diagnosestellung mittels ERC}

Bei 42 der in der UMG primär diagnostizierten Patienten mit ischämischer Cholangiopathie (82,3\% des Gesamtkollektivs von 51 Patienten) wurden folgende Laborparameter, die maximal drei Tage vor der Untersuchung bestimmt wurden, betrachtet: Hämoglobin, Leukozyten, Quick-Wert, Transaminasen und Cholestaseparameter sowie Kreatinin.

Es zeigte sich eine deutliche Erhöhung der Cholestasewerte. Der Gesamtbilirubin-Wert lag im Median bei 7,7 mg/dl und damit über dem 6-Fachen des Normwertes von $\leq 1,2 \mathrm{mg} / \mathrm{dl}$. Der Medianwert der AP lag bei 757 U/1 und somit beim 5-Fachen des oberen Normwertes.

Der y-GT-Wert lag bei 905 U/l (16-Fache Erhöhung über dem oberen Referenzwert von 64 U/1). Die Medianwerte der Transaminasen erwiesen sich mit einem Wert von 96 U/1 für die AST bzw. 90,5 U/l für ALT als nur geringgradig erhöht.

Der Quick-Wert sowie Kreatinin lagen im Normbereich. Weiterhin waren die Leukozyten mit einem Median von 10.000/ $\mu \mathrm{l}$ im Normbereich. Hingegen war der Hb-Medianwert mit 9,5 g/dl erniedrigt und die Patienten wiesen somit eine Anämie auf. 


\subsection{Bildgebende Diagnostik vor Diagnosestellung}

Die Auswertung der bildgebenden Untersuchungen, die vor Diagnosesicherung durchgeführt worden waren, ergab, dass bei 43 von 51 Patienten $(84,3 \%)$ abdomensonographische Untersuchungen vorgenommen worden waren. Eine Computertomographie (CT) des Abdomens wurde bei 30 von 51 Patienten (58,8\%), eine endosonographische Untersuchung des oberen Gastrointestinaltraktes (EUS) wurde bei 5 von 51 Patienten $(9,8 \%)$ durchgeführt. Bei jeweils 1 von 51 Patienten (1,9\%) erfolgte eine MRT/MRCP bzw. eine PETUntersuchung (Abbildung 21).

In vielen Fällen waren einige der o.g. bildgebenden Verfahren in kurzem zeitlichem Abstand zur Diagnosesicherung mehrfach durchgeführt worden (detaillierte Beschreibung siehe unten). Um für die Auswertung vergleichbare Befunde $\mathrm{zu}$ verwenden, wurde bei den bildgebenden Verfahren stets die jeweils letzte Untersuchung gewählt, die vor der Diagnosestellung stattgefunden hatte. Dabei lag ein besonderes Augenmerk auf denjenigen Befunden der bildgebenden Diagnostik, die mit einer Erhöhung der Leber/Cholestasewerte einhergehen. In der Auswertung wurden Parameter wie Leberkontur und -größe, Leberparenchym, Lebergefäße (Vena portae, Leberarterien und Lebervenen), Raumforderungen des Lebergewebes, Gallenwege und Gallenblase einzeln analysiert.

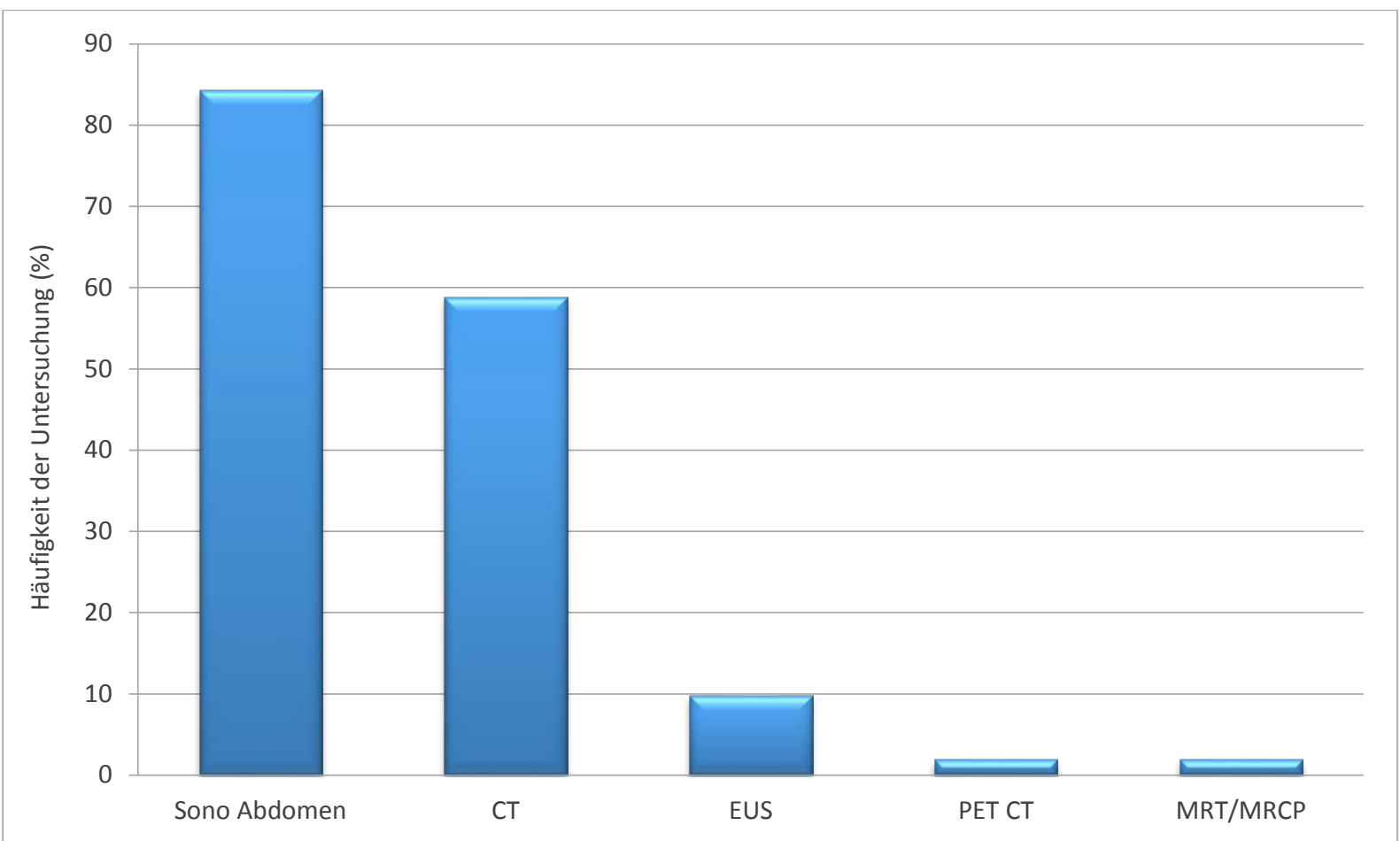

Abbildung 21: Prozentuale Verteilung der diagnostischen Bildgebung der Patienten mit ischämischer Cholangiopathie der UMG (04/2007-05/2015),

(Kollektiv: $\mathrm{n}=51$ ) 


\subsubsection{Abdomensonographie}

Bei 43 Patienten (84,3\% des Patientenkollektivs) war vor der Diagnosesicherung mittels ERC eine Ultraschalluntersuchung des Abdomens erfolgt. 23 der 43 Patienten $(53,4 \%)$ hatten nur eine einzige abdomensonographische Untersuchung. 2 von 43 Patienten $(4,6 \%)$ hatten drei Untersuchungen und bei zwei weiteren Patienten $(4,6 \%)$ waren es vier Untersuchungen. Des Weiteren fanden sich bei der Datenauswertung jeweils 1 von 43 Patienten (2,3\%) mit 4, 5, 6 und 7 Untersuchungen.

Mittels bildgebender sonographischer Diagnostik konnten folgende pathologischen Befunde festgestellt werden: Bei 19 von 43 Patienten (44,2\%) bestand eine Hepatomegalie (Messung der Leber im subkostalen Paramedianschnitt: Werte von $>14 \mathrm{~cm}$ in der Medioclavicularlinie), bei je 7 Patienten $(16,2 \%)$ war eine Steatosis hepatis bzw. eine Veränderung von Leberkontur und Leberparenchym festzustellen.

Bei 2 von 43 Patienten $(4,6 \%)$ wurden Raumforderungen der Leber beschrieben, bei 2 von 43 Patienten (4,6\%) eine Leberzirrhose in einem Fall (2,3\%) ein Leberabszess.

Bezüglich der Gallenwege war bei 14 von 43 Patienten (32,5\%) eine extrahepatische Cholestase beschrieben worden und bei 13 Patienten (30,2\%) eine intrahepatische Cholestase. Eine Choledocholithiasis bestand in 5 Fällen $(11,6 \%)$.

Bei 18 von 43 Patienten (41,9\%) war eine Cholezystolithiasis sowie Gallenblasensludge nachweisbar. Bei 3 von 43 Patienten (6,9\%) wurde eine Cholezystitis oder ein Gallenblasenhydrops vorgefunden (Abbildung 22). 


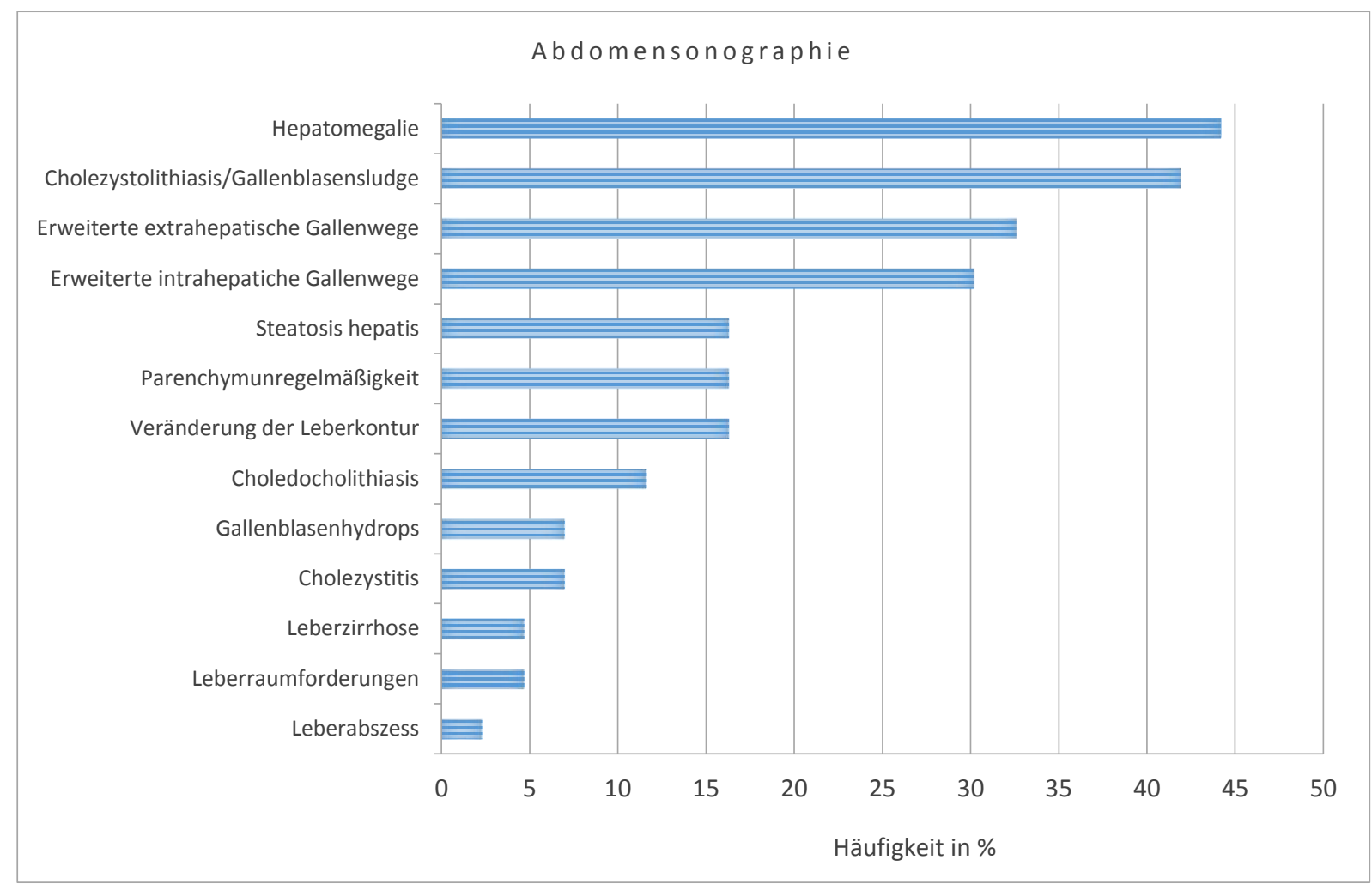

Abbildung 22: Prozentuale Verteilung pathologischer Befunde der Abdomensonographie bei Patienten mit ischämischer Cholangiopathie der UMG (04/2007-05/2015) vor Diagnosesicherung, (Kollektiv: $\mathrm{n}=43$ von 51 Patienten)

\subsubsection{Computertomographie (CT)}

Bei 30 der 51 Patienten des Gesamtkollektivs (58,8\%) waren computertomographische Untersuchungen des Abdomens durchgeführt worden (Abbildung 23). Dabei hatten 22 der 30 Patienten (66,6\%) eine einzige CT-Untersuchung, fünf Patienten (16,6\%) drei CTUntersuchungen und zwei Patienten (6,6\%) zwei CT-Untersuchungen. Ein einziger Patient (3,3\%) wies neun CT-Untersuchungen auf. Anhand der schriftlichen radiologischen Befunde wurden die Informationen über Leberstruktur, Lebergefäße und Gallenwege analysiert.

Bei 3 von 30 Patienten (10\%) war eine Hepatomegalie erwähnt. Bei 2 von 30 Patienten $(6,6 \%)$ waren Unregelmäßigkeiten des Leberparenchyms beschrieben. Leberraumforderungen waren bei 2 von 30 Patienten (6,6\%) nachweisbar. Bei je einem von 30 Patienten (3,3\%) fand sich eine Steatosis hepatis bzw. eine Veränderung der Leberkontur oder ein Leberabszess. Eine Leberzirrhose hatte CT-morphologisch in keinem Fall vorgelegen.

Hinsichtlich der Gallenwege war bei 13 von 30 Patienten (43,3\%) eine Erweiterung der intrahepatischen Gallenwege und bei 7 von 30 Patienten (23,3\%) eine Erweiterung der extrahepatischen Gallenwege dokumentiert. Bei einem Patienten (3,3\%) lag eine Choledocholithiasis vor. 
Bei 5 von 30 Patienten (16,7\%) war eine Cholezystolithiasis oder Gallenblasensludge vorhanden. Jeweils drei Patienten (10\%) hatten eine Cholezystitis oder einen Gallenblasenhydrops.

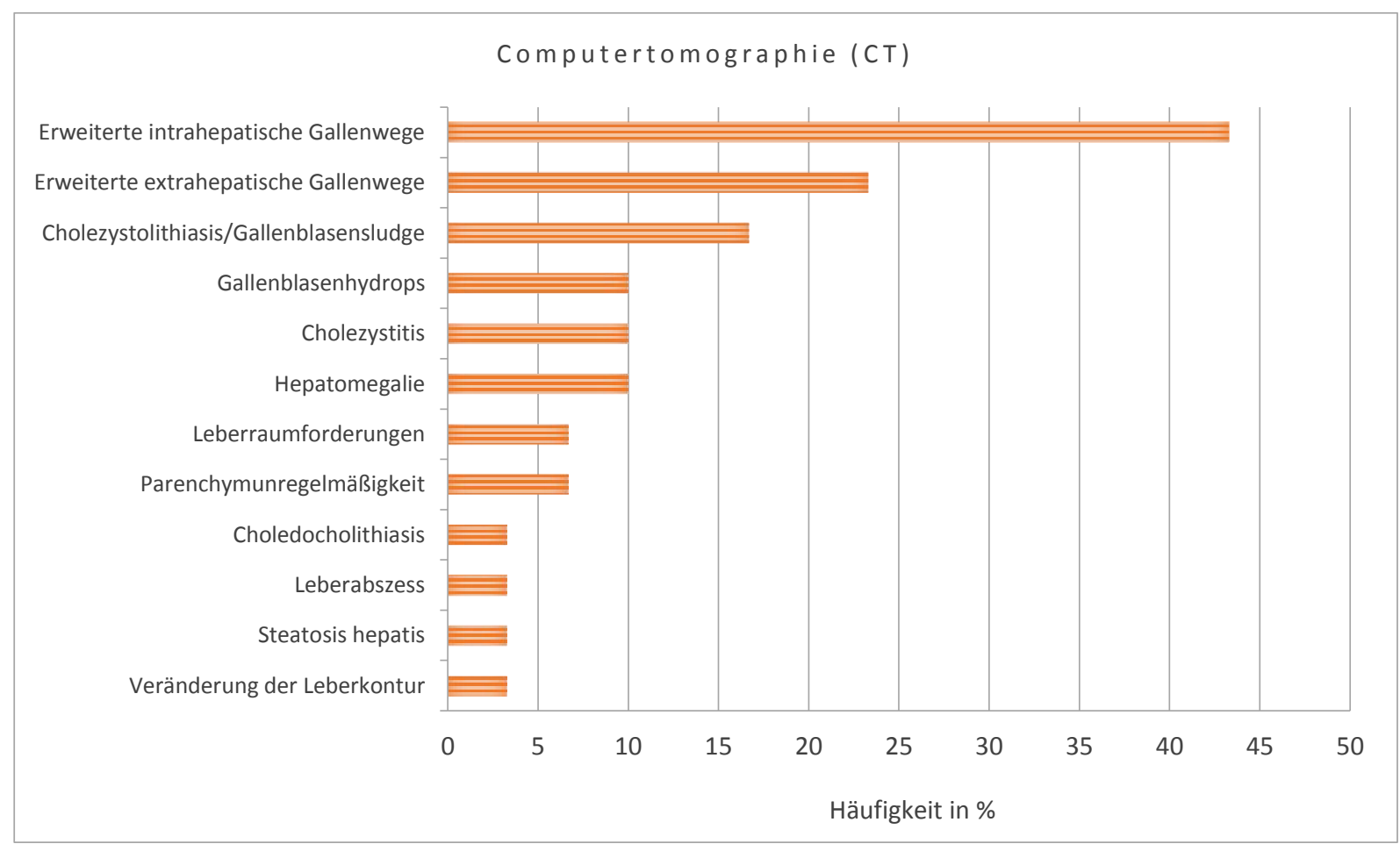

Abbildung 23: Prozentuale Verteilung pathologischer Befunde der Computertomographie (CT) bei Patienten mit ischämischer Cholangiopathie der UMG (04/200705/2015) vor Diagnosesicherung, (Kollektiv: $n=30$ von 51 Patienten)

\subsubsection{Endosonographie (EUS)}

Eine endoskopische Ultraschalluntersuchung (EUS) des oberen Gastrointestinaltraktes wurde bei 5 von 51 Patienten $(9,8 \%)$ durchgeführt. Die schriftlichen Befunde enthielten dabei eine Beurteilung der Pankreasstruktur, des Pankreasganges, der Gallenblase sowie der Gallenwege. So fanden wir bei 3 von 5 Patienten (60\%) eine extrahepatische Cholestase, bei 2 von ihnen mit einer Choledocholithiasis kombiniert. Bei einem der 5 Patienten wurde eine Cholezystolithiasis beschrieben. 


\subsubsection{Weitere bildgebende Diagnostik}

Bei je einem der 51 Patienten des Kollektivs (1,9\%) war vor Diagnosestellung eine Untersuchung mittels MRT/MRCP bzw. PET-CT durchgeführt worden. Dabei ergab die MRT/MRCP weder Auffälligkeiten der Leber, noch der Gallenwege oder der Gallenblase. In der PET-CT-Untersuchung fanden sich neben Leberabszessen keine weiteren diagnoseweisenden Befunde.

\subsection{Leberbiopsie und histopathologische Diagnostik}

Eine Leberbiospie war vor Diagnosesicherung einer ischämischen Cholangiopathie bei 6 von 51 Patienten (11,7\%) durchgeführt worden. In den vorliegenden histopathologischen Ergebnissen war bei 5 der 6 Patienten eine portale Entzündungsreaktion sichtbar. Bei zwei Patienten wurde eine kanalikuläre Cholestase beschrieben (Abbildung 24). Bei einem der 6 Patienten zeigte sich fibrinös nekrotisches Material in der Leberbiopsie.

Bei 3 Patienten von 6 Patienten (50\%) zeigten sich histopathologische Befunde, die mit einer möglicherweise medikamentös-toxischen Genese vereinbar waren. Bei 2 von 6 Patienten ergaben die Biopsieergebnisse einen Befund, der mit einer ischämischen Cholangiopathie vereinbar war. In Abbildung 24 ist weiterhin exemplarisch die Besiedlung eines via ERC geborgenen Casts mit Bakterien und Pilzen dargestellt. 

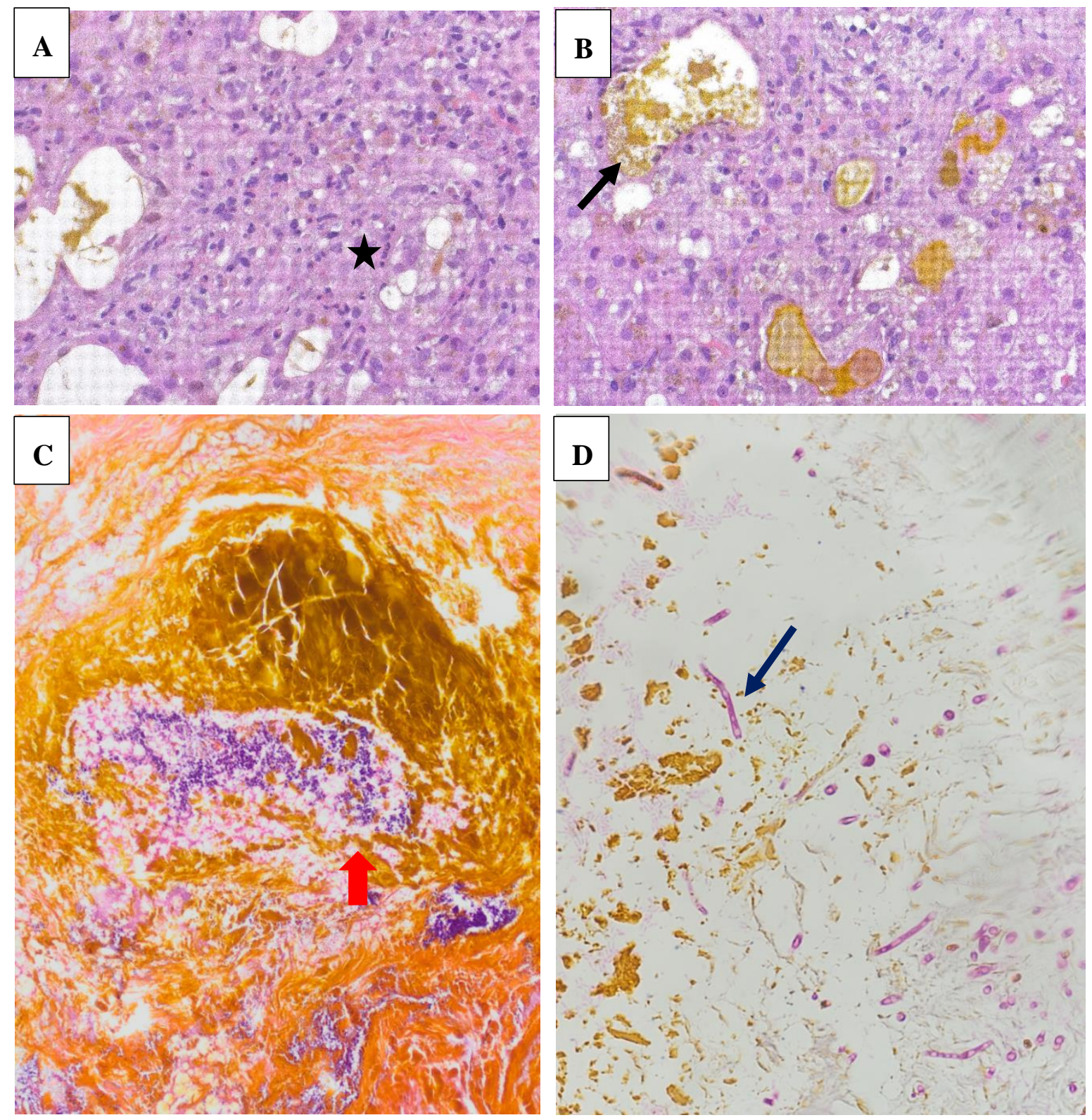

Abbildung 24: Leberbiopsie (A, B) und Casts (C, D) bei Patienten mit ischämischer Cholangiopathie: (A) Portalfeld (Stern) mit Leukozyteninfiltration bei portaler Entzündung (HE, 40x). (B) Gallenwege mit kanalikulärer Cholestase (Pfeil), (HE, 40x) (C) HE-Färbung: Casts mit Bakterienrasen (Pfeil), (HE, 40x). (D) Pilznachweis (Pfeil) in Casts (PAS, 40x)

\subsection{Bakteriologische Befunde vor Diagnosesicherung}

Vor Diagnosesicherung existierten bei 44 von 51 Patienten $(86,2 \%)$ bakteriologische Befunde. Die am häufigsten vorkommenden Erreger in der Blutkultur, Kultur des tracheobronchialen Sekrets, Katheterspitzen sowie Urinkultur vor Diagnosesicherung sind in Tabelle 3 aufgeführt. 
Bei 43 Patienten der 44 Patienten $(97,7 \%)$ lagen Ergebnisse der Blutkulturen vor. Hiervon konnten bei 21 der 43 Patienten (48,8\%) Keime in der Blutkultur nachgewiesen werden. Am häufigsten wurde Staphylococcus epidermidis bei 9 der 21 Patienten $(42,8 \%)$ nachgewiesen, davon bei 6 von 9 Patienten $(66,6 \%)$ als Oxacillin-resistenter Keim (ORSE). Als zweithäufigster Keim in der Blutkultur präsentierte sich Escherichia coli, der bei 6 von 21 Patienten (28,5\%) nachgewiesen worden war. Enterococcus faecium war bei 5 der 21 Patienten $(23,8 \%)$ diagnostiziert worden, davon bei 2 der 5 Patienten als Vancomycinresistenter Keim (VRE). Staphylococcus aureus wurde bei 4 Patienten der 21 Patienten (19\%) nachgewiesen, davon bei 2 der 4 Patienten als Oxacillin-resistenter Keim (ORSA). Bei 2 von 21 Patienten (9,5\%) war Pseudomonas aeruginosa nachgewiesen worden. Bei je 1 von 21 Patienten (4,7\%) wurde Corynebacterium jeikeium, Fusobacterium nucleatum, Massilia timoniae, Actinomyces naeslundii, Lactobacillus rhamnosus, Klebsiella pneumoniae, Enterobacter aerogenes, Serratia marcescens, Staphylococcus capitis, Staphylococcus warneri oder Staphylococcus haemolyticus in der Blutkultur nachgewiesen.

Ein Pilznachweis in der Blutkultur hatte bei 1 von 21 Patienten $(4,7 \%)$ einen Befall mit Candida albicans ergeben und bei einem weiteren Patienten (4,7\%) eine Besiedlung mit Candida krusei.

Eine mikrobiologische Beurteilung der Katheterspitzen von zentralvenösen Zugängen war bei 28 von 44 Patienten $(63,6 \%)$ erfolgt. Bei 9 von 28 Patienten $(32,1 \%)$ konnte dabei ein Keim nachgewiesen werden. Dabei präsentierte sich Staphylococcus epidermidis als häufigster Keim. Er war bei 6 von 9 Patienten $(66,6 \%)$ diagnostiziert worden, bei vier Patienten davon als ORSE-Keim. Bei 2 von 9 Patienten (22,2\%) war Escherichia coli nachgewiesen worden. Bei 2 von 9 Patienten fand sich eine Besiedlung mit Citrobacter koseri. 1 von 9 Patienten hatte einen Befall mit Enterococcus faecium und ein weiterer mit Enterobacter aerogenes.

Ein Pilznachweis an Katheterspitzen ergab sich bei 2 von 9 Patienten, davon in einem Fall mit Candida glabrata und in einem weiteren Fall mit Candida albicans.

Eine Urinkultur war bei 42 von 44 Patienten $(95,4 \%)$ durchgeführt worden. Bei 28 der 42 Patienten (66,6\%) war dabei ein Keimnachweis erfolgt. Bei 11 von 28 Patienten $(39,2 \%)$ zeigt sich als häufigster Keim im Urin Enterococcus faecalis. Escherichia coli war bei 9 von 28 Patienten $(32,1 \%)$ diagnostiziert worden, davon in 1 Fall als 3-Fach multiresistenter gramnegativer Keim (3-MRGN-Keim). Bei 6 von 28 Patienten $(21,4 \%)$ hatte man Pseudomonas aeruginosa in der Urinkultur gefunden, bei weiteren 4 von 28 Patienten $(14,2 \%)$ 
Klebsiella pneumoniae. 2 von 28 Patienten hatten einen Nachweis mit Enterobacter cloacae und weitere 2 von 28 Patienten hatten einen Befall mit Proteus mirabilis in der Urinkultur. Staphylococcus epidermidis, Citrobacter koseri, Acinetobacter baumannii, Alcaligenes faecalis oder Morganella morganii waren bei je 1 von 28 Patienten (3,5\%) im Urin nachweisbar.

Unter den Pilzen in der Urinkultur war Candida albicans am häufigsten vorzufinden bei 7 von 28 Patienten (25\%). Als zweithäufigster Pilz in der Urinkultur präsentierte sich Candida glabrata mit einem Nachweis bei 2 von 28 Patienten (7,1\%), gefolgt von Candida krusei bei einem von 28 Patienten.

Die initialen Untersuchungen des tracheobronchialen Sekrets auf Keime ergaben bei 42 von 44 Patienten $(95,4 \%)$ einen positiven Keimnachweis. Am häufigsten konnte hierbei das Darmbakterium Escherichia coli nachgewiesen werden (9 von 42 Patienten, 30,9\%). Als zweithäufigster Keim zeigte sich das Bakterium Staphylococcus aureus (12 von 42 Patienten $(28,5 \%))$, davon bei zwei Patienten als ORSA-Keim. Pseudomonas aeruginosa wurde bei 6 von 42 Patienten $(14,2 \%)$ vorgefunden. Klebsiella pneumoniae war bei 4 von 42 Patienten $(9,5 \%)$ nachgewiesen worden. 3 von 42 Patienten $(7,1 \%)$ hatten Enterobacter aerogenes. Der Nachweis von Citrobacter koseri erfolgte bei 3 von 42 Patienten. Enterobacter cloacae wurde ebenfalls bei 3 von 42 Patienten nachgewiesen. Zwei von 42 Patienten $(4,7 \%)$ hatten im Tracheobronchialesekret Proteus mirabilis und bei 2 von 42 weiteren Patienten wurde Serratia marcescens nachgewiesen. Einen Nachweis von Streptococcus agalactiae, Streptococcus costellatus, Citrobacter freundii oder Morganella morganii hatte je 1 von 42 Patienten $(2,3 \%)$. Hinsichtlich einer Pilzbesiedlung des Respirationstraktes fanden wir bei 11 von 42 Patienten $(26,1 \%)$ einen Nachweis von Hefen. Bei 8 von 11 Patienten $(72,7 \%)$ war Candida albicans diagnostiziert worden, bei 2 von 11 Patienten $(18,1 \%)$ Candida glabrata und bei 1 von 11 Patieten war Candida dubliniensis (9\%) nachgewiesen worden.

Serologische Untersuchungen, die bei 32 von 44 Patienten (72,7\%) mit ischämischer Cholangiopathie durchgeführt wurden, zeigten folgende Befunde.

Die Bestimmung der Hepatitis Serologie ergab bei 15 von 32 Patienten (46,8\%) einen negativen Befund bezüglich einer aktiven Hepatitis-A-Infektion. Eine serologische HepatitisB-Diagnostik war bei 27 von 32 Patienten (84,3\%) durchgeführt worden. Auch hier hatte sich bezüglich einer aktiven oder chronischen Infektion mit dem Hepatitis-B-Virus (HBV) durchgehend ein negativer Befund gezeigt. Bei 26 von 32 Patienten (81,2\%), mit einer 
serologischen Untersuchung auf eine bestehende Hepatitis-C-Infektion ergab die Datenanalyse, dass 25 Patienten eine negative HCV Serologie aufwiesen. Bei nur einem Patienten war die HCV-RNA positiv.

Serologische Untersuchungen hinsichtlich Cytomegalievirus (CMV) sowie Epstein-BarrVirus (EBV) waren bei 5 von 32 Patienten $(15,6 \%)$ unauffällig. Varizella-Zoster-Virus (VZV)-Serologie war ebenfalls bei 4 von 32 Patienten negativ.

Bei 21 von 44 Patienten (47,7\%) war eine Stuhldiagnostik auf pathogene Erreger durchgeführt worden. Dabei hatte sich bei 10 von 21 Patienten $(47,6 \%)$ ein positiver Befund ergeben. Hiervon war bei 7 von 10 Patienten Clostridium difficile Toxin positiv. Bei 1 von 10 Patienten war Staphylococcus aureus nachweisbar. Hefebefall im Stuhl wurde bei 3 von 10 Patienten nachgewiesen. Bei einem der drei Patienten wurde Candida glabrata identifiziert. 
Tabelle 3: Erregerspektrum der häufigsten Erreger in der

Blutkultur/tracheobronchial/Katheterspitzen/Urinkultur bei Patienten mit ischämischer Cholangiopathie der UMG (04/2007-05/2015) vor

Diagnosesicherung, (Kollektiv: $n=44$ von 51 Patienten)

\begin{tabular}{|c|c|c|c|c|}
\hline $\begin{array}{l}\text { Erreger } \\
\text { Keimnachweis }\end{array}$ & $\begin{array}{l}\text { Blutkultur } \\
21 \text { Patienten }\end{array}$ & $\begin{array}{c}\text { Tracheobronchial } \\
\text { Kultur } \\
42 \text { Patienten }\end{array}$ & $\begin{array}{c}\text { Katheterspitzen } \\
9 \text { Patienten }\end{array}$ & $\begin{array}{l}\text { Urinkultur } \\
28 \text { Patienten }\end{array}$ \\
\hline \multicolumn{5}{|l|}{ Grampositive Kokken } \\
\hline S. aureus & 4/21 (19\%) & $12 / 42(28,5 \%)$ & - & - \\
\hline $\begin{array}{l}\text { Oxacillin-resistenter } \\
\text { S. aureus }\end{array}$ & $2 / 4(50 \%)$ & $2 / 12(16,6 \%)$ & - & - \\
\hline S. epidermidis & $9 / 21(42,8 \%)$ & - & $6 / 9(66,6 \%)$ & $1 / 28(3,5 \%)$ \\
\hline $\begin{array}{l}\text { Oxacillin-resistenter } \\
\text { S. epi }\end{array}$ & $6 / 9(66,6 \%)$ & - & $4 / 6(66,6 \%)$ & - \\
\hline E. faecalis & & - & & $11 / 28(39,2 \%)$ \\
\hline E. faecium & $5 / 21(23,8 \%)$ & - & $1 / 9(11,1 \%)$ & - \\
\hline $\begin{array}{l}\text { Vancomycin-resistenter } \mathrm{E} . \\
\text { faecium }\end{array}$ & $2 / 5(40 \%)$ & - & - & - \\
\hline \multicolumn{5}{|l|}{$\begin{array}{l}\text { Gramnegative fakultativ } \\
\text { anaerobe Stäbchen }\end{array}$} \\
\hline E. coli & $6 / 21(28,5 \%)$ & $13 / 42(30,9 \%)$ & $2 / 9(22,2 \%)$ & $9 / 28(32,1 \%)$ \\
\hline 3-MRGN E. coli & - & - & - & $1 / 9(11,1 \%)$ \\
\hline Citrobacter koseri & - & $3 / 42(7,1 \%)$ & $2 / 9(22,2 \%)$ & $1 / 28$ \\
\hline Klebsiella pneumonie & - & $4 / 42(9,5 \%)$ & - & $4 / 28(14,2 \%)$ \\
\hline Enterobacter aerogenes & $1 / 21(4,7 \%)$ & $3 / 42(7,1 \%)$ & $1 / 9(11,1 \%)$ & - \\
\hline Enterobacter cloacae & - & $3 / 42(7,1 \%)$ & - & $2 / 28(7,1 \%)$ \\
\hline Serratia marcescens & $1 / 21(4,7 \%)$ & $2 / 42(4,7 \%)$ & - & - \\
\hline Proteus mirabilis & - & $2 / 42(4,7 \%)$ & - & $2 / 28(7,1 \%)$ \\
\hline \multicolumn{5}{|l|}{$\begin{array}{l}\text { Gramnegative aerobe } \\
\text { Stäbchen }\end{array}$} \\
\hline Pseudomonas aeruginosa & $2 / 21(9,5 \%)$ & $6 / 42(14,2 \%)$ & - & $6 / 28(21,4 \%)$ \\
\hline \multicolumn{5}{|l|}{ Candida } \\
\hline Candida albicans & $1 / 21(4,7 \%)$ & $8 / 42(19 \%)$ & - & $7 / 28(25 \%)$ \\
\hline Candida krusei & $1 / 21(4,7 \%)$ & & $1 / 9(11,1 \%)$ & $2 / 28(7,1 \%)$ \\
\hline Candida glabrata & - & $2 / 42(4,7 \%)$ & $1 / 9(11,1 \%)$ & $1 / 28(3,5 \%)$ \\
\hline
\end{tabular}




\subsubsection{Bakteriologische und mykologische Befunde im Galleaspirat}

Bei 15 von insgesamt 51 Patienten $(29,4 \%)$ war im Rahmen der ERC-Untersuchung aus den Gallenwegen eine Aspiration von Gallesekret zur mikrobiologischen Diagnostik erfolgt (Tabelle 4). Als häufigster Keim in der Galleflüssigkeit imponierte dabei Enterococcus faecium mit einem Nachweis bei 7 der 15 Patienten (46,6\%), hierbei war er bei 1 von 7 Patienten (14,2\%) als Vancomycin-resistenter (VRE)-Keim nachgewiesen worden. Staphylococcus epidermidis war bei 3 von 15 Patienten (20\%) nachweisbar. Bei 2 von 15 Patienten (13,3\%) fanden wir den Nachweis von Pseudomonas aeruginosa und Streptococcus viridans. Bei je 1 von 15 Patienten $(6,6 \%)$ waren Stenotrophomonas maltophila, Klebsiella pneumonie (als 3-MRGN Keim), Citrobacter freundii (3-MRGN), E. coli, Lactobacillus rhamosus und Citrobacter koseri vorzufinden.

Candida albicans wurde bei 4 Patienten von 15 Patienten (26,6\%) im Galleaspirat identifiziert. Bei 2 von 15 Patienten (13,3\%) wurde Candida dubliniensis nachgewiesen.

6 der 15 Patienten (40\%) wiesen einen Befall der Galleflüssigkeit sowohl mit Bakterien als auch mit Pilzen auf.

Tabelle 4: Erregerspektrum der häufigsten Erreger in der Galleaspirat-Kultur bei Patienten mit ischämischer Cholangiopathie der UMG (04/2007-05/2015) vor Diagnosesicherung, (Kollektiv: $\mathrm{n}=15$ von 51 Patienten)

\begin{tabular}{|l|c|}
\hline $\begin{array}{l}\text { Erreger } \\
\text { Keimnachweis }\end{array}$ & $\begin{array}{c}\text { Galleaspirat Kultur } \\
\mathbf{1 5} \text { Patienten }\end{array}$ \\
\hline Grampositive Kokken & \\
\hline E. faecium & $7 / 15(46,6 \%)$ \\
\hline Vancomycin-resistenter E. faecium & $1 / 7(14,2 \%)$ \\
\hline S. epidermidis & $3 / 15(20 \%)$ \\
\hline Gramnegative aerobe Stäbchen & \\
\hline Pseudomonas aeruginosa & $2 / 15(13,3 \%)$ \\
\hline Candida & \\
\hline Candida albicans & $4 / 15(26,6 \%)$ \\
\hline Candida dubliensis & $2 / 15(13,3 \%)$ \\
\hline
\end{tabular}




\subsection{Diagnosesicherung mittels ERC}

Neben laborchemischen, bakteriologischen, bildgebenden und ggf. bioptischen Maßnahmen wurde zunächst bei allen in der UMG behandelten Patienten mit Verdacht auf ischämische Cholangiopathie/ggf. sekundär sklerosierende Cholangitis versucht, medikamentöse Einflüsse, die eine cholestatische Hepatopathie bedingen können, auszuschließen. In der Regel wurden deshalb alle nicht lebensnotwendigen Pharmaka für 3-7 Tage abgesetzt unter ständiger Kontrolle der Laborparameter sowie auch des klinischen Zustandes. Falls diese Maßnahmen erfolglos verliefen, erfolgte die invasive ERC zum Ausschluss einer ischämischen Cholangiopathie.

Die Sicherung der Diagnose „ischämische Cholangiopathie“ erfolgte mittels endoskopischer retrograder Cholangiographie (ERC). Hierbei wurde als Diagnosezeitpunkt das Datum gewählt, bei dem in einem schriftlichen ERC-Befund die Diagnose ischämische Cholangiopathie erwähnt wurde.

Bei 46 Patienten von 51 Patienten (90,2\%) erfolgte die Diagnosestellung mittels ERC. Davon hatten 42 der Untersuchungen in der Universitätsmedizin Göttingen (UMG) stattgefunden, während die Diagnosen in den 4 übrigen Fällen in auswärtigen Krankenhäusern mittels ERC gestellt worden und in der UMG mittels erneuter Untersuchung bestätigt worden waren.

Bei 5 von 51 Patienten des Gesamtkollektivs $(9,8 \%)$ war die Diagnose ebenfalls auswärtig gestellt worden. Allerdings ließ sich anhand der zur Verfügung stehenden Unterlagen nicht sicher eruieren, auf welchem Wege und mit Hilfe welcher laborchemischer und diagnostisch bildgebender/interventioneller Verfahren die Diagnose erfolgte. Auch in diesen Fällen wurde die Diagnose im Verlauf mittels ERC in der UMG bestätigt.

Dabei konnte bei 42 der insgesamt 51 Patienten (82,3\%) Cast-Material in der ERCUntersuchung nachgewiesen werden. Nur bei einigen Patienten wurde das Castmaterial endoskopisch geborgen. Häufig wurden Gallenwegsrarefizierungen, Gallenwegsdilatationen und -strikturen beschrieben (Abbildung 25). Bei 9 von 51 Patienten $(17,7 \%)$ wurden nur Gallenwegsrarefizierungen und/oder Gallenwegsstrikturen beschrieben. 

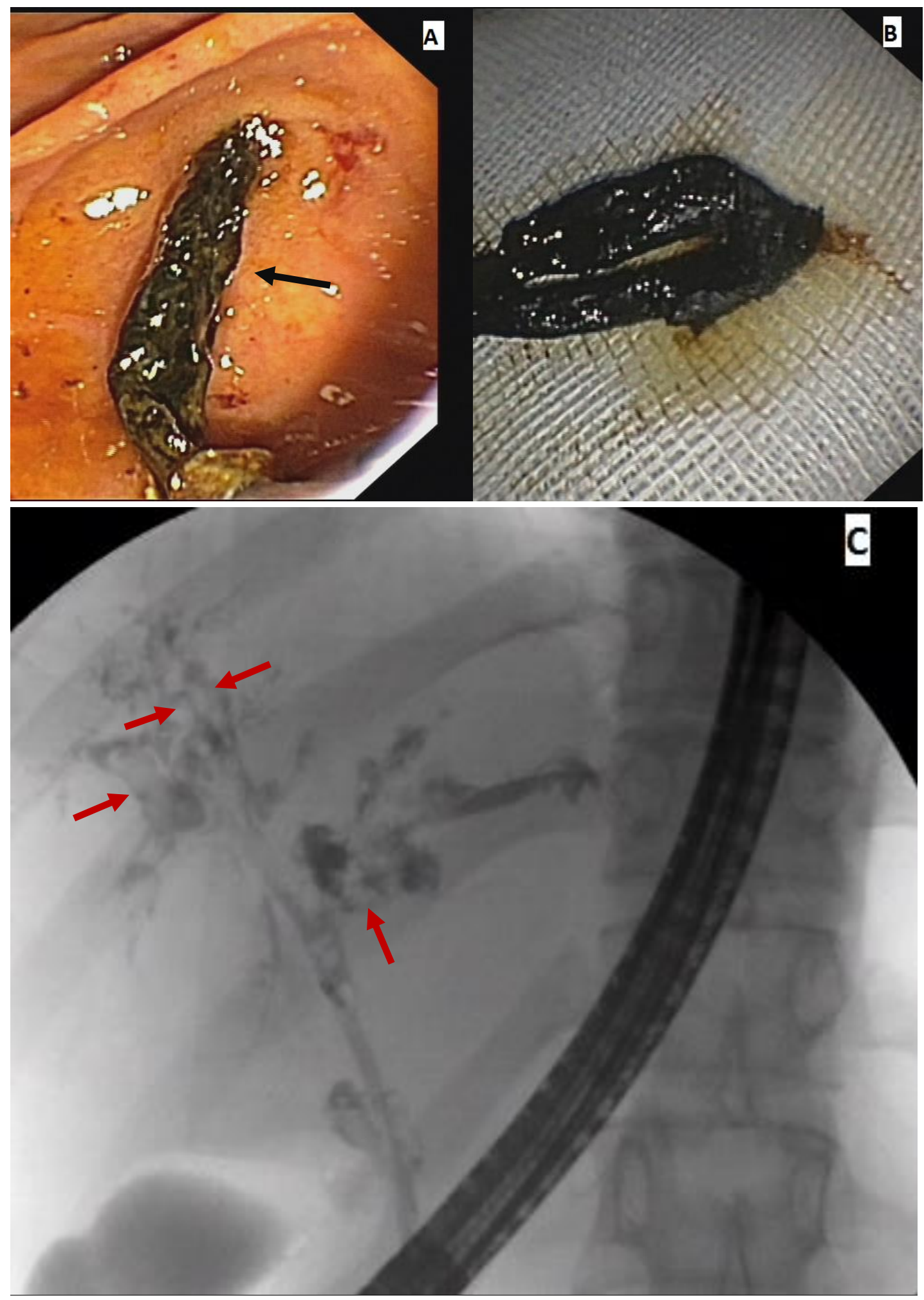

Abbildung 25: ERC-Befunde bei Patienten mit ischämischer Cholangiopathie:

(A) Entfernung von typischen Castformationen (Pfeil) nach endoskopischer Sphinkterotomie der Papilla vateri. (B) Geborgener Cast. (C) ERC zeigt Kontrastmittel-Füllungsdefekte (Pfeile) entsprechend Casts sowie Aussackungen und Rarefizierung der intrahepatischen Gallenwege. 
Die Auswertung von 42 von 51 Patienten (82,3\%), die in der UMG primär diagnostiziert wurden, ergab, daß der mediane Diagnosezeitpunkt des Krankheitsbildes ischämische Cholangiopathie bei 2,5 Monaten nach Eintritt des Ereignisses lag.

Der früheste Diagnosezeitpunkt lag innerhalb des ersten Monats, der späteste Zeitpunkt bei 42 Monaten nach dem Initialereignis. Der Mittelwert lag bei 5.4 \pm 7.7 Monaten.

Beim Großteil der Patienten (33 von 51 Patienten, 64,7\%) erfolgte die Diagnosestellung der „,ischämischen Cholangiopathie“ bereits in der ersten ERC-Untersuchung in der UMG. Bei 5 von 51 Patienten $(9,8 \%)$ war die Diagnose in der zweiten Untersuchung in der UMG gestellt worden, bei 3 der 51 Patienten (5,9\%) im Rahmen der dritten ERC in der UMG. Bei 1 von 51 Patienten $(1,9 \%)$ hatte man bis zur Diagnosefindung 4 Untersuchungen benötigt.

Bei 4 von 51 Patienten (7,8\%), die auswärts mittels ERCP diagnostiziert wurden, ist die genaue Anzahl der Untersuchungen bis zur Diagnose unklar.

Bei 5 von insgesamt 51 Patienten $(9,8 \%)$ lagen keine genauen Angaben zu den diagnostischen Verfahren vor, die zur Diagnose der Erkrankung geführt hatten (Abbildung 26).

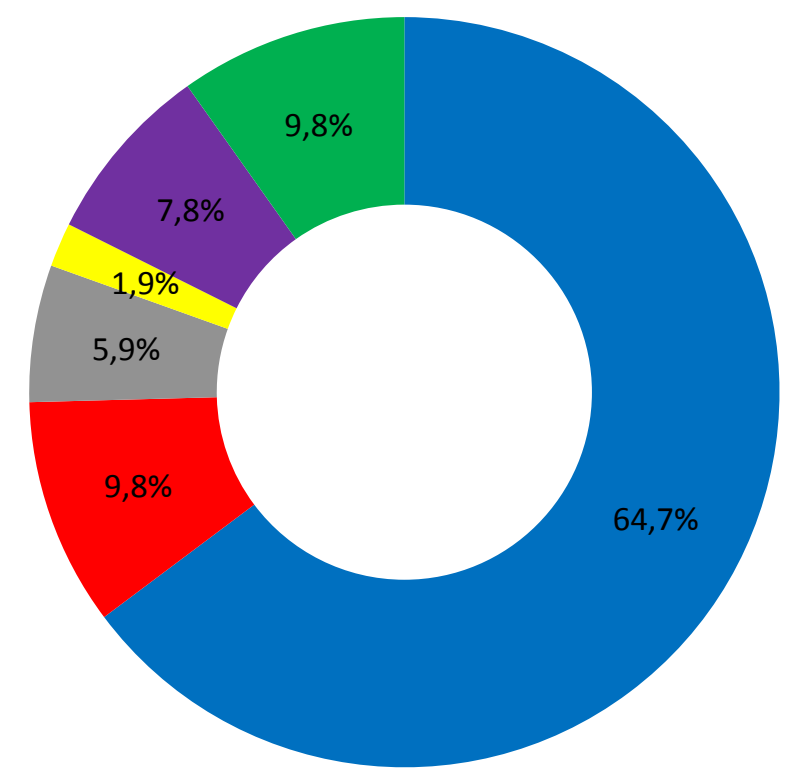

1. ERCP UMG

2. ERCP UMG

3. ERCP UMG

4. ERCP UMG

ERCP auswärts

unklar/anders diagnostiziert

Abbildung 26: Anzahl der durchgeführten ERC-Untersuchungen und prozentuale Verteilung der Untersuchungen bei Patienten mit ischämischer Cholangiopathie der UMG (04/2007-05/2015) bis zur Erstdiagnose ( $n=51$ Patienten) 


\section{Diskussion}

\subsection{Hintergrund}

Der Begriff der ischämischen Cholangiopathie beschreibt den Zustand einer lokal begrenzten oder auch ausgedehnten Gallenwegsschädigung, verursacht durch eine mangelnde Blutversorgung der Gallenwege (Deltenre und Valla 2006). Ischämisch bedingte Gallenwegsschädigungen können durch perioperative Verletzungen der hepatischen Gefäße, Thrombosen der A. hepatica, Blutgruppenunverträglichkeiten oder Abstoßungsreaktionen wie sie nach Lebertransplantationen beobachtet wurden, auftreten (Batts 1998). Auch Vaskulitiden sowie toxische Einflüsse durch intraarterielle Chemotherapie-Applikation (Floxuridine) können biliäre Ischämien verursachen (Hohn et al. 1985). Mit der Bezeichnung ischämische Cholangiopathie wurden auch solche Veränderungen bei Patienten nach Lebertransplantationen beschrieben, die trotz funktionierender biliärer Anastomose und suffizienter hepatischer Blutversorgung diese Schädigung der Gallenwege aufwiesen (Li et al. 1992; Sanchez-Urdazpal et al. 1993; Mourad et al. 2014). Als Synonym für dieses Krankheitsbild wurde auch der Begriff der Ischemic-type biliary lesions (ITBL) benutzt (Sanchez-Urdazpal et al. 1993; Heidenhain et al. 2010).

Bereits in den 70-er Jahren des vergangenen Jahrhunderts wurde berichtet, dass manche Patienten nach erfolgreicher Lebertransplantation im Verlauf kalkartige Ablagerungen in den Gallenwegen entwickelten. Diese Abgüsse (Casts) in den Gallenwegen mit konsekutiver Obstruktion führten zu tödlichen Komplikationen mit Cholangitis und Sepsis (Martineau et al. 1972; Waldram et al. 1975). Diese Veränderungen der Gallenwege werden auch als biliary cast syndrome bezeichnet. Die Pathogenese dieser Veränderungen und dieser Krankheitsentität ist weiterhin unklar (Waldram et al. 1975; Barton et al. 1995; Shah et al. 2003).

Chronisch bestehende Obstruktionen der Gallenwege wie sie beim biliary cast syndrome/ischämischer Cholangiopathie vorkommen, können nachfolgend zu einer sekundär sklerosierenden Cholangitis führen. Dabei kommt es $\mathrm{zu}$ Abflussstörungen in den Gallengängen, progredienter Cholestase, Entzündungsreaktionen der Gallenwege, Fibrose der Gallenwege mit diffusen intrahepatischen Strikturen, Gallenwegsnekrosen und auch der Ausbildung von Casts in den Gallenwegen (Keymling et al. 2013; Albert et al. 2015).

Bei der SSC lassen sich häufig Ursachen eruieren, die zu dem Krankheitsbild führen. Dazu gehören: chronische Obstruktion der Gallenwege (u.a. Choledocholithiasis), parasitäre 
Infektionen (AIDS-Cholangiopathie mit Cryptosporidium parvum-Infektion, CMVInfektion), immunologische Ursachen (Autoimmunpankreatitis mit $\operatorname{IgG}_{4}$-Cholangitis) (Ruemmele et al. 2009).

In den letzten Jahren wurde in kleineren Fallzahlen bei nicht lebertransplantierten Patienten, die jedoch einen langdauernden und komplikationsträchtigen Intensivaufenthalt aufwiesen, ein Krankheitsbild mit Cholestase, ggf. mit Ikterus und Hepatopathie beschrieben (Scheppach et al. 2001; Engler et al. 2003; Jaeger et al. 2006; Lin et al. 2014). Die bei diesem Krankenkollektiv diagnostizierten Veränderungen der Gallenwege waren mit dem Bild einer ischämischen Cholangiopathie, ggf. in Verbindung mit einer sekundär sklerosierenden Cholangitis, vereinbar.

In Anbetracht der Erkenntnis, dass bei intensivmedizinisch behandelten Patienten (häufig mit einem komplizierten Verlauf) Gallenwegsveränderungen mit entsprechenden laborchemischen und klinischen Veränderungen, die dem Bild einer ischämischen Cholangiopathie (Cholangitis), ggf. assoziiert mit einer sekundär sklerosierenden Cholangitis, auftreten können, wurde der Terminus secondary sclerosing cholangitis in critically ill patients (SC-CIP) generiert (Gelbmann et al. 2007; Voigtländer et al. 2012; Kirchner und Rümmele 2015; Ben-Ari et al. 2015).

\subsection{Epidemiologie}

Das dieser Arbeit zugrunde liegende Patientenkollektiv bestand aus 51 Patienten mit der gesicherten Diagnose der ischämischen Cholangiopathie, ggf. verbunden mit dem Bild einer sekundär sklerosierenden Cholangitis.

Patienten mit einer ischämischen Cholangiopathie auf dem Boden einer stattgehabten Lebertransplantation wurden nicht in unserem Kollektiv erfasst. 31 Patienten $(60,8 \%)$ waren männlich und 20 Patienten (39,2\%) weiblich. Das Alter unseres Patientenkollektives lag bei 59,6 \pm 14,2 Jahren (Range: 19-83 Jahre).

Wir fanden bezüglich Alters- und Geschlechtsverteilung im vorliegenden Patientenkollektiv eine Häufung beim männlichen Geschlecht mit einer Streuung über alle Altersgruppen. Diese Befunde entsprechen hinsichtlich Alters- und Geschlechtsverteilung weitgehend mitgeteilten Daten der Literatur. So beschrieben Benninger et al. (2005) ein Patientenkollektiv von fünf Patienten mit SSC nach schwerem Trauma. 80\% der Patienten waren männlich und 18-71 Jahre alt. Esposito et al. (2008) beschrieben 10 Patienten mit SCC nach Intensivaufenthalt (9 männlich, 1 weiblich) mit einem medianen Alter bei Diagnose von 52,2 Jahre und einer 
Altersverteilung von 16-68 Jahren. Bei Jaeger et al. (2006) waren es ebenfalls 10 Patienten (5 weiblich und 5 männlich) mit SSC nach Intensivaufenthalt im Alter von 41-78 Jahren zum Zeitpunkt der Diagnose. Auch Gelbmann et al. (2007) beschrieben ein Kollektiv von 26 Patienten mit SSC nach Intensivaufenthalt. 77\% der Patienten waren männlich und die Altersverteilung lag zwischen 19 und 79 Jahren.

Aufgrund dieser Daten dominiert das männliche Geschlecht bei den betroffenen diagnostizierten Patienten. Die Frage, weshalb die Krankheit vorzugsweise bei Männern auftritt, bleibt nach wie vor ungeklärt (Kirchner und Rümmele 2015).

\subsection{Patientencharakteristika und Risikofaktoren}

Bei allen Patienten unseres Kollektivs hatten schwerwiegende Krankheitsereignisse zu einem Intensivaufenthalt geführt. Somit sind diese Patienten der Gruppe der sekundär sklerosierenden Cholangitis bei Intensivaufenthalt zuzuordnen (SC-CIP). 42 von 51 Patienten (82,3\% des Gesamtkollektivs) hatten sich auf Intensivstationen der UMG befunden.

Unter den Ursachen, die bei den Patienten unserer Kollektivs zum Intensivaufenthalt geführt hatten, fanden wir an erster Stelle intrakranielle Blutungen bei 24 von 51 Patienten (47\%). Als zweithäufigste Ursache zeigten sich pulmonale Ereignisse wie respiratorische Dekompensation bei Pneumonie, acute respiratory distress syndrom (ARDS) und Lungenarterienembolie bei 9 von 51 Patienten (17,6\% des Gesamtkollektivs). Die Genese des Ereignisses scheint jedoch keine wesentliche Rolle in der Entwicklung der ischämischen Cholangiopathie, ggf. mit sekundär sklerosierender Cholangitis zu spielen. Im Patientenkollektiv, das Voigtländer et al. (2012) beschrieben, waren kardiovaskuläre und thorakale operative Interventionen (39\% der Patienten) sowie SIRS/Sepsis $(24 \%$ der Patienten) die häufigsten Gründe des Intensivaufenthaltes. Bei Gelbmann et al. (2007) waren es Patienten mit respiratorischer Dekompensation, bei Benninger et al. (2005) Patienten mit schweren Verletzungen nach Unfällen, die den Intensivaufenthalt notwendig machten. Auch Fälle nach schweren Verbrennungen wurden als eine der Ursachen angegeben (Ben-Ari et al. 2015). Bei Jaeger et al. (2006) war in der Mehrheit (50\% der Patienten) ein Trauma die Ursache des Intensivaufenthaltes, gefolgt von intrazerebralen Blutungen bei $30 \%$ der Patienten.

An Vorerkrankungen ergaben sich für unser Kollektiv anhand der retrospektiv betrachteten Patientendaten folgende Erkrankungen: arterieller Hypertonus (57,1\%), Hyperlipoproteinämie $(33,3 \%)$, Diabetes mellitus $(28,5 \%)$, Herzinsuffizienz $(21,4 \%)$ und koronare Herzkrankheit 
(KHK) mit 19\%. Zusammengefasst dominierten somit kardiovaskuläre Erkrankungen sowie Stoffwechselkrankheiten.

An hepatologischen Vorerkrankungen fand sich in unserem Kollektiv nur bei 1 von 42 Patienten $(2,3 \%)$ eine Leberzirrhose in der Vorgeschichte. Dies stimmt mit Beobachtungen anderer Autoren überein (Engler et al. 2003; Benninger et al. 2005; Gelbmann et al. 2007; Kulaksiz et al. 2008; Ben-Ari et al. 2015; Leonhardt et al. 2015), deren Patienten ebenfalls keine hepatischen oder biliären Vorerkrankungen hatten und erst im Verlauf des Intensivaufenthaltes pathologische laborchemische Werte und Gallenwegsveränderungen entwickelten. Bezüglich anderer Vorerkrankungen stellten Weig et al. (2012) fest, dass Patienten, die eine ischämische Cholangiopathie mit sekundär sklerosierender Cholangitis während des Intensivaufenthaltes entwickelten signifikant häufiger einen höheren BodyMass-Index (BMI) aufwiesen, verglichen mit Patienten bei denen es nicht zur Entstehung einer ischämischen Cholangiopathie kam $\left(37,4 \pm 6 \mathrm{~kg} / \mathrm{m}^{2}\right.$ vs. $\left.29,6 \pm 7 \mathrm{~kg} / \mathrm{m}^{2}\right)$. In unserem Kollektiv war eine Adipositas bei 9 von 42 Patienten (21,4\%) beschrieben.

Die am häufigsten benutzen Pharmaka in der Vormedikation waren beim vorliegenden Patientenkollektiv Diuretika (50\%), gefolgt von Thrombozytenaggregationshemmern und $\beta$ Betablockern bei ca. je $40 \%$ der Patienten.

In der vorhandenen Literatur finden sich keine detaillierten Angaben über Vorerkrankungen und Vormedikation bei den beschriebenen Patienten mit ischämischer Cholangiopathie und sekundär sklerosierender Cholangitis nach Intensivaufenthalt. Ihre eventuelle Rolle in der Pathogenese dieses Krankheitsbildes bleibt somit offen.

\section{$5.4 \quad$ Intensivaufenthalt}

Die Dauer des Intensivaufenthaltes betrug in unserem Kollektiv im Median 32 Tage mit einem Aufenthaltsminimum von 13 Tagen und einen Maximum von 145 Tagen. Anhand der Unterlagen konnte ermittelt werden, dass drei Patienten vor dem Aufenthalt in der UMG kardiopulmonal reanimiert wurden. Nur in einem Fall fand sich eine kardiopulmonale Reanimation während eines Intensivaufenthaltes in der UMG dokumentiert.

Von unserem Kollektiv waren 30 von 42 Patienten $(71,4 \%)$ mit Intensivaufenthalt in der UMG bereits intubiert und beatmet auf einer Intensivstation (ICU) aufgenommen worden, während 10 von 42 Patienten $(23,8 \%)$ zu diesem Zeitpunkt nicht intubiert und beatmet gewesen waren. 
Detaillierte Daten bezüglich der Beatmung hatten allerdings nur bei 20 Patienten (39,2\% des Patientengesamtkollektivs von 51 Patienten) vorgelegen. Bei diesen lag die Dauer der invasiven Beatmung im Median bei 22 Tagen mit einem Minimum von neun Beatmungstagen und einem Maximum von 75 Tagen. Bei vier unserer Patienten ist eine extrakorporale Membranoxigenierung (ECMO) und bei zwei Patienten eine Hochfrequenzoszillations(HFO) Beatmung dokumentiert.

Alle Patienten mit ischämischer Cholangiopathie/sekundär sklerosierender Cholangitis und einer Vorgeschichte mit Intensivaufenthalt, von denen in verschiedenen retrospektiven Untersuchungen berichtet wurde, waren im Rahmen eines Intensivaufenthaltes invasiv beatmet worden. Dabei waren die Dauer des Intensivaufenthaltes sowie die Beatmungsdauer sehr unterschiedlich und das Krankheitsbild trat auch bei Patienten mit einer kürzer dauernden Beatmung oder kürzeren Intensivaufenthalten auf. Die Dauer der Beatmung hatte bei 14-60 Tagen (Kulaksiz et al. 2008), 18-39 Tagen (Jaeger et al. 2006), bzw. 13-62 Tagen (Benninger

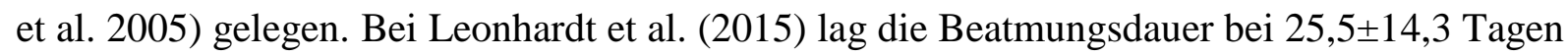

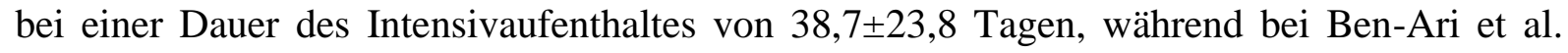
(2015) die Dauer des Intensivaufenthaltes 129,2 \pm 53 Tage betrug.

Gelbmann et al. (2007) berichteten in ihrem Kollektiv über eine Beatmungsdauer von 40,7 $\pm 32,9$ Tage (Range: 10-124 Tage). Dabei hatten alle 17 Patienten eine PEEP (positive endexipiratory pressure) Beatmung $>10 \mathrm{~cm} \mathrm{H}_{2} \mathrm{O}, 9 / 17$ Patienten hatten eine HFO Beatmung und 12/17 Patienten eine Bauchlage während der Ventilation. Die invasive Beatmung, v.a. mit erhöhtem positiven endexspiratorischen Druck (PEEP) wird als möglicher Einflussfaktor mit einem negativen Effekt auf die mikrozirkulatorische Blutversorgung erwähnt (Gelbmann et al. 2007). Weig et al. (2012) stellten fest, dass Patienten, die eine SSC entwickelten im Vergleich zu anderen Patienten während des Intensivaufenthaltes signifikant häufiger längere Bauchlagerungen während der Beatmung hatten.

Eine ischämische Cholangiopathie mit sekundär sklerosierender Cholangiopathie kann durch Schädigungen des Gallenwegsepithels, besonders bei gestörter arterieller Durchblutung der größeren A. hepatica-Äste oder der kleineren peribiliären Gefäßplexus entstehen (Abdalian und Heathcote 2006). Auch eine postoperative Thrombose der hepatischen Arterien (Batts 1998) oder eine intraarterielle Chemotherapeutika-Applikation über die hepatischen Arterien mit Floxuridine (Hohn et al. 1985) können nach bisheriger Datenlage das Krankheitsbild einer ischämische Cholangiopathie mit sklerosierender Cholangitis verursachen. Daher haben wir in unserem Patientenkollektiv die durchgeführten Maßnahmen auf der Intensivstation analysiert. Dabei waren in 32 Fällen während ihres Aufenthaltes auf einer Intensivstation 
größere operative Eingriffe (neurochirurgische - $(34 \%)$, kardiovaskuläre - $(25,5 \%)$ und abdominal-chirurgische - $(19,1 \%)$ Eingriffe) durchgeführt worden.

Kleinere Eingriffe wie Tracheotomien, Anlage von intracerebralen Drainagen, PEG-Sonden Anlagen oder Herzkatheteruntersuchungen waren in jeweils 34, 16, 7 und 3 Fällen vorgenommen worden. Alle diese Eingriffe können möglicherweise eine Minderdurchblutung triggern. Lison et al. (2011) zeigten, dass Patienten nach größeren operativen Eingriffen zu einer gesteigerten Aktivität des Gerinnungssystems neigten. Dabei waren die routinemäßig durchgeführten laborchemischen Tests (Prothrombinzeit (Quick-Wert) und partielle Thromboplastinzeit (PTT)) normal. Der Quick-Wert in unserem Kollektiv war mit einem niedrigsten Tagesmedianwert von 68,5\% während der ausgewerteten Intensivaufenthaltsphase leicht erniedrigt oder normal gemessen.

Einen weiteren Aspekt, der möglicherweise bei der Entstehung der ischämischen Cholangiopathie eine Rolle spielen könnte, stellt die medikamentöse Therapie während des Intensivaufenthaltes dar.

23 Patienten unseres Kollektivs erhielten eine medikamentöse Kreislaufunterstützung mit vasokonstriktorisch wirksamen Pharmaka. Bei allen 23 Patienten war dabei Noradrenalin (Norepinephrin) verabreicht worden. Bei jeweils zwei Patienten war eine Kombinationstherapie aus Noradrenalin und Dobutamin bzw. Vasopressin und bei drei Patienten eine Kombination aus Noradrenalin und Adrenalin (Epinephrin) vorzufinden. Diese Medikamente können den Blutfluss und somit die Blutversorgung der inneren Organe, z.B. auch der Gallenwege, beeinflussen.

So berichteten Krejci et al. (2006) aus experimentellen Untersuchungen an Schweinen, dass Adrenalin und Noradrenalin trotz Steigerung des systemischen Blutflusses den Blutfluss vom Mesenterialsystem umverteilten und die Mikrozirkulation in einigen gastrointestinalen Organen wie Jejunalmukosa und Pankreas reduzierten.

Auch Dopamin und Dobutamin zeigten in tierexperimentellen Untersuchungen (Schweine) zwar eine Steigerung der kardialen Auswurfleistung, hatten jedoch keinen signifikanten Einfluss auf die Verbesserung der Mikrozirkulation der gastrointestinalen Organe (Hiltebrand et al. 2004).

Weitere Pharmakagruppen, die in der Intensivmedizin häufig benutzt werden sind Anästhetika und Analgetika. Mehrere Fallberichte beschrieben Cholestase und Veränderungen der Gallenwege durch das Anästhetikum Ketamin, die bei Patienten mit chronischem Ketamin-Abusus vorkamen (Wong et al. 2009; Lo et al. 2011). Turkish et al. (2013) wie auch Lui et al. (2014) berichteten über Patienten nach Ketamin-Abusus, die auch 
histologisch eine periduktale Fibrose und duktale Proliferation aufwiesen. In der ERC zeigte sich hierbei ein Bild von Cholestase mit Gallenwegsstrikturen oder -dilatationen. Die Pathogenese der Ketamin-Wirkung auf die Gallenwege blieb letztendlich unklar.

Ein weiteres, in der Intensivmedizin häufig verwendetes Pharmakon ist Propofol. Propofol hatte in einer tierexperimentellen Untersuchung bei Ratten bei schockinduzierten Organschädigungen durch Hemmung der inflammatorischen Cytokine eine protektive Wirkung auf innere Organe (Leber, Lunge, Niere und Intestinum) (Lee et al. 2008).

In unserem Patientenkollektiv erhielten 28 der 42 Patienten (66,6\%) Ketamin und nur für neun Patienten $(21,4 \%)$ war die Gabe von Propofol dokumentiert.

In der publizierten Literatur über Patienten mit ischämischer Cholangiopathie bei Intensivbehandlungen sind bisher keine Mitteilungen über den Einfluss von Anästhetika/Analgetika vorhanden. Der Einfluss dieser Pharmaka ist somit offen bei diesem Patientengut.

Weiterhin untersuchten wir in unserem Kollektiv die Häufigkeit und Art der antimikrobiellen Therapie während des Intensivaufenthaltes. Bei 29 von 42 Patienten (69\%) war eine antibiotische Therapie mit Penicillin-Antibiotika erfolgt, darunter am häufigsten mit einer Kombination aus Piperacillin/Sulbactam oder Piperacillin/Combactam sowie mit Amoxicillin/Clavulansäure. Bei der am zweithäufigsten eingesetzten Substanzgruppe handelte es sich um die Carbapeneme (Meropenem und Imipenem) bei 24 von 42 Patienten $(57,1 \%)$. Cephalosporine waren die am dritthäufigsten eingesetzten Antibiotika bei 52,3\% der Patienten.

In der Literatur sind cholestatische Veränderungen mit entsprechendem laborchemischem und histologischem Korrelat (portale Entzündung, duktale Nekrose) vor allem für Amoxicillin und Clavulansäure beschrieben worden (Ryley et al. 1995; Belardo et al. 2013). Auch für Piperacillin und Imipenem/Cilastatin oder Cephalosporine sind Fälle von Cholangiopathien oder Cholestase dokumentiert (Quattropani et al. 2001; Algahtani et al. 2015; Fachinfo zu den erwähnten Antibiotika).

\subsection{Diagnostik}

\subsubsection{Laborparameter}

In den Publikationen verschiedener Autoren wird erwähnt, dass bei Intensivpatienten, die das Bild einer ischämischen Cholangiopathie mit sekundär sklerosierender Cholangitis entwickelten, keine Vorerkrankungen der Leber oder der Gallenwege bekannt waren. Zudem 
hatten die Patienten initial normwertige Cholestasewerte, bei denen sich erst im Krankheitsverlauf ein starker Anstieg entwickelte (Benninger et al. 2005; Esposito et al. 2008; Kulaksiz et al. 2008; Ben-Ari et al. 2015). Diese Beobachtungen treffen auch auf unser Patientenkollektiv zu.

Wir haben im vorliegenden Patientengut den Verlauf verschiedener Laborparameter (Leberund Cholestasewerte, Hämoglobin, Leukozyten, Kreatinin, Quick-Wert, arterielle Blutgasanalyse) vom Zeitpunkt der Aufnahme auf Intensivstation sowie für die nachfolgenden sechs Wochen während dieses Aufenthaltes analysiert. Bei den Parametern der arteriellen Blutgasanalysen betrug diese Beobachtungsphase vier Wochen. Daten über eine solche „Langzeitbeobachtung“ der erwähnten Laborparameter bei Intensivpatienten mit ischämischer Cholangiopathie wurden in der Literatur bisher nicht beschrieben.

Bei den Leberwerten zeigten sich diese bei allen 42 von 51 Patienten am Tag des Ereignisses (Tag 1) im Tagesmedian im Normbereich. Auch der Wochenmedian zeigte in der ersten Woche normale bzw. grenzwertig normale Leberwerte.

Betrachtet man die Cholestaseparameter, so dominierte bei unserem Patientenkollektiv mit ischämischer Cholangiopathie ein Anstieg der $\gamma^{-G T}$ und AP sowie des Gesamtbilirubins. In der ersten Woche lagen diese Werte im Referenzbereich. Im Verlauf wies die AP eine kontinuierliche Erhöhung auf mit einem maximalen Wochenmedianwert in der 6. Woche (Ende der Beobachtungsphase). Der Anstieg des Wochenmedianwertes betrug ca. das 16Fache des Ausgangswertes. Die y-GT war in der ersten Woche (auf Wochenmedian bezogen) im Normbereich und erreichte einen maximalen Wochenmedianwert in der 3. Woche mit einer Erhöhung auf das ca. 39-Fache des Ausgangswertes. Das Gesamtbilirubin zeigte eine Erhöhung der Werte um das ca. 12-Fache im Verlauf. Der maximale Wochenmedianwert des Gesamtbilirubins wurde, wie auch bei der AP, in der 6. Woche erreicht.

Die Transaminasen zeigten eine maximale Erhöhung der Werte bis auf das ca. 3-Fache des Ausgangswertes (AST) und auf das ca. 8-Fache des Ausgangswertes bei ALT. Dabei lagen die maximalen AST Wochenmedianwerte in der sechsten Woche, während die ALT ihren höchsten Wochenmedianwert in der dritten Woche erreichte und im Verlauf bis zur sechsten Woche rückläufig war.

Auch in der publizierten Literatur wurde ein stärkerer Anstieg der Cholestaseparameter verglichen mit dem der Transaminasen bei Patienten mit ischämischer Cholangiopathie und Intensivaufenthalt beobachtet.

Lin et al. (2014) haben mehrere Publikationen über Intensivpatienten mit ischämischer Cholangiopathie und sekundär sklerosierender Cholangitis zusammenfassend ausgewertet (88 
Patienten). Hierbei zeigte sich, dass das Gesamtbilirubin wie auch die $\gamma_{-}$GT auf das ca. 10Fache, die AP auf das ca. 6-Fache des Normbereichs erhöht waren. Die Transaminasen AST und ALT lagen nur bei 20 Patienten vor. Sie waren auf das ca. 3-Fache des Normbereichs erhöht. Der Zeitpunkt, wann im Krankheitsverlauf obige Cholestase- und Leberparameter bestimmt wurden, wird in der Analyse nicht mitgeteilt.

Ben-Ari et al. (2015) berichteten bei vier Schwerverbrannten mit ischämischer

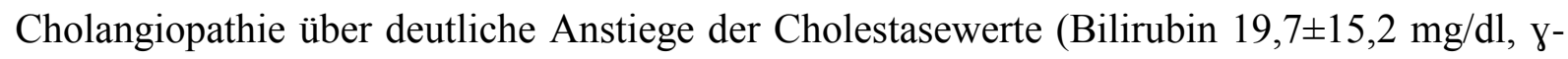
GT $4045 \pm 2056$ U/l, AP 2482 \pm 4964 U/l, jeweils Mittelwert \pm SD) ca. 50 Tage nach Aufnahme. Die AST lag bei $207 \pm 42$ U/l, die ALT bei $158 \pm 29$ U/l. Leider sind in der Publikation keine Referenzbereiche aufgeführt.

Bei Kulaksiz et al. (2008) zeigten sich die Transaminasen und Cholestasewerte vor Erkrankung im Referenzbereich. Bereits ab dem ersten Tag nach Ereignis waren jedoch steigende Werte zu beobachten. Nach einem 14-tägigen Verlauf zeigte sich ein Anstieg der Transaminasen auf das ca. 4-7-Fache verglichen mit Werten vor der Erkrankung. Der Anstieg des Gesamtbilirubins betrug ca. das 8-Fache, der der AP ca. das 3-Fache, während die y-GT einen ca. 20-Fachen Anstieg nach 14 Tagen zeigte. Auch zum Zeitpunkt der Diagnose war der Anstieg der Cholestasewerte (AP, y-GT und Gesamtbilirubin) vorherrschend.

Der Quick-Wert als Parameter der Lebersyntheseleistung imponierte bei unserem Patientenkollektiv insgesamt stabil. Dabei gilt es jedoch zu berücksichtigen, dass eventuelle, durch Vitamin K und Gerinnungsfaktoren hervorgerufene Korrekturen nicht berïcksichtigt wurden.

Thomas et al. (2014) beobachteten bei drei Patienten mit ischämischer Cholangiopathie und sekundär sklerosierender Cholangitis Quick-Werte von 55\%, 74\% und 91\%. Ben-Ari et al. (2015) wie auch Voigtländer et al. (2012) berichteten über INR-Werte von 1,96 $\pm 1,37$ (Mittelwert \pm SD) bzw. 1,13 (Median). Angaben zu Referenzwerten und dem Verlauf dieser Parameter fehlen jedoch bei diesen aufgeführten Publikationen. In der publizierten Literatur fanden sich keine weitere Angaben über Gerinnungsparameter und deren Verlauf bei Patienten mit diesem Krankheitsbild.

Auffallend beim vorliegenden Krankengut mit ischämischer Cholangiopathie war, dass Patienten nach Intensivaufnahme deutlich erniedrigte $\mathrm{Hb}$-Werte innerhalb der ersten Woche entwickelten. Diese erniedrigten Hb-Werte blieben während des Beobachtungszeitraums von sechs Wochen bestehen. 
Die Hb Werte fluktuierten zwischen 8-9 g/dl, d.h. die Intensivpatienten, die eine ischämische Cholangiopathie ggf. mit sekundär sklerosierender Cholangitis entwickelten, wiesen über einen Zeitraum von mehreren Wochen eine Anämie auf.

Voigtländer et al. (2012) berichteten, dass der Hb-Wert zum Zeitpunkt der ERC (Diagnosezeitpunkt) im Median bei 10,2 g/dl lag. Angaben über den Hb Verlauf während des Intensivaufenthaltes sind nicht mitgeteilt. Bei Benninger et al. (2005) hatten alle Patienten fünf Patienten in der frühen Phase des Intensivaufenthaltes im Minimum Hb-Werte von 7,3 $\mathrm{g} / \mathrm{dl}, 2,4 \mathrm{~g} / \mathrm{dl}, 7,2 \mathrm{~g} / \mathrm{dl}, 8,6 \mathrm{~g} / \mathrm{dl}$ und 7,9 g/dl und wiesen somit eine ausgeprägte Anämie auf. Alle diese Patienten erhielten multiple Bluttransfusionen.

In der restlichen bisher publizierten Literatur finden sich keine Angaben über den $\mathrm{Hb}$ Verlauf während der Entwicklung einer ischämischen Cholangiopathie. Ebenfalls finden sich, bis auf Voigtländer et al. (2012) keine Angaben über Hb/Anämie zum Zeitpunkt der Diagnosestellung.

Welchen Einfluss die festgestellte Anämie bei unserem Krankengut auf die Entwicklung der ischämischen Cholangiopathie aufweist, lässt sich derzeit nicht definitiv beantworten. Es ist jedoch bekannt, dass es einen Zusammenhang zwischen Anämie und Morbidität bzw. Mortalität gibt. Hare et al. (2013) kommen in ihrer Review zur Schlussfolgerung „anemia is associated with increased morbidity and mortality“. Desormais et al. (2014) hatten in ihrer Analyse von 925 Patienten mit peripherer arterieller Verschlusskrankheit (pAVK) häufigere Komplikationen hinsichtlich Amputationen und Mortalität bei der Präsenz einer Anämie (Hb $<$ 8,2 mmol/l bei Männern, entsprechend 13,2 g/dl und $\mathrm{Hb}<7,6 \mathrm{mmol} / \mathrm{l}$, entsprechend 12,2 g/dl bei Frauen) verglichen zum Kollektiv mit höheren Hb Werten (keine Anämie). Auch Go et al. (2006) haben in ihrer Studie (ANCHOR-Studie) mit großem Kollektiv (59.772 Patienten) gezeigt, dass bei Patienten mit chronischen Nierenerkrankungen und chronischer Herzinsuffizienz ein erniedrigter $\mathrm{Hb}<13 \mathrm{~g} / \mathrm{dl}$ eine höhere Mortalität und längere Hospitalisierung zur Folge hatte.

Der Kreatinin-Wert blieb im Median bei allen Patienten in unserem Kollektiv in den ersten 42 Tagen normwertig bis leicht erhöht mit einem maximalen Tagesmedian von 1,3 mg/dl. Darunter sind jedoch Patienten, die auch einer CVVH oder Dialyse unterzogen wurden, miterfasst. Weig et al. (2012) beschrieben, dass sowohl in der Patientengruppe, die während des Intensivaufenthaltes eine ischämische Cholangiopathie mit sekundär sklerosierender Cholangitis entwickelten als auch bei Patienten bei denen dies nicht der Fall war, in der ersten Woche die Anzahl der Patienten mit Nierenersatztherapie und deren Einsatz-Frequenz in 
beiden Gruppen gleich verteilt war. Erst in der vierten Woche hatten die Patienten mit ischämischer Cholangiopathie signifikant häufiger Nierenersatztherapien. Die Rolle der renalen Insuffizienz und des Kreatinin-Anstieges in der Entstehung einer ischämischen Cholangiopathie lassen sich derzeitig nicht beurteilen. So lagen die Kreatinin Medianwerte zum Zeitpunkt der Diagnose bei Voigtländer et al. (2012) bei $0,81 \mathrm{mg} / \mathrm{dl}$ und somit im Normbereich, während bei Ben-Ari et al. (2015) der Kreatinin Mittelwert 2,2 $\pm 2,18$ mg/dl betrug.

Der Leukozytenwert zeigte sich in unserem Kollektiv im Wochenmedian in den ersten vier Wochen normwertig und erst ab der 5. Woche und in der 6. Woche waren die

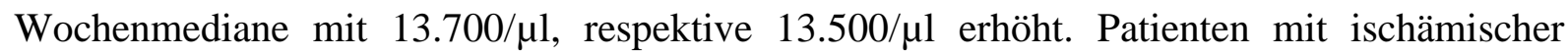
Cholangiopathie und sekundär sklerosierender Cholangitis haben ein höheres Risiko für rezidivierende Cholangitiden (Voigtländer et al. 2015). Das Krankheitsbild kann auch zu einem tödlichen septischen Schock mit Organversagen führen (Kulaksiz et al. 2008; Ben-Ari et al. 2015). Jaeger et al. (2006) stellten fest, dass nach interventioneller Therapie mittels ERC der Wert der Leukozyten um ca. 30\% fiel.

Letztendlich muss konstatiert werden, dass es in der vorliegenden Literatur nahezu keine Berichte über den Verlauf von Kreatinin, Leukozyten und Lebersyntheseparametern (QuickWert, Albumin) gibt.

Die arterielle Blutgasanalyse gilt als Goldstandard zur Beurteilung der Oxigenierung, der Ventilation und des Säure-Basen-Haushaltes bei intensivmedizinischen Patienten (Tavakol et al. 2013). Die Auswertung der Parameter der arteriellen Blutgasanalyse $\left(\mathrm{pO}_{2}, \mathrm{pCO}_{2}, \mathrm{pH}\right.$ und Laktat) als Hinweis auf Oxygenierung, Säure-Basen-Status und Gewebsischämie zeigten sich in unserem Kollektiv, gemessen vom Tag 1 bis Tag 30 des Intensivaufenthaltes als normwertig. Der arterielle $\mathrm{pO}_{2}$-Wert lag ab dem zweiten Tag durchgehend im physiologischen Bereich (am Tag 1 grenzwertiger Wert von $57 \mathrm{mmHg}$ ). Somit kann dies auf eine gute Oxygenierung der Patienten deuten. Hierbei muss man sich jedoch bewusst sein, dass diese globalen Parameter der arteriellen Blutgasanalyse nur unspezifische und insensitive Indikatoren für die Oxygenierung auf regional lokaler Gewebsebene darstellen (MacIntyre 2014)

Weig et. al. (2012) verglichen insgesamt 20 Patienten mit Intensivaufenthalt (fünf Pat. mit SSC und 15 ohne SSC). Dabei zeigte sich der Oxygenierungs-Index $\left(\mathrm{pO}_{2} / \mathrm{FiO}_{2}\right)$ in beiden Gruppen etwa gleich erniedrigt $(90.0 \pm 37.9 \mathrm{mmHg})$. 
Die Laktat-Werte waren in unserem Kollektiv mit Ausnahme des ersten Tages $(2,1 \mathrm{mmol} / \mathrm{l})$ durchgehend im Normbereich (Tagesmediane). Eine Erhöhung der Laktatwerte im Blut wird in der Literatur als Marker zur Einschätzung der Gewebsminderperfusion bei Patienten mit Schock oder Sepsis erwähnt und wird auch als Indikator einer Minderperfusion der Organe angesehen (Andersen et al. 2013). Die Konzentration von Laktat in der arteriellen Blutgasanalyse gibt nur den Wert wieder, der durch Bildung und Verbrauch im Gesamtorganismus entsteht (Thomas 2012; Soni et al. 1993). In Anbetracht der bei unserem Patientenkollektiv festgestellten Anämie sowie den nur unwesentlich veränderten Parametern der arteriellen Blutgasanalyse besteht bei unserem Patientengut die Konstellation einer anämischen Hypoxie (Ganong 2005), d.h. normaler arterieller $\mathrm{pO}_{2}-\mathrm{Wert}$ bei erniedrigtem $\mathrm{Hb}$ Wert. Dies spricht für einen verminderten $\mathrm{O}_{2}$-Transport. Auch hierbei muss darauf hingewiesen werden, dass diese gemessenen Parameter keinen direkten Rückschluss auf die regional-lokalen Verhältnisse der Gewebsoxygenierung/Gewebsperfusion in verschiedenen Organen zulassen. In der Regel wird für die Kompensation einer (akuten) Anämie die myokardiale $\mathrm{O}_{2}$-Versorgung angesehen. Es existiert gegenwärtig jedoch keine Untersuchung, die diese Annahme zweifellos beweist. Es gibt durchaus Hinweise, dass für andere Organe als das Herz die Schwelle zur Kompensation einer Anämie niedriger liegt als im Myokardgewebe (Meier et al. 2012).

Das Leberparenchym besitzt mit den hepatischen Arterien und der Vena portae eine doppelte Blutversorgung. Die Versorgung des biliären Systems, besonders jedoch der perihilären und intrahepatischen Gallenwege, erfolgt ausschließlich über Äste der A. hepatica, auch bekannt als peribiliärer vaskulärer Plexus. Daher ist das Gallengangsepithel besonders anfällig bei Veränderungen des Blutflusses der größeren A. hepatica-Äste aber auch der kleineren peribiliären Gefäße (Batts 1998; Ruemmele et al. 2009).

\subsubsection{Mikrobiologische Befunde}

Schwere Infektionen und Sepsis, besonders mit gramnegativen Keimen (z.B. Escherichia coli) können durch Herunterregulation der basolateralen oder kanalikulären Transporter der Gallensäuren zu einer hepatischen Ischämie oder Cholestase führen (Chand und Saynal 2006). In unserem Kollektiv mit ischämischer Cholangiopathie wurde das Erregerspektrum und dessen Häufigkeit in verschiedenen Kompartimenten (Blut, Trachea, Stuhl, zentralvenöse Katheter) vor Diagnosesicherung untersucht. 
Als häufigster Keim in der Blutkultur fand sich Staphylococcus epidermidis in 9 von 21 Patienten (42,8\%), hiervon bei 6 von 9 Patienten als Oxacillin -resistenter (ORSE) Keim. Escherichia coli war als zweithäufigster Keim bei 6 von 21 Patienten (28,5\%) nachweisbar. Als häufigster Keim in zentralvenösen Kathetern fand sich Staphylococcus epidermidis bei 6 von 9 Patienten (66,6\%). In der Urinkultur war Enterococcus faecalis am häufigsten nachweisbar, gefolgt von Escherichia coli. Der häufigste Keim im Tracheobronchialsystem war Escherichia coli.

Infektionen des biliären Systems spielen möglicherweise eine Rolle bei der Entstehung der ischämischen Cholangiopathie mit Ausbildung von „Casts“ z.B. durch Dekonjugation von Bilirubin in unlösliche Verbindungen oder durch direkte Keiminvasion der Schleimhaut (Gelbmann et al. 2007). Auch bei der AIDS-Cholangiopathie werden Keime wie Cryptosporidium parvum oder Cytomegalieviren in Zusammenhang mit der Entstehung der Cholangiopathie mit sekundär sklerosierender Cholangitis gebracht (Abdalian und Heathcote 2006).

In unserem Kollektiv war eine mikrobiologische Untersuchung des Gallesekrets, das endoskopisch aus dem Gallenwegssystem aspiriert wurde in 15 Fällen $(29,4 \%)$ im Rahmen der Diagnostik erfolgt. Als häufigster Erreger fand sich Enterococcus faecium bei fast der Hälfte der Patienten (7 von 15 Patienten, 46,6\%), davon einmal als Vancomycin-resistenter Keim (VRE). Candida albicans wurde bei 4 von 15 Patienten (26,6\%) nachgewiesen. $\mathrm{Zu}$ erwähnen ist, dass 6 von 15 Patienten (40\%) einen gleichzeitigen Pilz und Bakteriennachweis in der Galle aufwiesen.

Voigtländer et al. (2015) untersuchten mikrobiologisch das Galleaspirat bei 97 Patienten mit ischämischer Cholangiopathie und sekundär sklerosierender Cholangitis. Dabei konnten bei 94\% der Patienten Keime in der Gallekultur nachgewiesen werden. Die am häufigsten nachgewiesenen Keime waren Enterococcus spp. (51 von insgesamt 233 nachgewiesenen Keimen), gefolgt von Escherichia coli (12 von 233 nachgewiesenen Keimen). Candida albicans fand sich als häufigster Pilzbefall in diesem Kollektiv (24 von 233 Keimen). Fast die Hälfte der Patienten (45,6\%) wies mehrere Keime in der Gallekultur auf. In der Blutkultur hatten die Patienten häufiger negative Befunde verglichen mit der Gallekultur (8 vs. 20 Patienten). Das Keimspektrum in der Gallekultur führte bei 64\% der Patienten zu einer Anpassung der antimikrobiellen Therapie (Voigtländer et al. 2015). 


\subsubsection{Bildgebende Diagnostik}

In unserem Kollektiv mit ischämischer Cholangiopathie wurden die verschiedenen bildgebenden Verfahren, die bis zur endgültigen Diagnosesicherung vorgenommen worden waren, analysiert. Die transabdominale Sonographie war eine der leicht zugänglichen und durchführbaren Untersuchungsmethoden. Sie besitzt eine hohe Spezifität zur Identifizierung von Gallenwegsobstruktionen (Siddique und Kowdley 2012).

In unserem Patientenkollektiv war die Mehrheit der Patienten (43 von 51, 84,3\%) einer transabdominalen Sonographie unterzogen worden. Die Indikation bestand in den meisten Fällen in der Abklärung von erhöhten Cholestasewerten.

Bezüglich der Leber entdeckten wir als häufigste pathologische Sonographiebefunde eine Hepatomegalie bei 19 von 43 Patienten $(44,1 \%)$ und eine Steatosis hepatis in 7 Fällen (16,2\%). Ein zirrhotischer Umbau des Lebergewebes wurde bei 2 von 43 Fällen $(4,6 \%)$ beschrieben. Pathologische Befunde der Lebergefäße wurden in keinem Fall erwähnt.

Die Befunde der Gallenwege ergaben bei 14 von 43 Patienten (32,5\%) eine extrahepatische Gallenwegserweiterung und bei 13 von 43 Patienten (30,2\%) eine Erweiterung der intrahepatischen Gallenwege. Bei keinem der Patienten wurde die Diagnose der ischämischen Cholangiopathie/sekundär sklerosierenden Cholangitis mittels Abdomensonographie gestellt. Auch im Patientenkollektiv von Gelbmann et al. (2007) ergab die Abdomensonographie mit Duplexsonographie regelrechte Befunde der arteriellen und venösen Lebergefäße bei allen Patienten mit ischämischer Cholangiopathie und sekundär sklerosierender Cholangitis. Voigtländer et al. (2012) berichteten, dass bei 13 von 44 Patienten die Befunde der Abdomensonographie das Vorliegen einer sekundär sklerosierenden Cholangitis „suggerierten“. Die dabei erhobenen sonographischen Befunde sind jedoch in der Publikation nicht aufgeführt.

Benninger et al. (2005) berichteten, dass in ihrem Kollektiv von fünf Patienten in den ersten 1-2 Monaten bei vier Patienten die Leber normal groß war, während nur ein Patient eine Hepatomegalie und echoreiches Parenchym aufwies. Die Gallenwege waren in dieser Phase bei allen Patienten unauffällig. Nach 12-24 Monaten zeigten sich sonographisch Zeichen des zirrhotischen Leberumbaus bei weiterhin als unauffällig beschriebenen Gallenwegen.

Das Fehlen von ausgeprägten hepatischen Veränderungen in der Sonographie des Abdomens in unserem Kollektiv (bei nur 2 von 43 Patienten wurde eine Leberzirrhose beschrieben) kam möglicherweise durch die frühe Diagnosestellung zustande (der Median in unserem Kollektiv lag bei 2,5 Monaten vom Ereignis bis zur Diagnose). 
Die Auswertung der computertomographischen Befunde in unserem Kollektiv ergab keine spezifischen Veränderungen. So zeigten 13 von 30 Patienten (43,3\%) eine Erweiterung der intrahepatischen Gallenwege und 7 von 30 Patienten (23,3\%) eine Erweiterung der extrahepatischen Gallenwege. Parenchymunregelmäßigkeiten der Leber wurden bei 2 von 30 Patienten beschrieben. Bei einem Patienten wurden Leberabszesse dokumentiert.

Nur wenige Autoren berichten in einzelnen Fallberichten über CT-Befunde bei Patienten mit ischämischer Cholangiopathie und biliären Casts. So zeigte die CT-Bildgebung bei Katsinelos et al. (2008) eine Gallengangserweiterung ohne sichtbare Läsionen, während Kwon et al. (2012) über multifokale Strikturen und Unregelmäßigkeiten der intrahepatischen Gallenwege bereits nach zwei Wochen bei einem Patienten berichteten.

Die Magnetresonanz-Cholangiopankreatikographie (MRCP) hat einen wichtigen Stellenwert in der nicht invasiven Diagnostik von Erkrankungen der Gallenwege und des Pankreasganges. Bei unserem Kollektiv ist vor Diagnosestellung die MRT der Leber mit MRCP bei einem Patienten durchgeführt worden. Der erhobene Befund bezüglich der Gallenwege war unauffällig. Die durchgeführte ERC-Untersuchung ergab jedoch eine ischämische Cholangiopathie mit Castbildung. Ben-Ari et al. (2015) konnten drei von insgesamt vier Patienten mit ischämischer Cholangiopathie und sekundär sklerosierender Cholangitis mittels MRCP diagnostizieren. Die Befunde in der MRCP zeigten intrahepatisch unregelmäßige Gallenwege mit Strikturen und Dilatationen. Ähnliche Befunde werden in der MRCP auch bei der primär sklerosierenden Cholangitis (PSC) erhoben. Beim Patientengut von Benninger et al. (2005) zeigte die MRT/MRCP in der früheren Phase der Erkrankung (1-2 Monate) einen unauffälligen Befund, während eine MRT/MRCP bei zwei weiteren Patienten (durchgeführt nach 12-24 Monaten) bei einem der Patienten Dilatationen der intrahepatischen Gallenwege sowie Atrophie des linken Leberlappens zeigte, während bei dem anderen Patienten ein zirrhotischer Umbau zu sehen war.

Ergänzend sei vermerkt, dass diese Untersuchungsmethode einen größeren Aufwand erfordert, insbesondere bei beatmeten, kreislaufinstabilen Patienten. Aufgrund von Atemartefakten kann die Aussagekraft deutlich eingeschränkt sein. Hinzu kommt, dass Patienten mit Schrittmachern und künstlichen Herzklappen für diese Untersuchungsmethode nicht in Frage kommen. 


\subsubsection{Histologische Diagnostik}

Bei unserem Patientenkollektiv war eine Leberbiopsie vor Sicherung der Diagnose bei 6 von 51 Patienten $(11,7 \%)$ durchgeführt worden. Dabei wurde in 5 Fällen eine portale Entzündungsreaktion und in 2 Fällen jeweils eine kanalikuläre Cholestase und eine Cholangitis beschrieben. 3 der 6 Befunde beschrieben dabei Veränderungen, die mit einer medikamentös-toxischen Genese vereinbar waren. Nur bei 2 der 6 Patienten war der histopathologische Befund mit einer ischämischen Cholangiopathie vereinbar.

In den frühen Phasen der ischämischen Cholangiopathie mit sekundär sklerosierender Cholangitis (erste Wochen) wurde in der Literatur über eine leichte portale Entzündung mit Infiltration von Lymphozyten und neutrophilen Granulozyten in der Leberbiopsie berichtet, während in den späteren Phasen (nach ca. drei bis vier Monaten) Gallengangsproliferationen, portale und periduktale Fibrose sowie kanalikuläre Cholestase als pathologische Veränderungen vorherrschten (Gelbmann et al. 2007; Esposito et al. 2008). In später durchgeführten Biopsien (nach über einem Jahr ab Diagnosestellung) konnte in den progredienten Fällen ein kompletter zirrhotischer Umbau des Leberparenchyms festgestellt werden (Benninger et al. 2005). Bei Ben-Ari et al. (2015) wurden drei der insgesamt vier Patienten mittels MRCP und Leberbiopsie diagnostiziert. In einer frühen Phase (innerhalb von 3 Monaten) zeigte sich ein portales Ödem mit Neutrophilen-Infiltration. Nach ca. acht Monaten zeigte sich histopathologisch eine frühe Fibrose mit Cholestase und Gallenwegsproliferation sowie biliären Nekrosen. Nach 12 Monaten war bereits ein inkompletter zirrhotischer Umbau festzustellen.

In unserem Kollektiv war die späteste Biopsie vor Diagnosestellung 10 Monate nach dem Intensivaufenthalt durchgeführt worden. In den histologischen Befundergebnissen waren fibrotische Veränderungen, jedoch ohne zirrhotischen Umbau, beschrieben.

\subsection{Endoskopische Verfahren und Therapie}

In unserem untersuchten Patientenkollektiv nimmt die Endosonographie (EUS) des oberen Gastrointesinaltraktes eine untergeordnete Rolle für die Diagnostik einer ischämischen Cholangiopathie ein. Sie kam als diagnostisches Mittel im Rahmen der Abklärung der Cholestase bei fünf Patienten (9,8\% des Gesamtkollektivs) zum Einsatz. Bei 2 der 5 Patienten wurde eine Choledocholithiasis beschrieben. Bei insgesamt 3 Patienten eine extrahepatische Cholestase. Zur Diagnostik einer PSC wird die EUS als wertvolles Verfahren zur Beurteilung 
der extrahepatischen Gallenwege bei gleichzeitig mangelhafter Beurteilbarkeit der intrahepatischen Gallenwege beschrieben (Lutz et al. 2012).

Zur Diagnose der ischämischen Cholangiopathie, die sich primär an den intrahepatischen Gallenwegen abspielt, scheint ihre Aussagekraft beschränkt. Bei keinem unserer Patienten war die EUS an der Diagnosestellung beteiligt. In der Literatur gibt es nach unserer Kenntnis bisher keine Daten zur Wertigkeit der EUS in der Diagnostik einer ischämischen Cholangiopathie bei Intensivpatienten.

Die retrospektive Analyse des vorliegenden Krankenkollektivs mit Entwicklung einer ischämischen Cholangiopathie ggf. zusammen mit sekundär sklerosierender Cholangiopathie während des Intensivaufenthaltes (sog. secondary sclerosing cholangitis in critically ill patients) zeigt, dass die ERC die diagnostische Untersuchung der Wahl darstellt (bei 42 der 51 in der UMG primär diagnostizierten Patienten erfolgte die Diagnose mittels ERC). Auch in der publizierten Literatur wird die ERC als Standard für die Diagnose dieser Erkrankung angesehen (Engler et al. 2003; Benninger et al. 2005; Jaeger et al. 2006; Gelbmann et al. 2007; Voigtländer et al. 2012; Kirchner und Rümmele 2015)

Hier zeigt sich jedoch auch eine gewisse Problematik der Abwägung zum Einsatz der ERC, was möglicherweise bis zum heutigen Tag mit der „hohen Dunkelziffer““ (Albert et al. 2015) an nicht erkannter ischämischer Cholangiopathie bei Intensivpatienten zu tun hat.

In der Regel hat dieses intensivmedizinische Krankengut zahlreiche, teils lebensbedrohliche Komplikationen hinter sich oder leidet noch darunter. Zudem wurden in der Regel zahlreiche Maßnahmen durchgeführt um die zunehmende Cholestase abzuklären ( laborchemische und bakteriologisch/serologische Untersuchungen, Absetzen verschiedenster nicht lebensnotwendiger Medikamente, Umsetzung von Medikamenten, multiple nicht invasive bildgebende Verfahren). Bei Patienten mit Entwicklung einer ischämischen Cholangiopathie waren die meisten dieser Maßnahmen nur begrenzt erfolgreich (teilweise nur kurzfristige Verbesserung der Cholestasewerte).

Die Zurückhaltung bei Patienten, Ärzten, Betreuern, Angehörigen zur Durchführung einer diagnostischen Maßnahme (ERC), ggf. auch in Verbindung mit therapeutischen Interventionen (Cast-Entfernung, endoskopische Behandlung von Strikturen, Papillotomie, etc.), die jedoch auch mit Komplikationen, u.a. ERCP-induzierter Pankreatitis (Lee und Park, 2014) verbunden sein kann, erscheint verständlich. Dies stellt möglicherweise auch einen Grund dar, dass in vielen Kliniken dieses Krankheitsbild nicht frühzeitig erkannt wird. Die Zusammenfassung der meisten publizierten Fälle mit 88 Patienten (Lin et al. 2014) unterstützt diese Aussage. Deshalb ist es dringend notwendig (und die Analyse unseres 
Krankengutes soll dazu beitragen), das Bild der ischämischen Cholangiopathie besonders Intensivmedizinern bekannt zu machen, damit eine frühzeitige Diagnose erfolgen kann.

In unserem Kollektiv lag der Median des zeitlichen Abstandes vom Initialereignis (das im Regelfall auch dem Beginn des Intensivaufenthaltes entsprach) bis zur Diagnosestellung mittels ERC bei 2,5 Monaten. Der längste ermittelte Abstand zwischen Ereignis und Diagnose mittels ERC betrug 42 Monate. Bei 33 von 42 (primär in der UMG diagnostizierten) Patienten (78,5\%) war die Diagnose bereits bei der ersten ERC Untersuchung gestellt worden. Unterschiedliche Zeitpunkte der Diagnosestellung werden auch in der Literatur berichtet. So betrug der Zeitraum vom Anstieg der Cholestasewerte bis zur Diagnose des Krankheitsbildes 4-233 Tage (Gelbmann et al. 2007), 1-7 Monate (Kulaksiz et al. 2008) sowie 2-27 Monate (Benninger et al. 2005).

Die Bildung von Casts, d. h. Ausgüssen im Gallenwegssystem wurde nach unserer Kenntnis erstmalig von Waldram et al. (1975) bei Patienten nach Lebertransplantation beschrieben. Auch in heutiger Zeit stellt die Bildung von Casts, besonders intrahepatisch eine bekannte Komplikation im Rahmen einer ischämischen Cholangiopathie bei lebertransplantierten Patienten dar (Yang et al. 2013). Bei nicht-lebertransplantierten Patienten wird der Nachweis von Casts als sehr selten angesehen (Parry und Muiesan, 2003).

Mit Publikationen über das Krankheitsbild einer ischämischen Cholangiopathie, ggf. mit sekundär sklerosierender Cholangitis bei Intensivpatienten (secondary sclerosing cholangitis in critically ill patients) hat jedoch auch der Nachweis von Casts besonders in den intrahepatischen Gallenwegen (über extrahepatische Casts wurde auch berichtet) zugenommen (Byrne et al. 2003; Gelbmann et al. 2007; Gleeson et al. 2008; Hosoi et et al. 2010; Clermonts und van Dam 2014; Lin et al. 2014; Ben-Ari et al. 2015). In unserem Kollektiv der Patienten mit ischämischer Cholangiopathie nach Intensivaufenthalt wurden bei 42 von 51 Patienten $(82,3 \%)$ Casts in der ERC-Untersuchung beschrieben. Meist gingen sie mit Gallenwegsrarefizierungen, Gallenwegsdilatationen oder Gallenwegsstrikturen einher.

Es erscheint somit, dass die Bildung/Nachweis von Casts bei nicht-lebertransplantierten Patienten nahezu pathognomonisch ist bei Intensivpatienten mit ischämischer Cholangiopathie. Um diesen Eindruck zu objektivieren haben wir im Oktober 2015 bei Pubmed (http://www.ncbi.nlm.nih.gov/pubmed) eine Recherche mit folgenden Termini durchgeführt: biliary casts and primary sclerosing cholangitis, PSC, autoimmune Cholangitis, IgG-Cholangitis, eosinophilic cholangitis, biliary strictures, (secondary) sclerosing cholangitis, ischemic cholangitis, cholangitis, vanishing bile duct syndrome, ductopenia, 
portal hypertensive cholangiopathy. Es zeigte sich, dass Casts nur in Publikationen beschrieben wurden, die über ischämische Cholangiopathie oder secondary sclerosing cholangitis in critically ill patients, berichteten.

Casts, die meistens intrahepatisch lokalisiert sind, aber auch intra- und extrahepatisch oder nur extrahepatisch auftreten können (Gleeson et al. 2008; Hosoi et al. 2010), sind von ihrer Beschaffenheit her relativ hart und derb, von dunkler bis dunkel-grüner Farbe, häufig bei der Extraktion glänzend (Yang et al. 2013).

Die nähere Analyse von Casts zeigte, dass sie aus Bilirubin, zerstörter Gallengangsmukosa, übersättigten Cholesterinkristallen, Blutgefäßen, Kollagenfasern, Kristallablagerungen sowie Bakterien (z.B. Escherichia coli mit Glukuronidaseaktivität) bestehen. Entzündungszellen wie auch Pilze wurden in bisher publizierten Daten, in Casts nicht beschrieben (Waldram et al. 1975; Yang et al. 2013).

Die Mechanismen der Cast-Bildung sind bisher nicht vollständig aufgeklärt. Es scheint ein multifaktorieller Prozess vorzuliegen. Folgende Faktoren scheinen eine Rolle zu spielen: Mukosaschädigung, Ischämie der Gallengangsmukosa, Obstruktion der Gallenwege, Sludgebildung, Infektionen, Cholesterinübersättigung, Viskositätsveränderungen der Galle, Veränderungen des Galleflusses oder parenterale Ernährung (Parry und Muiesan 2003; Waldram et al. 1975; Yang et al. 2013; Angelico und Della Guardia 2000).

In vorliegender Arbeit konnte gezeigt werden, dass ein Teil der endoskopisch geborgenen Casts eine Besiedlung mit Bakterien und Pilzen in der histologischen Untersuchung aufwiesen. Mikrobiologische Kulturen konnten diese Ergebnisse bestätigen. Dieser kombiniert Befall aus bakterieller Besiedlung und Pilzbefall der Gallenwege, die durch Casts teilweise massiv obstruiert sind, hat erhebliche therapeutische Konsequenzen. Diese Befunde der vorliegenden Arbeit unterstreichen die Notwendigkeit einer direkten endoskopischen Gewinnung von Gallesekret/Casts für mikrobiologische Untersuchungen (inclusive Testung auf Antibiotika/Antimykotika Resistenzen) zwecks einer frühzeitigen und zielgerichteten antibakteriellen/antimykotischen Therapie und korrelieren auch mit der Beobachtung von Voigtländer et al. (2015), dass bei Intensivpatienten mit ischämischer Cholangiopathie und sekundär sklerosierender Cholangitis ein direkte Gallegewinnung mit Erregernachweis wahrscheinlich die geeignetste Methode zur Therapieoptimierung ist. 


\section{Zusammenfassung}

Die ischämische Cholangiopathie ist eine relativ neue Erkrankung, die bei intensivmedizinisch behandelten Patienten mit kompliziertem Krankheitsverlauf auftreten kann. Sie wird deshalb auch als secondary sclerosing cholangitis in critically ill patients bezeichnet. Vorherrschend ist eine Cholestase, ggf. mit Ikterus. Die Erkrankung kann rasch progredient verlaufen und zur sekundär biliären Leberzirrhose mit Leberversagen führen. Die Diagnostik der ischämischen Cholangiopathie erfolgt mittels endoskopisch retrograder Cholangiographie (ERC).

51 Patienten (31 Männer, 20 Frauen) mit durch ERC gesicherter Diagnose einer ischämischen Cholangiopathie, die in der Universitätsmedizin Göttingen (UMG) behandelt wurden, wurden retrospektiv (04/2007 - 05/2015) analysiert. Bei diesem Kollektiv wurde bei 42 von 51 Patienten die Diagnose initial in der UMG gestellt. Lebertransplantierte Patienten waren in dem Kollektiv nicht vorhanden.

Das mittlere Alter der Patienten betrug 59,6 \pm 14,2 Jahre. An Vorerkrankungen waren Erkrankungen des kardiovaskulären Systems sowie endokrine und Stoffwechselerkrankungen vorherrschend. Nur ein Patient wies eine Lebervorerkrankung auf (Leberzirrhose), die übrigen Patienten hatten keine Hinweise auf Erkrankungen der Leber oder der Gallenwege.

Als initiales Ereignis, das zur intensivmedizinischen Behandlung führte, waren intrakranielle Blutungen vorherrschend (24/51 Patienten). Der intensivmedizinische Aufenthalt betrug im Median 32 Tage (Range: 13 - 145 Tage). Die Dauer der invasiven Beatmung lag im Median bei 22 Tagen (Range: 9 - 72 Tage). Invasive Maßnahmen während des Intensivaufenthaltes beinhalteten Tracheotomien (72\%), Einlage von intrazerebralen Drainagen (45\%) sowie neurochirurgische Operationen (34\%).

Medikamentöse Therapien während des Intensivaufenthaltes bestanden aus Ketamin und Midazolam (60\%), Opioiden (40\%) sowie Propofol (21\%). Als Antibiotika wurden vorwiegend Penicilline (69 \%), gefolgt von Carbapenemen (57 \%) verabreicht. Katecholamine erhielten 23/42 Patienten (57\%).

Über einen Zeitraum von sechs Wochen (Ereignistag 1 bis Tag 42) wurden verschiedene Laborparameter (AST, ALT, Quick-Wert, J-GT, AP, Gesamtbilirubin, Hämoglobin (Hb), Leukozyten, Kreatinin) analysiert. Bei Patienten mit ischämischer Cholangiopathie zeigte sich vor Diagnosestellung ein ausgeprägter Anstieg der Cholestaseparameter ( $\mathrm{z}-\mathrm{GT}$ 19-Fach, 
Bilirubin 12-Fach, AP 6-Fach erhöht). Die Transaminasen AST und ALT zeigten hingegen nur 3- bis 5-Fache Anstiege im Beobachtungszeitraum. Der Quick-Wert, Kreatinin sowie Leukozyten blieben nahezu stabil.

Alle Patienten wiesen ab der zweiten Woche des Intensivaufenthaltes eine Anämie auf. Die $\mathrm{Hb}-W e r t e$ fluktuierten zwischen $8-9 \mathrm{~g} / \mathrm{dl}$. Bei normalem arteriellem $\mathrm{pO}_{2}$-Wert entspricht diese Konstellation einer anämischen Hypoxie. Die Parameter der arteriellen Blutgasanalyse blieben über vier Wochen stabil und lagen im Normbereich.

Mikrobiologische Untersuchungen in verschiedenen Kompartimenten (vor Diagnosestellung) zeigten, dass Staphylococcus epidermidis in Blutkulturen vorherrschend war (9/21 Patienten), bei 6/9 Patienten lag ein Oxacillin-resistenter Keim vor. Escherichia coli war bei 6/21 Patienten nachweisbar. Im Galleaspirat (15/51 Patienten) war Enterococcus faecium vorherrschend (7/15 Patienten). Eine bakterielle wie auch mykologische Koinfektion mit Candida albicans konnte bei 6/15 Patienten kulturell nachgewiesen werden. Diese Koinfektion konnte auch in geborgenen Casts histopathologisch dargestellt werden.

Die bildgebende Diagnostik (Abdominalsonographie inklusive Duplexsonographie, Computertomographie, Magnetresonanztomographie mit Darstellung der Gallenwege, Endosonographie) war nicht wegweisend für die Diagnosestellung einer ischämischen Cholangiopathie.

Die vorliegende retrospektive Analyse von Patienten mit einem komplizierten intensivmedizinischen Verlauf mit Entwicklung einer Cholestase/Ikterus bestätigt, dass die ERC den Goldstandard zur Diagnosestellung dieser Erkrankung darstellt. Bei initial in der UMG diagnostizierten Patienten erfolgte die Diagnosestellung mittels ERC im Median 2,5 Monate nach dem Ereignis. Bei 42/51 Patienten konnten Casts festgestellt werden, die somit nahezu pathognomonisch für diese Erkrankung anzusehen sind. Durch Entfernung der Casts via Papillotomie ist die ERC auch als Goldstandard zur Verbesserung des Galleabflusses anzusehen.

In künftigen prospektiven Untersuchungen sollte geklärt werden, möglichst unter Bereitstellung einer Kontrollgruppe, welchen Einfluss die Anämie (,anämische Hypoxie“), die bei all unseren Patienten nachweisbar war, auf die Entstehung einer ischämischen Cholangiopathie bei schwerkranken Intensivpatienten hat. 


\section{$7 \quad$ Literaturverzeichnis}

Abdalian R, Heathcote EJ (2006): Sclerosing cholangitis: A focus on secondary causes. Hepatology 44, 1063-1074

Albert J, Aabakken L, Barthet M, Le Moine O, Seewald S: Endoscopic Retrograde Cholangio-Pankreaticography (ERCP) - Current Practice and Future Perspectives. 1. Auflage; UNI-MED Verlag AG, Bremen 2015, 105-108

Algahtani SA, Kleiner DE, Ghabril M, Gu J, Hoofnagle JH, Rockey DC, Drug-induced Liver Injury Network (DILIN) Study Investigators (2015): Identification and Characterisation of cefazolin-Induced Liver Injury. Clin Gastroenterol Hepatol 13(7), 1328-1336

Andersen LW, Mackenhauer J, Roberts JC, Berg KM, Cocchi MN, Donnino MW (2013): Etiology and therapeutic approach to elevated lactate levels. Mayo Clin Proc $\underline{88}$ (10), 11271140

Angelico M, Della Guardia P (2000): Review article: hepatobiliary complications associated with total parenteral nutrition. Aliment Pharmacol Ther 14(2), 54-57

Barton P, Maier A, Steininger R, Mühlbacher F, Lechner G (1995): Biliary sludge after liver transplantation: 1. Imaging findings and efficiacy of various procedures; 2. Treatment with interventional techniques versus surgery and/or oral chemolysis. AJR 164(4), 859-869

Batts KP (1998): Ischemic cholangitis.

Mayo Clin Proc 73(4), 380-385

Belardo DO, Melo JF, Bonfim AV, Teixeira AA, Teixeira RA, Duarte AL (2013):

Acute cholestatic hepatitis caused by amoxicillin/clavulanate. World J Gastroenterol 19(46), 8789-8792 
Ben-Ari Z, Levingston D, Weitzman E, Haviv-Yadid Y, Cohen-Ezra O, Weiss P, Zuckerman E, Inbar Y, Amitai M, Rimon U, Goder M, Haik J (2015): Secondary sclerosing cholangitis following major burn. Ann Hepatol 14(5), 695-701

Benninger J, Grobholz R, Oeztuerk Y, Antoni CH, Hahn EG, Singer MV, Strauss R (2005): Sclerosing cholangitis following severe trauma: Description of a remarkable disease entity with emphasis on possible pathophysiologic mechanism. World J Gastroenterol 11(27), 41994205

Byrne MF, Chong HI, O`Donovan D, Sheehan KM, LeaderMB, Kay E, McCormick PA, Broe P, Murray FE (2003): Idiopathic cholangiopathy in a biliary cast syndrome necessitating liver transplantation following head trauma. Eur J Gastroenterol Hepatol 15(4), 415-417

Chan EY, Olson LC, Kisthard JA, Perkins DJ, Bakthavatsalam R, Halldorson JB, Reyes JD, Larson AM, Levy AE (2008): Ischemic cholangiopathy following liver transplantation from donation after cardiac death donors. Liver Transpl 14(5), 604-610

Chand N, Saynal AJ (2006): Sepsis-induced cholestasis. Hepatology, 45, 230-241

Chapman R, Fevery J, Kalloo A, Nagorney DM, Boberg KM, Schneider B, Gores G (2009): Diagnosis and management of primary sclerosing cholangitis. Hepatology $\underline{51}(2), 660-678$

Clermonts SH, van Dam RM (2014): Obstructive putty-like cast of the biliary tree. Hepatobiliary Surg Nutr $\underline{3}(1), 47-49$

Deltenre P, Valla DC (2006): Ischemic cholangiopathy. J Hepatol 444(4), 806-817

Desormais I, Aboyans V, Bura A, Constans J, Cambou JP, Messas E, Labrunie A, Lacrois P (2014): Anemia, an independent predictive factor for amputation and mortality in patients hospitalized for peripheral artery disease. Eur J Vasc Endovasc Surg 48 (2), 202-207

Engler S, Elsing C, Flechtenmacher C, Theilmann L, Stremmel W, Stiehl A (2003): Progressive sclerosing cholangitis after septic shock: a new variant of vanishing bile duct

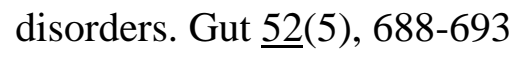


Esposito I, Kubisova A, Stiehl A, Kulaksiz H, Schirmacher P (2008): Secondary sclerosing cholangitis after intensive care unit treatment: clue to the histopathological differential diagnosis. Virchow Arch 453(4), 339-345

Ganong, WF: Review of Medical Physiology. 22. Auflage; Lange Medical Books/McGrawHill, New York 2005, 681-697

Gelbmann C, Rümmele P, Wimmer M, Hofstädter F, Göhlmann B, Endlicher E, Kullmann F, Langgartner J, Schölmerich J (2007): Ischemic-like cholangiopathy with secondary sclerosing cholangitis in critically ill patients. Am J Gastroenterol 102(6), 1221-1229

Gleeson FC, Czaja AJ, Baron TH (2008): Successful endoscopic management of biliary cast syndrome in nonliver transplant patients. J Clin Gastroenterol 42(6), 752-755

Go AS, Yang J, Ackerson LM, Lepper K, Robbins S, Massie BM, Shlipak MG (2006): Hemoglobin level, chronic kidney disease, and the risk of death and hospitalization in adults with chronic heart failure: the Anemia in Chronic Heart Failure: Outcomes and Resource Utilization (ANCHOR) Study. Circulation 113(23), 2713-23

Gor NV, Levy RM, Ahn J, Kogan D, Dodson SF, Cohen SM (2008): Biliary cast syndrome post-liver transplantation: risk factors and outcome. Liver Transpl 14(10), 1466-1472

Gossard AA; Angulo P, Lindor KD (2005): Secondary sclerosing cholangitis: A comparison to Primary Sclerosing Cholangitis. Am J Gastroenterol 100(6), 1330-1333

Hare GM, Freedmann J, Mazer CD (2013): Rewiew article: risks of anemia and related management strategies: can perioperative blood management improve patient safety? Can J Anaesth $\underline{60}(2), 168-175$

Heidenhain C, Pratschke J, Puhl G, Neumann U, Pascher A, Veltzke-Schlieker W, Neuhaus P (2010): Incidence of and risk factors for ischemic-type biliary lesions following orthotopic liver transplantation. Transpl Int 23(1), 14-22 
Hiltebrand LB, Krejci V, Sigurdsson GH (2004): Effects of dopamine, dobutamine and dopexamine on microcirculatory blood flow in the gastrointestinal tract during sepsis and anesthesia. Anesthesiology 100(5), 1188-1997

Hohn D, Melnick J, Stagg R, Altman D, Friedman M, Ignoffo R, Ferrell L, Lewis B (1985): Biliary sclerosis in patients receiving hepatic arterial infusions of floxuridine. J Clin Oncol $\underline{3}(1), 98-102$

Hosoi M, NannyaY, Sasaki T, Suzuki HI, Ueda K, Tsujino T, Takahashi T, Koike K, Kurokawa M (2010): Biliary cast syndrome and benign bilary stricture as complications of

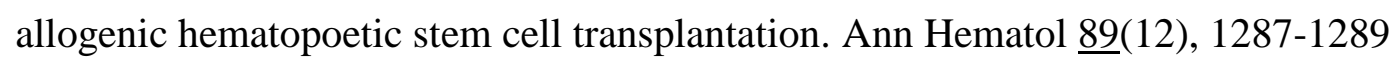

Jaeger C, Mayer G, Heinrich R, Gossner L, Rabenstein T, May A, Guenter E, Ell C (2006): Secondary sclerosing cholangitis after long term treatment in an intensive care unit: Clinical presentation, endoscopic findings, treatment, and follow up. Endoscopy $\underline{38}$ (7), 330-334

Katsinelos P, Kountouras J, Chatzimavroudis G, Zavros C, Pilpillidis J, Paroutoglou G (2008): Combined endoscopic and ursodeoxycholic acid treatment of biliary cast syndrome in a non-transplant patient. World J Gastroenterol 14(33), 5223-5225

Keymling M, Kohler BM, Lübke HJ: Das ERCP-Buch. Georg Thieme Verlag, Stuttgart 2013, $185-187$

Kirchner GI, Rümmele P (2015): Update on sclerosing cholangitis in critically ill patients. Viszeralmedizin $\underline{31}, 178-184$

Krejci V, Hiltebrand LB, Sigurdsson GD (2006): Effects of epinephrine, norepinephrine and phenylepinephrine on microcirculatory blood flow in the gastrointestinal tract in sepsis. Crit Care Med. 34(5), 1456-63

Kulaksiz H, Heuberger D, Engler S, Stiehl A (2008): Poor outcome in progressive sclerosing cholangitis after septic shock. Endoscopy $\underline{40}(3), 214-218$ 
Kwon ON, Cho SH, Park CK, Mun SH (2012): Biliary cast formation with sclerosing cholangitis in critically ill patients: case report and literature review. Korean J Radiol 13(3), $358-362$

Lee CJ, Subeg YM, Lee RP, Wu WT, HSU BG (2008): Low-dose propofol ameliorates haemorrhagic shock-induced organ damage in conscious rats. Clin Exp Pharmacol Physiol $\underline{35}(7), 766-74$

Lee TH, Park H (2014): Endoscopic prevention of post-endoscopic retrograde cholangiopancreaticography pancreatitis. World J Gastroenterol 20(44), 16582-95

Leonhardt S, Veltzke-Schlieker W, Adler A, Schott E, Hetzer R, Schaffartzik W, Tryba M, Neuhaus P, Seehofer D (2015): Trigger mechanism of secondary sclerosing cholangitis in critically ill patients. Crit Care $\underline{19}(1), 131$

Li S, Stratta RJ, Langnas AN, Wood RP, Marujo W, Schaw BW Jr (1992): Diffuse biliary tract injury after orthotopic liver transplantation. Am J Surg 164(5), 536-540

Lin T, Qu K, Xu X, Tian M, Gao J, Zhang C, Di Y, Zhang Y, Liu C (2014): Sclerosing cholangitis in critically ill patients: an important and easily ignored problem based on a German experience. Front Med $\underline{8}(1), 118-126$

Lison S, Weiss G, Spannagl M, Heindl B (2011): Postoperative changes in procoagulant factors after major surgery. Blood Coagul Fibrinolysis 22(3), 190-196

Lo RSC, Krischnamoorthy R, Freemann JG, Austin AS (2011): Cholestasis and biliary dilatation associated with chronic ketamine abuse: a case series. Singapore Med J $\underline{52}$ (3), e52e55

Lui KL, Lee WK, Li MKK (2014): Ketamin-induced cholangiopathy. Hong Kong Med J 20, 78.e1-2 
Lutz HH, Wasmuth HE, Streetz K, Tacke F, Koch A, Luedde T, Trautwein C, Tischendorf JJW (2012): Endoscopic ultrasound as an early diagnostic tool for primary sclerosing cholangitis: a prospective pilot study. Endoscopy 44(10), 934-939

MacIntyre NR (2014): Tissue hypoxia: implications for the respiratory clinician. Respir Care $\underline{59}(10), 1590-1600$

Martineau G, Porter KA, Gorman J, Launois B, Schroter GT, Palmer W, Putnam CW, Groth CG, Halgrimson CG, Penn I, Starzl TE (1972): Delayed biliary duct obstruction after orthotopic liver transplantation. Surgery $\underline{72}$ (4), 604-610

Meier J, Müller MM, Lauscher P, Sireis W, Seifried E, Acharowski K (2012): Perioperative Red Blood Cell Transfusion: Harmful or Beneficial to the Patient? Transfus Med Hemother $\underline{39}(2), 98-103$

Mourad MM, Algarni A, Liossis C, Bramhall SR (2014): Aetiology and risk factors of ischaemic cholangiopathy after liver transplantation.World J Gastroenterol 20(20), 6159-6169

Parry SD, Muiesan P (2003): Cholangiopathy and the biliary cast syndrome. Eur J Gastroenterol Hepatol 15(4), 341-342

Quattropani C, Schneider M, Helbing A, Zimmermann A, Krähnenbühl S (2001): Cholangiopathy after short-term administration of piperacillin and imipenem/cilastin. Liver $\underline{21}(3), 213-216$

Ruemmele P, Hofstaedter F, Gelbmann CM (2009): Secondary sclerosing cholangitis. Nat Rev Gastroenterol Hepatol $\underline{6}(5), 287-295$

Ryley NG, Fleming KA, Chapman RW (1995): Focal destructive cholangiopathy associated with amoxycillin/clavulanic acid (Augmentin). J Hepatol 233(3), 278-282

Sanchez-Urdazpal L, Gores GJ, Ward EM, Maus TP, Buckel EG, Steer JL, Wiesner RH, Krom RA (1993): Diagnostic features and clinical outcome of ischemic-type biliary complications after liver transplantation. Hepatology 17(4), 605-609 
Scheppach W, Druge G, Wittenberg G, Mueller JG, Gassel AM, Gassel HJ, Richter F (2001): Sclerosing cholangitis and liver cirrhosis after extrabiliary infections: Report on three cases. Crit Care Med 29(2), 438-441

Shah JN, Haigh WG, Sum PD, Lee P, Lucey MR, Bresinger CM, Kochman ML, Long WB, Olthoff K, Shaked A, Gingsber GG (2003): Biliary casts after orthotopic liver transplantation: Clinical factors, treatment, biochemical analysis. Am J Gastroenterol 98(8), 1861-1867

Siddique A, Kowdley KV (2012): Approach to a patient with elevated serum alkaline phosphatase. Clin Liver Dis $\underline{16}(2), 199-229$

Soni N, Fawcett WJ, Halliday FC (1993): Beyond the lung: oxygen delivery and tissue oxygenation. Anaesthesia $\underline{48}(8), 704-711$

Tavakol K, Ghahramanpoori B, Fararouei M (2013): Prediction of arterial blood pH and Partial Pressure of Carbon dioxide from Venous Blood Samples in Patients Receiving Mechanical Ventilation. J Med Signals Sens $\underline{3}(3), 180-184$

Thomas L: Labor und Diagnose, Indikation und Bewertung von Laborbefunden für die medizinische Diagnostik. 8. Auflage; Band 1, TH-Books Verlagsgesellschaft $\mathrm{mbH}$, Frankfurt/Main 2012, 336-337

Thomas RS, Voigtlaender T, Lankisch TO (2014): Sekundär sklerosierende Cholangitis bei und nach intensivmedizinischer Behandlung und in der neurologischen Frührehabilitation. Akt Neurol 41, 140-145

Turkish A, Luo JJ, Leftowitch JH (2013): Ketamine abuse, biliary tract, and secondary sclerosing cholangitis. Hepatology $\underline{58}(2), 825-827$

Vitellas KM, Keogan MT, Freed KS, Enns RA, Spritzer CE, Baillie JM, Nelson RC (2000): Radiologic manifestations of sclerosing cholangitis with emphasis on MR Cholangiopancreaticography. Radiographics 20(4), 959-975 
Voigtländer T, Negm AA, Schneider AS, Strassburg CP, Manns MP, Wedemeyer J, Lankisch TO (2012): Secondary sclerosing cholangitis in critically ill patients: Model of End-StageLiver Disease score and renal function predict outcome. Endoscopy $\underline{44(11), 1055-1058}$

Voigtländer T, Negm AA, Strassburg CP, Lehner F, Manns M, Lankisch T (2013): Biliary cast syndrome post-liver transplantation: risk factors and outcome. Liver Int 33(8), 1287-1292

Voigtländer T, Leuchs E, Vonberg RP, Solbach P, Manns MP, Suerbaum S, Lankisch TO (2015): Microbiological analysis of bile and its impact in critically ill patients with secondary sclerosing cholangitis. J Infect 70(5), 483-490

Waldram R, Williams R, Calne RY (1975): Bile composition and the cast formation after transplantation oft the liver in men. Transplantation 19 (5), 382-387

Weig T, Schubert MI, Gruener N, Dolch ME, Frey L, Miller J, Johnson T, Irlbeck M (2012): Abdominal obesity and prolonged prone positioning increase risk of developing sclerosing cholangitis in critcally ill patients with influenza A-associated ARDS.

Eur J Med Res 17(1), 30

Wong SW, Lee KF, Wng J, Ng WWC, Cheung YS, Lai PBS (2009): Dilated common bile ducts mimicking choledochal cysts in ketamine abusers. Hong Kong Med J $\underline{15}$, 53-56

Yang YL, Zhang C, Lin MJ, Shi LJ, Zhang HW, Li JY, Yu Q (2013): Biliary casts after liver transplantation: morphology and biochemical analysis.

World J Gastroenterol 19(43), 7772-7778 


\section{Danksagung}

Ich danke meinem Doktorvater, Herrn Prof. Dr. med. H. Schwörer, für die Bereitstellung des Themas sowie für die zahlreichen Diskussionen und wertvollen Verbesserungsvorschläge. Meinem Chef Herrn Prof. Dr. med. V. Ellenrieder danke ich für sein stetiges Interesse und seine Unterstützung bei der Durchführung der vorliegenden Arbeit.

Weiterhin möchte ich mich bei Frau Dr. Kitz und Herrn Dr. Bürger für ihre freundliche Hilfe und Unterstuitzung bei der Beurteilung des histopathologischen Bildmaterials, das in dieser Arbeit verwendet wurde, bedanken.

Darüber hinaus danke ich Frau Böning, die mir bei der Recherche der Patientenunterlagen stets hilfreich zur Seite stand, Herrn Dr. Wagner (GIZ-Nord) und Herrn Sobotta, die mich bei den Hürden der Datenauflistung und Auswertung mittels Windows Computerprogrammen unterstützten. 


\section{Lebenslauf}

Am 23.03.1980 wurde ich, Ardian Mekolli, in Prishtina (Kosovo) geboren.

Meine Schulausbildung absolvierte ich von 1986 bis 1994 in der Grund- und Mittelschule und von 1994 bis 1998 am Gymnasium in Prishtina, wo ich 1998 das Abitur ablegte.

Anfang des Jahres 1999 belegte ich nach meinem Umzug nach Deutschland als Vorbereitung für ein Medizinstudium an der Georg-August-Universität in Göttingen einen deutschen Sprachkurs des ASTA Göttingen. Im April 2000 legte ich die Deutsche Sprachprüfung für den Hochschulzugang ausländischer Studienbewerber in Göttingen erfolgreich ab und nahm das Studium der Humanmedizin an der Georg-August-Universität Göttingen auf. Während der Studienzeit ging ich von 2001-2007 einer Nebentätigkeit als studentische Aushilfskraft in der Patientenpflege im Universitätsklinikum Göttingen nach. Nach der vorklinischen Studienphase legte ich im April 2002 die Ärztliche Zwischenprüfung (Physikum) ab. Nach der klinischen Studienperiode absolvierte ich von August 2005 bis Juli 2006 mein Praktisches Jahr. Nach dem Studienabschluss mit dem Staatsexamen im Juni 2007 begann ich meine berufliche Tätigkeit als Assistenzarzt/Weiterbildungsassistenz in der Abteilung für Innere Medizin des Eichsfeld-Klinikums in Bad Heiligenstadt. Anschließend an diese Beschäftigung trat ich im Januar 2009 meine Stelle als Assistenzarzt in der Abteilung für Gastroenterologie und Endokrinologie im Universitätsklinikum Göttingen an. Seit April 2014 bin ich in der Klinik für Gastroenterologie und gastrointestinale Onkologie (Direktor: Prof. Dr. med. V. Ellenrieder) als Assistenzarzt tätig.

Die hier vorliegende Dissertationsschrift habe ich im August 2014 begonnen und im Oktober 2015 abgeschlossen. 